NASA Reference Publication 1051

NBSIR 79-1611

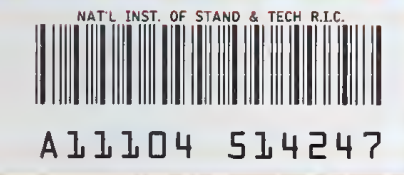

Thermodynamic Properties of Nitrogen Gas Derived From Measurements of Sound Speed

Ben Younglove and R. D. McCarty 

NASA Reference Publication 1051

JUL 201981

NBSIR 79-1611

\section{Thermodynamic Properties of Nitrogen Gas Derived From Measurements of Sound Speed}

Ben Younglove and $\mathbb{R}$. D. McCarty

Thermopbysical Properties Division

National Engineering Laboratory

National Bureau of Standards

Boulder, Colorado

Prepared for NASA Langley Research Center

\section{N/SA}

National Aeronautics

and Space Administration

Scientific and Technical

Information Branch 



\section{SUMMARY}

A virial equation of state for nitrogen has been determined by use of newly measured speed-of-sound data and existing pressure-density-temperature data in a multiproperty fitting technique. The experimental data taken were chosen to optimize the equation of state for a pressure range of 0 to 70 atm and for a temperature $\mathrm{r}$ ange of 60 to $350 \mathrm{~K}$. Comparisons are made for thermodynamic properties calculated both from the new equation and from existing equations of state.

\section{INTRODUCTION}

Over the past several years, there has been renewed interest in gaseous nitrogen in the pressure range of 0 to $10 \mathrm{~atm}$ and the temperature range of 60 to $350 \mathrm{~K}$. This interest has grown out of the development of the cryogenic wind-tunnel concept at the Langley Research Center (LaRC) of the National Aeronautics and Space Administration (NASA). This new wind-tunnel concept uses nitrogen gas at cryogenic temperatures to obtain aerodynamic data at high Reynolds numbers and at transonic Mach numbers. The need for an accurate equation of state became apparent as real-gas effects on flow simulation and datareduction requirements were studied. Although the well-known equations of state (Jacobsen and Beattie-Bridgeman, refs. 1 and 2, respectively) were readily available, discussions between NASA and the National Bureau of Standards (NBS) revealed that very little experimental data were available for gaseous nitrogen at the time of their development to substantiate these equations of state in the pressure range of interest. Because of the lack of experimental data, it was believed that substantial uncertainty could exist in thermodynamic quantities derived from either the Jacobsen or the Beattie-Bridgeman equation. To eliminate this uncertainty and to provide an equation of state for gaseous nitrogen optimized to the more limited pressure and temperature regions used with cryogenic tunnels, NBS has taken sound-speed data (ref. 3) for the conditions of interest and has used a virial equation of state and a multiproperty fitting technique (ref. 4) to arrive at an optimized equation of state.

The authors would like to thank Robert M. Hall and Jerry B. Adcock of NASA-LaRC who contributed significantly to the contents of this report.

\section{SYMBOLS}

Symbols shown below in parentheses are those used in table I.

a (VEL) sound speed, $\mathrm{m} / \mathrm{sec}$

B (BO) second virial coefficient, liters/mol

(B1) first derivative of $B$ 
(B2) second derivative of $B$

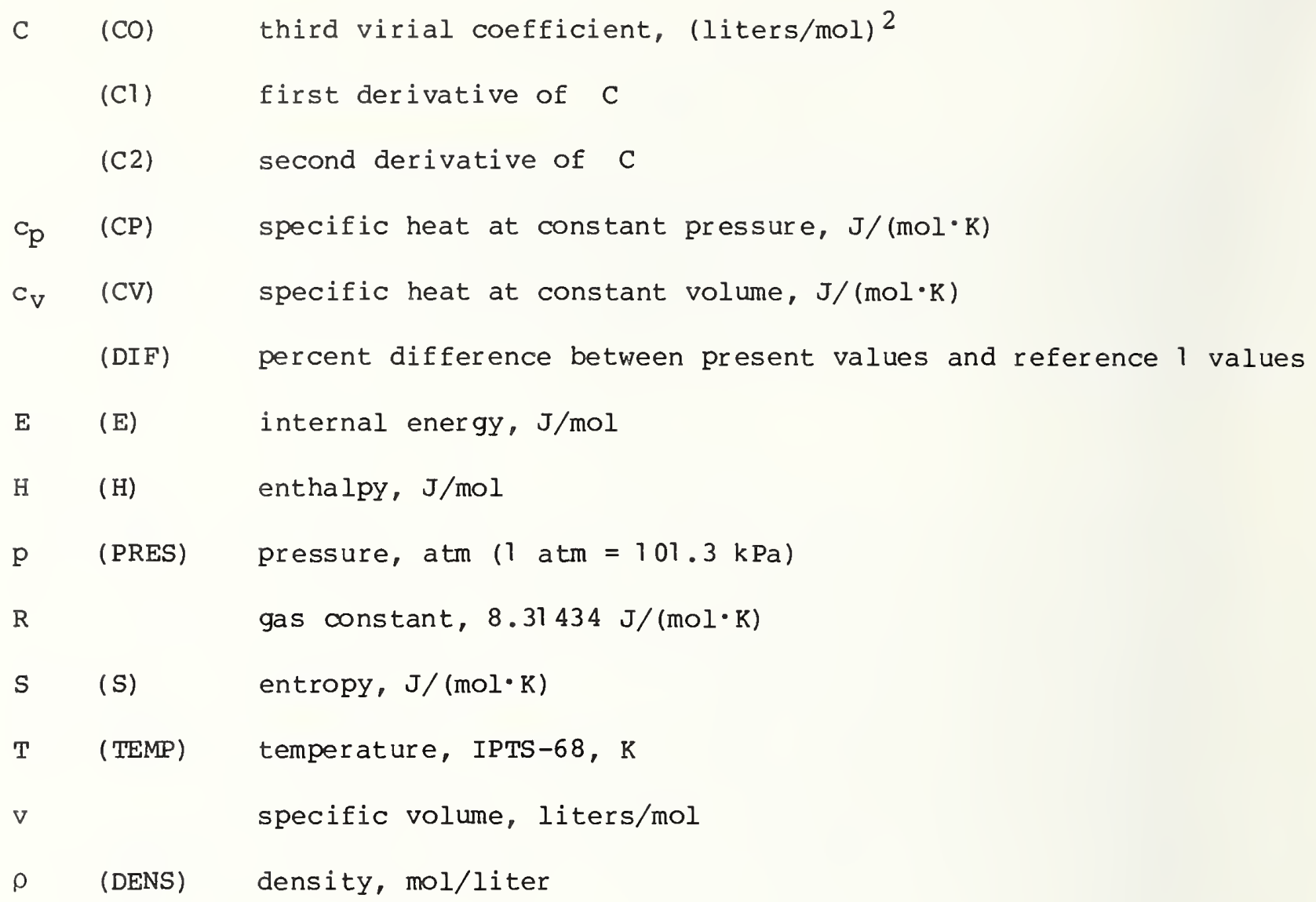

\section{MULTIPROPERTY FITTING}

The use of sound-speed measurements for calculating thermodynamic properties is well established (refs. 5 to 7). The sound speed relates to derivatives of the primary thermodynamic quantities $p, \rho$, and $T$ in a nonlinear fashion. For example,

$$
a^{2}=\left(\frac{\partial p}{\partial \rho}\right)_{S}
$$

or, equivalently,

$$
a^{2}=\left(\frac{c_{p}}{c_{v}}\right)\left(\frac{\partial p}{\partial \rho}\right)_{T}
$$


and

$$
a^{2}=\left(\frac{T}{\rho^{2} c_{V}}\right)\left(\frac{\partial p}{\partial T}\right)_{\rho}^{2}+\left(\frac{\partial p}{\partial \rho}\right)_{T}
$$

It is this nonlinearity which prevents the use of conventional linear leastsquares fitting techniques (ref. 4).

The pressure and the sound speed are computed from the density virial expansion

$$
p=R T \rho\left(1+B \rho+C \rho^{2}\right)
$$

The virial coefficients $B$ and $C$ are in turn represented by an assumed power expansion in temperature as discussed later in the paper. Coefficients from which $B$ and $C$ are computed are found by using multiproperty fitting of two sets of data.

Multiproperty fitting is a technique which allows the use of different kinds of thermodynamic data simultaneously in a least-squares estimation of the adjustable parameters in an equation of state. In the present case this technique allows the simultaneous use of $p-\rho-T$ data and sound-speed data to determine a virial equation of state. The technique is especially appropriate for this particular combination of data forms to determine the second virial coefficient $B$. An additional advantage is obtained in this case because the $B$ in equation (4), which is the density virial expansion, and the $B$ in

$$
\mathrm{pv}=\mathrm{RT}+\mathrm{Bp}
$$

which is the pressure virial expansion, are interchangeable. Therefore, an expression for the sound speed may be derived from equation (5) which contains only the variables $p, a$, and $T$ (i.e., no density). These are the actual measured variables. This expression is then combined with equation (4) by multiproperty fitting to determine $B$ in the density virial expansion. For details of this process, see reference 4.

\section{EXPERIMENTAL METHOD}

The sound speed was measured with a fixed-path-length cavity, or resonator. Electrostatic transducers were used for sound generation and detection. A standing wave is produced by exciting the generating transducer with a variable frequency. The frequency of the oscillator is adjusted for maximum received signal, which occurs at the cavity resonance. At resonance there are an integral number $\mathrm{N}$ of half-wavelengths in the tube of length $\mathrm{L}$, 


$$
\frac{N \lambda}{2}=L
$$

The sound speed is computed from

$$
\mathrm{a}=\mathrm{F} \lambda
$$

Generally, frequencies $F$ were measured corresponding to resonances for $\mathrm{N}=6,8,10,12,14$, or 16 . Three to five resonance frequencies were measured and the experimental sound speed was taken to be the average of the velocities computed from the various frequencies.

The cavity wall is a tube of Invar $67 \mathrm{~mm}$ long and $10 \mathrm{~mm}$ in diameter. The electrostatic transducers are at the ends of the cavity. The inside wall of the tube was ground and polished to allow proper evaluation of the boundary effects. The sound speed inside the tube is slower than it would be in an unbounded medium because of energy losses to the walls and ends via thermal conduction and viscous drag. This difference in sound speed can be computed accurately by using thermodynamic properties such as viscosity, thermal conductivity, heat capacity, and density of the gas medium (ref. 1). For the present experimental method, the size of this correction was generally a few hundredths of a percent in sound speed; however, it increased to about 0.5 percent in the worst case, which occurred at low temperature and high pressure.

The active transducer surface was a thin, flexible plastic film (ref. 8), coated on one side with aluminum of a thickness of approximately $50 \mathrm{~nm}(500 \AA)$. The conducting side was placed on the ends of the tube and stretched tightly so as to produce a flat surface. Immediately on the other side of the coated surface were placed the electrodes, which were copper discs. The signal strength applied to the electrodes was 50 to $100 \mathrm{~V}$ at 1 to $30 \mathrm{kHz}$. The transducers produce sound of measurable intensity over this range of frequencies. Over the frequency variation of the width of a single resonance the sound intensity is essentially constant.

Temperatures were measured on the International Practical Temperature Scale-68 (IPTS-68) with a platinum resistance thermometer calibrated by the NBS Heat Section. Uncertainties in the measured temperature were estimated to be $5 \mathrm{mK}$ at the lower temperatures and approximately $50 \mathrm{mK}$ at $350 \mathrm{~K}$. Pressures were measured with a precision quartz spiral Bourdon tube, calibrated at the National Engineering Laboratory with an air-dead-weight gage. Pressure uncertainties were estimated to be less than 0.013 percent at 15 atm, increasing to 0.3 percent at $0.33 \mathrm{~atm}$. The sample gas was ultrapure carrier type. Analysis of the nitrogen gas by the supplier showed an oxygen concentration of $1 \mathrm{ppm}$ and total hydrocarbon concentration of less than $0.2 \mathrm{ppm}$.

To estimate the uncertainty involved in the overall experimental procedure, sound-speed measurements of helium gas were taken in the apparatus and were 
found to be within 0.05 percent of known values. This value of $0.05-$ percent uncertainty is taken to be the uncertainty in the present nitrogen measurements.

\section{RESULTS}

The density virial coefficients resulting from application of the multiproperty fit to sound-speed and $p-p-T$ data are based on temperature expansions. The form of the second virial coefficient is expressed as

$$
B=\sum B_{j} T^{(3-j) / 2} \quad(j=1,2,3, \ldots, 7)
$$

and the form of the third virial coefficient is expressed as

$$
c=\sum c_{j} T^{(1-j) / 2} \quad(j=1,2,3, \ldots ., 5)
$$

The newly calculated numerical values of $B$ and $C$ are given in the following table:

\begin{tabular}{|c|c|}
\hline $\begin{array}{c}\text { Virial } \\
\text { coefficient }\end{array}$ & $\begin{array}{l}\text { Calculated numerical } \\
\text { values of } \mathrm{B} \text { and } \mathrm{C}\end{array}$ \\
\hline $\mathrm{B}_{1}$ & $0.2920642838 \mathrm{E}-02$ \\
$\mathrm{~B}_{2}$ & $-.2334399080 \mathrm{E}+00$ \\
$\mathrm{~B}_{3}$ & $.7425086518 \mathrm{E}+01$ \\
$\mathrm{~B}_{4}$ & $-.1164300428 \mathrm{E}+03$ \\
$\mathrm{~B}_{5}$ & $.9253305729 \mathrm{E}+03$ \\
$\mathrm{~B}_{6}$ & $-.3351637808 \mathrm{E}+04$ \\
$\mathrm{~B}_{7}$ & $.1992311954 \mathrm{E}+04$ \\
$\mathrm{C}_{1}$ & $.2189970095 \mathrm{E}-01$ \\
$\mathrm{C}_{2}$ & $-.5949349694 \mathrm{E}-01$ \\
$\mathrm{C}_{3}$ & $-.2399796650 \mathrm{E}+02$ \\
$\mathrm{C}_{4}$ & $.4534244413 \mathrm{E}+03$ \\
$\mathrm{C}_{5}$ & $-.2283997281 \mathrm{E}+04$ \\
\hline
\end{tabular}

The values for the thermodynamic properties for nitrogen computed from equations (8) and (9) for $B$ and $C$ are given in table I. Values are computed for isotherms starting at $60 \mathrm{~K}$, increasing at $10-\mathrm{K}$ increments to $110 \mathrm{~K}$ and at $20-\mathrm{K}$ increments from 110 to $350 \mathrm{~K}$. The second and third density virial coefficients with the first and second derivatives of each are shown at the top of each page. For most isotherms the property values are computed at pressures beginning at $0.2 \mathrm{~atm}$ and increasing to $10 \mathrm{~atm}$ in $0.2-\mathrm{atm}$ increments. The values for two-phase conditions are indicated by the notation "2-PHASE BOUNDARY" shown at the right side of the table. Values for the thermodynamic properties are given beyond the two-phase boundary as an indication of the properties for metastable conditions. 
The tabular values of the thermodynamic properties are arranged for comparison of the values computed from the virial coefficients with the existing values from NBS Technical Note 648 (ref. 1). The first column for each case is computed from the virial equation. The numbers of the different columns are presented as percent of deviation from TN 648 values in the graphs in figures 1 to 7. However, above approximately $150 \mathrm{~K}$ the differences are omitted from the graphs because they generally are very close to the values of Technical Note 648 in that region.

The largest deviations (approximately 6 percent) were found for $\mathrm{c}_{\mathrm{p}}$ and $\mathrm{c}_{\mathrm{V}}$ for the values at saturation in figure $\mathrm{l}$ and for $\mathrm{c}_{\mathrm{p}}$ for the $100-\mathrm{K}^{\mathrm{p}}$ isotherm in figure 2. Deviations of about 1 percent were found for internal energy (fig. 3) and enthalpy (fig. 4). Deviations of a few tenths of a percent were found for entropy (fig. 5), density (fig. 6), and sound speed (fig. 7). The largest deviations in property values occurred for isotherms at 90, 100, and $110 \mathrm{~K}$ and at pressures near the two-phase boundary, for which the change in sound speed with pressure is largest.

The sound-speed measurements (ref. 3) from which the virial coefficients are computed were taken for isotherms from 80 to $350 \mathrm{~K}$ and at pressures from 0.33 to $15 \mathrm{~atm}$. Figures 8, 9, and 10 show sound-speed deviations at 80, 150, and $310 \mathrm{~K}$, respectively, computed with the virial coefficient as the reference. These three figures illustrate the accuracy of the three equations - virial, Jacobsen, and Beattie-Bridgeman - for predicting the experimental sound speeds, and that no one of these equations is clearly superior in terms of fitting the sound speed for isotherms over the entire temperature range. However, as explained later, the virial equation is expected to be superior in predicting specific heat capacities.

For figures 8 to 10 , the largest deviations of predicted sound speeds are at $150 \mathrm{~K}$. Sound speeds calculated with the virial equation are 0.1 to 0.2 percent larger than those calculated from either the Beattie-Bridgeman or the Jacobsen equation. For the $80-\mathrm{K}$ isotherm the deviations are in the other direction and become larger as the pressure increases, with the largest deviations occurring for the experimental data. The last three data points are believed to be incorrect, since they could not be represented by a virial expansion for this isotherm taken separately. However, comparison of virial surfaces computed with and without these points shows that the points do not affect the derived surface to any degree of significance. At temperatures larger than $150 \mathrm{~K}$ the agreement with the virial surface was generally as seen in figure 10 .

\section{ACCURACY OF THE CALCULATED VALUES OF THERMODYNAMIC PROPERTIES}

The accuracy of calculated values of thermodynamic properties depends on a number of different factors. Often an estimation of accuracy is made on the basic agreement between the experimental value and the calculated value of the property in question. An estimate made in this manner is an estimate of the precision of the experimental data and almost always results in an assessment of accuracy which is unrealistically small. Systematic errors in experimental data are usually greater than the imprecision in those data. 
A more realistic estimate of the accuracy of data is to compare those data with similar data from other sources and then make an estimate of the agreement. This method is probably conservative, but it is more realistic than equating precision to accuracy. In this particular case a realistic estimate of the accuracy of a property value may be determined by taking the maximum deviation shown for a particular property illustrated in figures 1 to 7 . The one exception is the specific heat capacity, for which experience has shown that empirical equations of state usually predict erroneous heat capacities near the saturated vapor boundary (and other boundaries such as the saturated liquid and solid boundaries) unless extreme care is taken to prevent the error (refs. 9 to 12 ). This erroneous heat-capacity behavior is due to the high degree of flexibility of most equations of state (the Jacobsen equation, for example, has 32 adjustable parameters) and the absence of the data immediately beyond the saturation boundary to control the behavior of the equation of state. In the case of the saturated vapor boundary, additional strain is placed on the equation of state because the equation must evaluate the region of densities between the vapor and liquid phases in such a manner as to predict the same pressure given the same temperature but different densities (i.e., a van der Waals loop). This problem is avoided by choosing the virial equation of state which has a minimum of adjustable constants and by using only vapor-phase data (no van der Waals loop required) with pressures of $15 \mathrm{~atm}$ or less.

Although multiproperty-fitting techniques used with $\mathrm{p}-\rho-\mathrm{T}$ data together with either sound-speed data or specific-heat-capacity data can reduce the error in specific-heat-capacity values as predicted by empirical equations of state near the two-phase transition, neither of these latter two kinds of data were available at the time the Jacobsen equation was formulated.

Based on the preceding arguments, the $c_{p}$ and $c_{v}$ from the wide-range equations of state near the saturated-vapor boundary are questionable. Therefore the maximum uncertainty of the virial $\mathrm{c}_{\mathrm{p}}$ and $\mathrm{c}_{\mathrm{V}}$ is estimated to be 2 percent, which corresponds to the disagreement shown in figures 2 and 3 for temperatures for which saturated conditions are not a factor. The average uncertainty in $c_{p}$ and $c_{v}$ is closer to 1 percent.

\section{SUMMARY OF RESULTS}

For many substances there is a lack of data in the gas region, especially near the two-phase boundary. As a consequence, the thermodynamic properties of this region are generally found to be poorly characterized by equations of state. To correct this situation for nitrogen, measurements of the speed of sound in the gas phase have been combined with $p-p-T$ data by multiproperty-fitting techniques to produce a density virial expansion for nitrogen. This new virial expansion should provide a more accurate representation of the thermodynamic properties (especially heat capacity) in this region.

The values of the virial coefficients found from this optimization are compared in detail to the thermodynamic-property values derived by Jacobsen. The virial equation sound speeds are also compared to the new experimental data and to those computed from the Beattie-Bridgeman equation and from the Jacobsen equation for $80-, 150-$, and $310-\mathrm{K}$ isotherms. 
Experimentalists should be encouraged to take more detailed data near two-phase boundaries, and especially in the gas region, since the behavior of the thermodynamic surfaces near these boundaries is generally the most uncertain. However, in the case of the gas phase this may well be the most difficult region to take data.

\section{Langley Research Center}

National Aeronautics and Space Administration

Hampton, VA 23665

November 2, 1979 
1. Jacobsen, R. T.; Stewart, R. B.; McCarty, R. D. ; and Hanley, H. J. M.: Thermophysical Properties of Nitrogen From the Fusion Line to $3500 \mathrm{R}$ $(1944 \mathrm{~K})$ for Pressures to $150000 \mathrm{psia}\left(10342 \times 10^{5} \mathrm{~N} / \mathrm{m}^{2}\right)$. NBS Tech. Note No. 648 , U.S. Dep. Com., Dec. 1973.

2. Beattie, James A.; and Bridgeman, Oscar C.: A New Equation of State for Fluids. II. Application to Helium, Neon, Argon, Hydrogen, Nitrogen, oxygen, Air and Methane. J. American Chem. Soc., vol. 50, no. 12, Dec. 1928, pp. $3133-3138$.

3. Younglove, Ben A.; and MCCarty, Robert D.: Velocity of Sound Measurements for Nitrogen Gas at Temperatures From 80 to $350 \mathrm{~K}$, and Pressures to $1.5 \mathrm{mPa}$. J. Chem. Thermo., vol. 12, 1980 (to be published).

4. McCarty, R. D.: Determination of Thermodynamic Properties From the Experimental p-V-T Relationships. Experimental Thermodynamics. Volume 2 Experimental Thermodynamics of Non-Reacting Fluids, Butterworth \& Co., Ltd., c.1975, pp. 501-526.

5. Gammon, B. E.: The Velocity of Sound with Derived state Properties in Helium at -175 to $150^{\circ} \mathrm{C}$ With Pressure to 150 atm. J. Chem. Phys., vol. 64, no. 6, Mar. 15, 1976, pp. 2556-2568.

6. Van Dael, W.; and Van Itterbeek, A.: The Velocity of Sound in Dense Fluids. Physics of High Pressures and the Condensed Phase, A. Van Itterbeek, ed., John Wiley \& Sons, Inc., 1965, pp. 297-357.

7. Quinn, T. J.; Chandler, T. R. D.; and Colclough, A. R.: Determination of the Gas Constant by an Acoustical Method. Nature, vol. 250, no. 5463, July 19, 1974, p. 218 .

8. Mylar - Physical, Electrical, and Chemical Properties. Tech. Rep. TR-1, E. I. du Pont de Nemours \& Co., Inc.

9. Roder, H. M.: The PVT Surface of Simple Liquids at Densities Near Melting. NASA CR-155757, 1977.

10. McCarty, R. D.: A Modified Benedict-Webb-Rubin Equation of State for Methane Using Recent Experimental Data. Cryogenics, vol. 14, no. 5, May 1974, pp. 276-280.

11. Roder, H. M.; and McCarty, R. D.: A Modified Benedict-Webh-Rubin Equation of State of Parahydrogen-2. Rep. COM-7511132/8, Nat. Bur. Stand., June 1975 .

12. MCCarty, R. D.: Thermodynamic Properties of Helium 4 From 2 to $1500 \mathrm{~K}$ at Pressures to $10^{8} \mathrm{~Pa}$. J. Phys. Chem. Ref. Data, vol. 2, no. 4, 1973, pp. 923-954. 


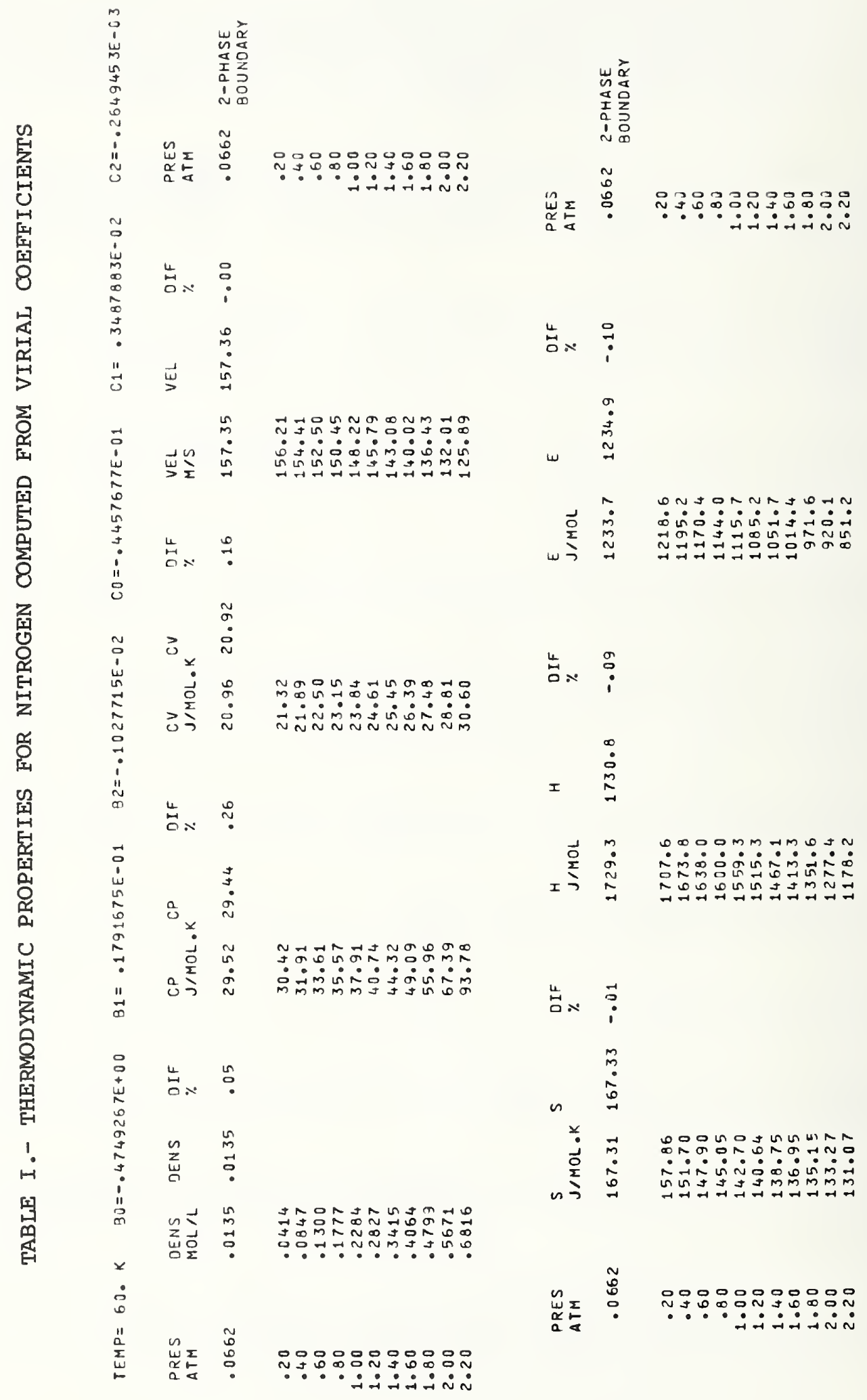




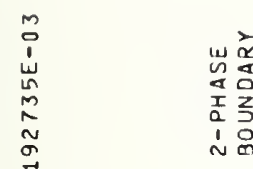

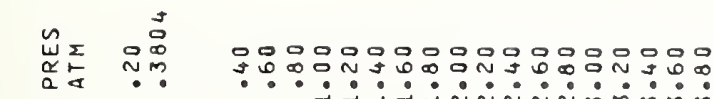

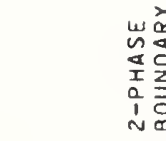

炭采

台.

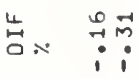

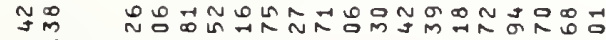

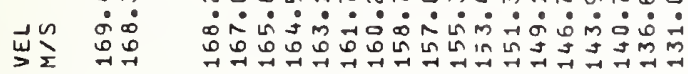

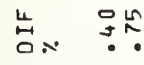

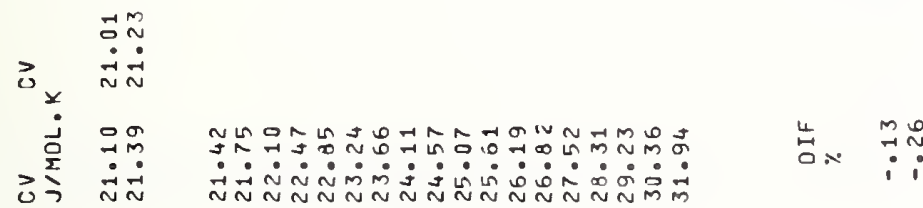

㟔メ

I

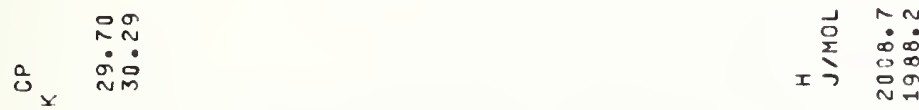

o.

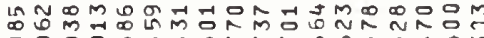

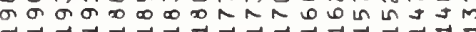

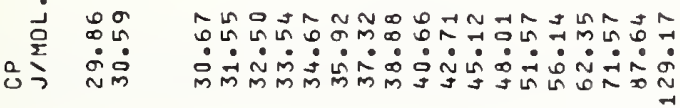

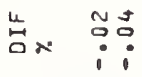

岃. ลัง

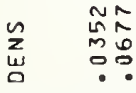

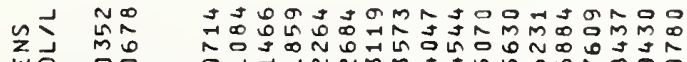

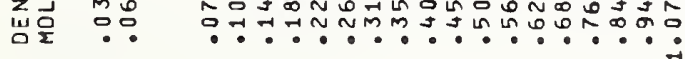

a

n.

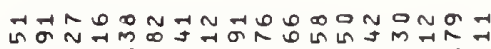

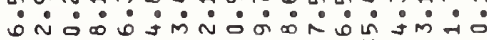

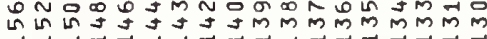

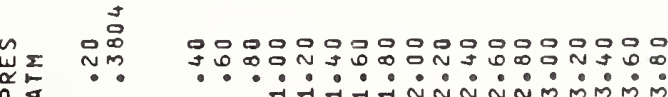

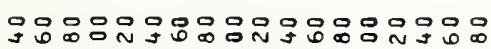

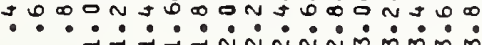




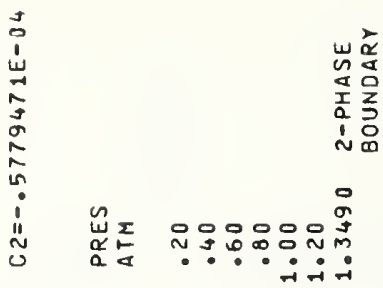

m

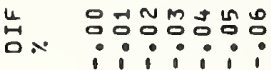

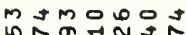

$\vec{\omega} \quad \dot{0} \dot{0} \dot{0} \dot{N} \dot{N}$

un

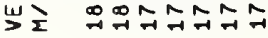

台. $\quad$ ก

'

+

约

1.

學
ํㅗㅇำ

\section{$\frac{\alpha}{\alpha}$

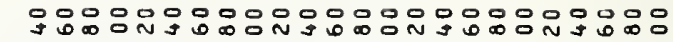
न

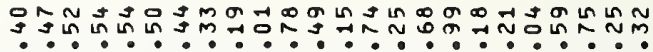

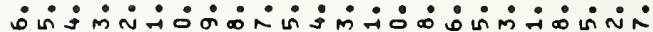

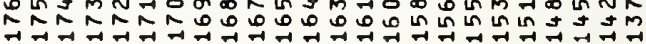

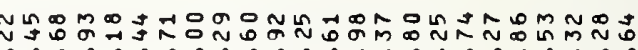

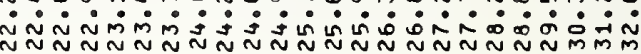

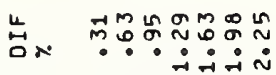

ษ

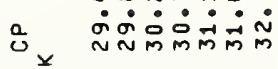

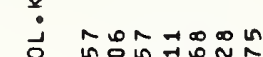

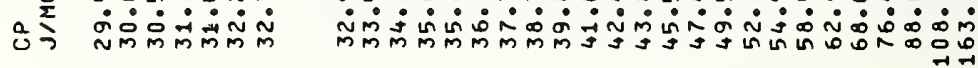

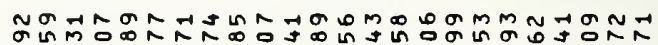
拧

苟.

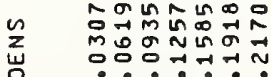

n

㟧实

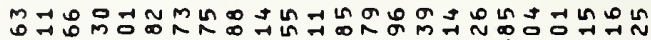

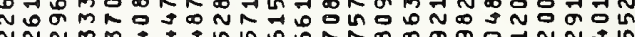

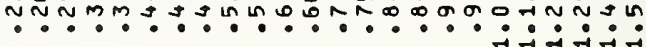

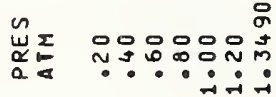

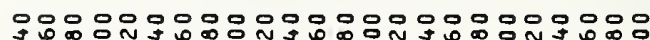

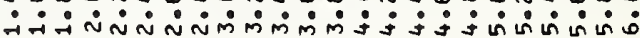




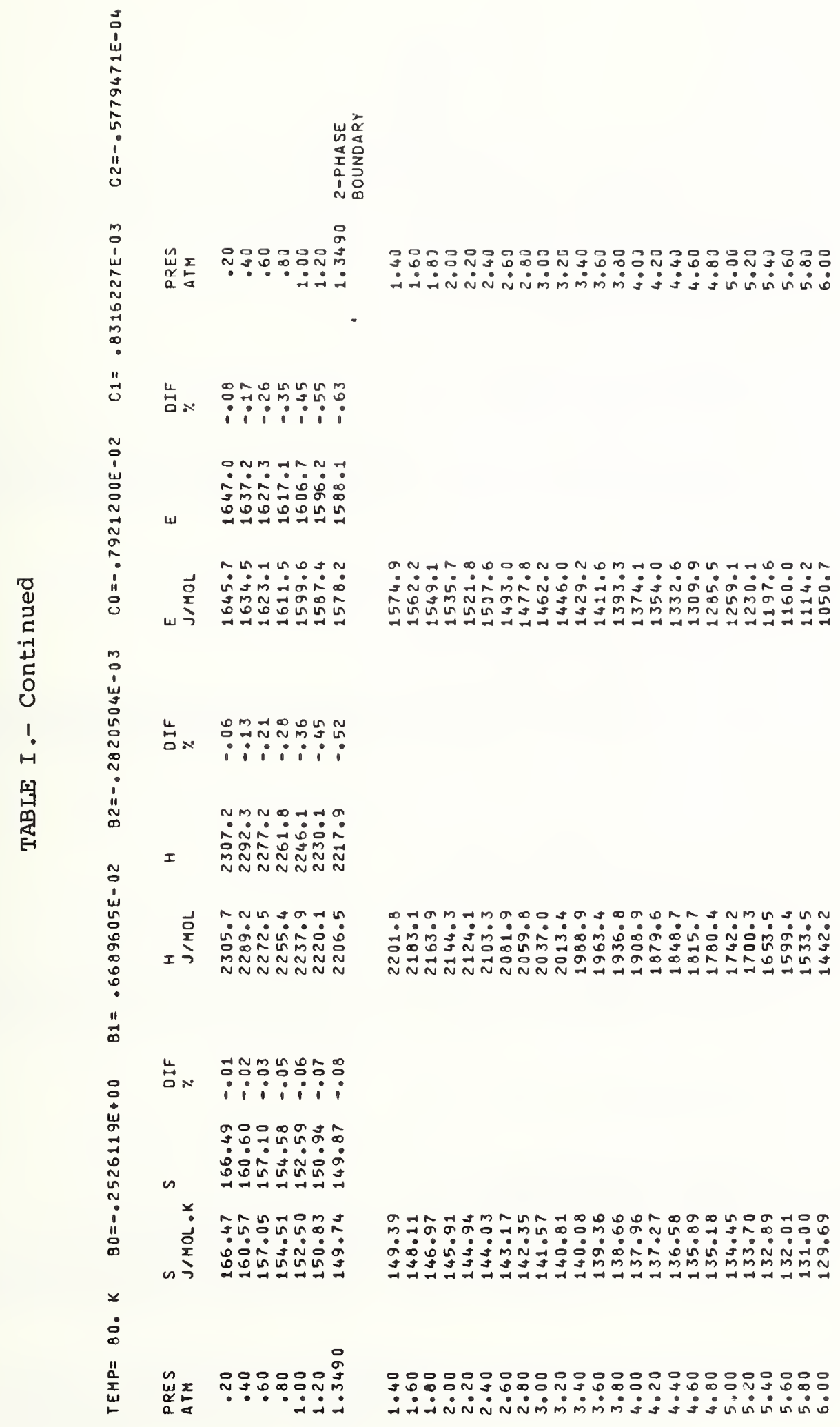




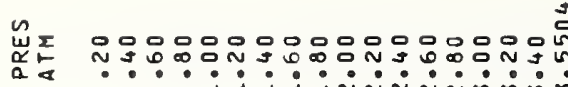

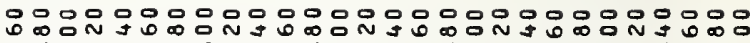

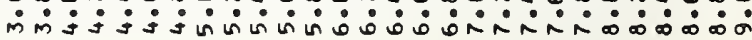

先

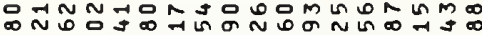

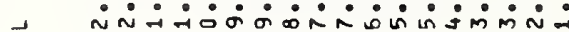

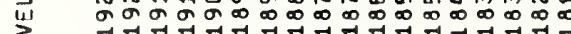

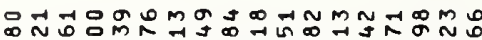

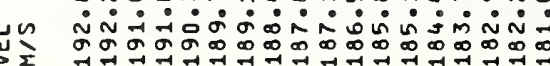

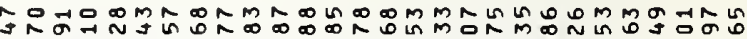

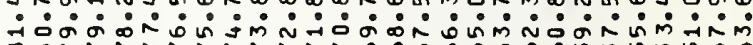

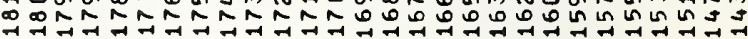

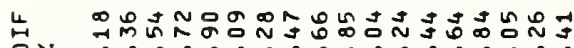

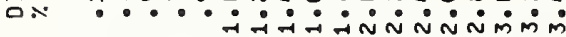

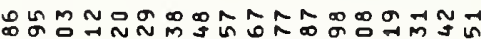

U $\quad \dot{N} \dot{N} \dot{N} \dot{N} \dot{N} \dot{N} \dot{N} \dot{N} \dot{N} \dot{N} \dot{N} \dot{N} \dot{N} \tilde{N} N \underset{N}{N} \dot{N}$

ดัล

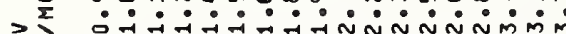

U:

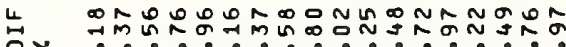

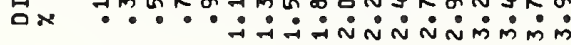

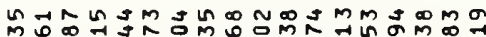

o

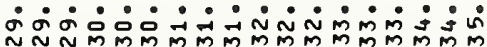

y.

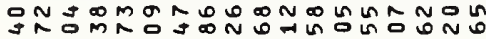

品

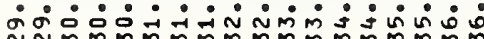

品

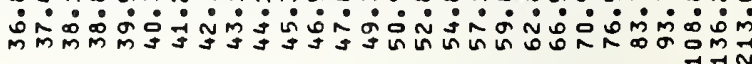

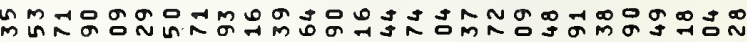

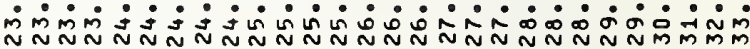

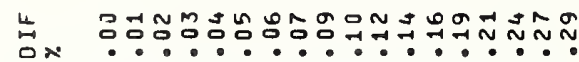

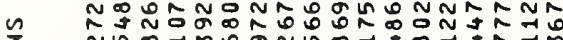

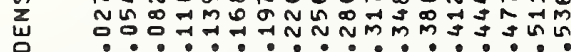

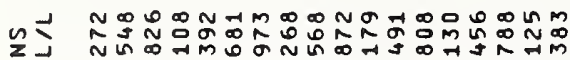

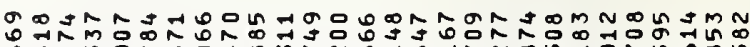

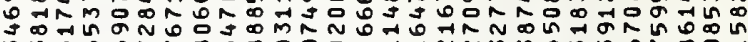
जी

놈오

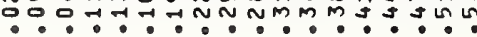

崖遂

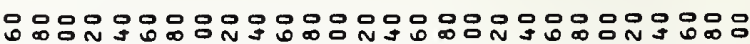

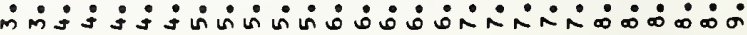




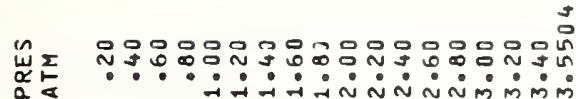

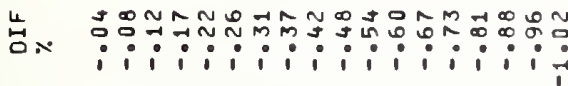

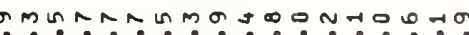

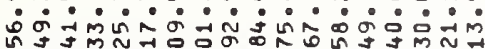

$\omega$

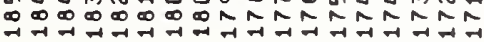

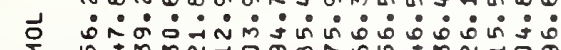
W

ㄴ. 品 : in

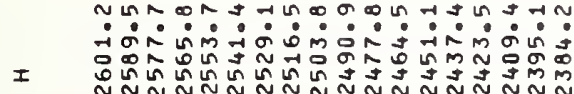

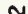

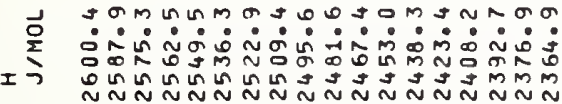

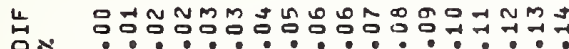

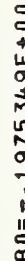

i i iliiii:iiiiiiii

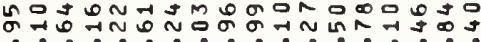
$\dot{\sigma} \dot{0} \dot{0} \dot{\sim} \dot{N} \dot{\sigma} \dot{\sigma} \dot{0} \dot{0} \dot{0} \dot{0}:$

n $\operatorname{lol}_{-1} 0$

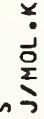

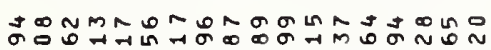

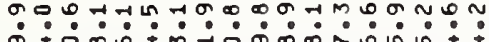

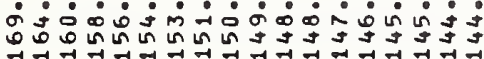

"I $\quad$ a

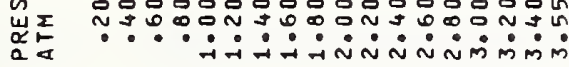

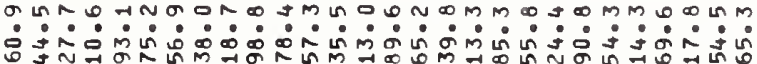

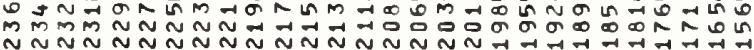

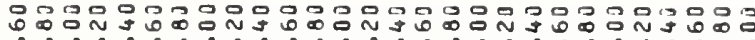

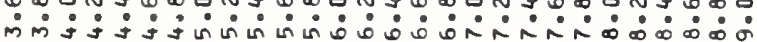

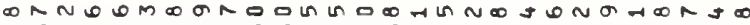

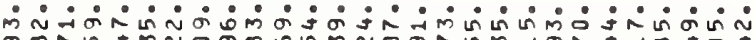

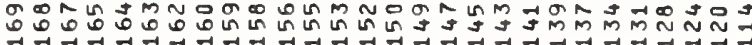

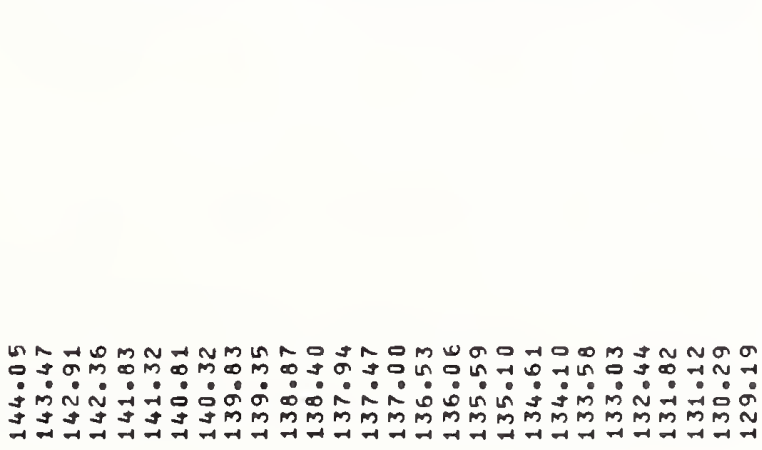

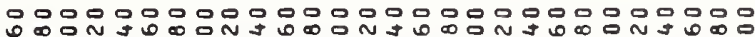

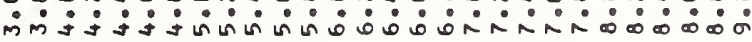




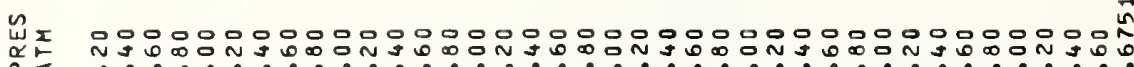

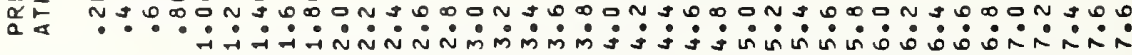

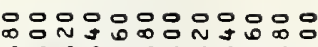

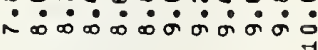

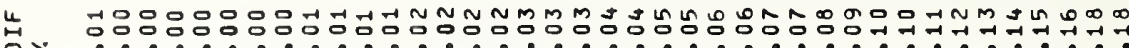

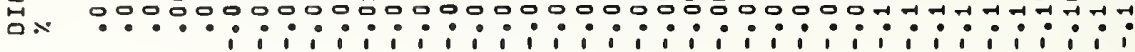

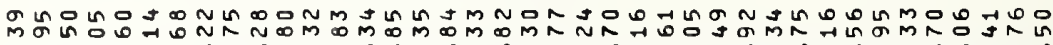
miñ

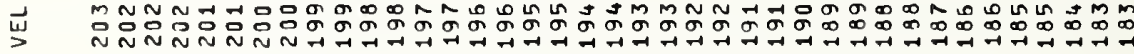

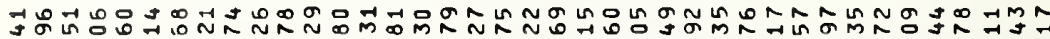

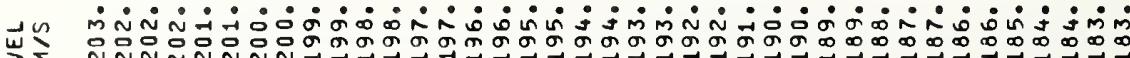

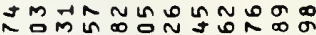

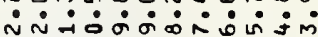

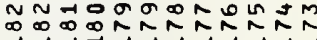

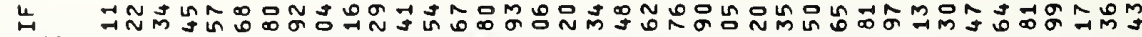

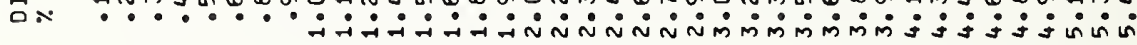

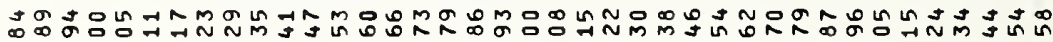
o

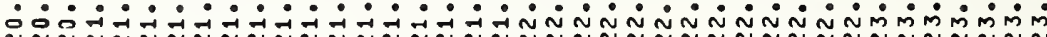
$\ddot{*}$

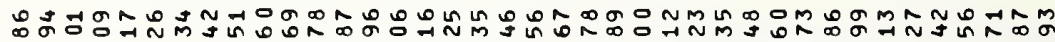

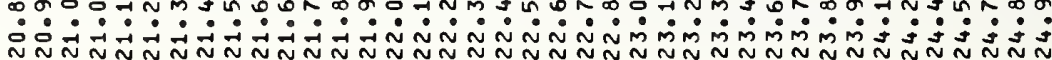

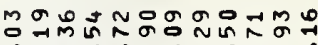

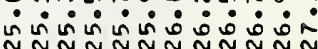

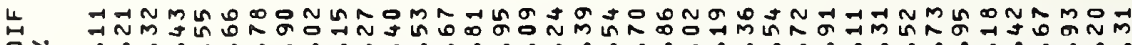

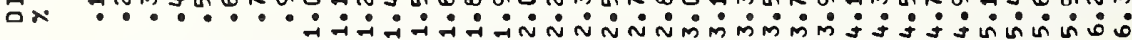

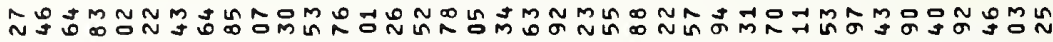

i

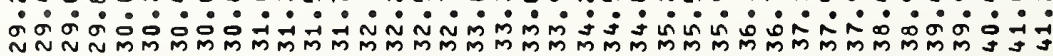

:

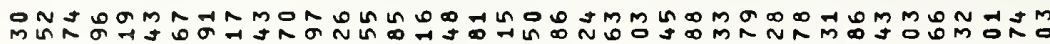
a)

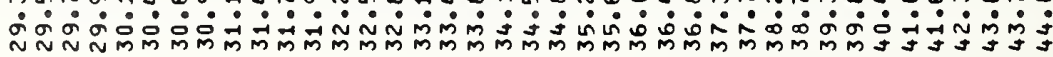

范 i i i i i i i i i

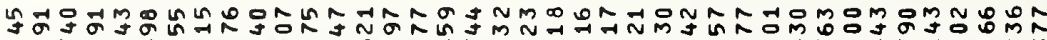

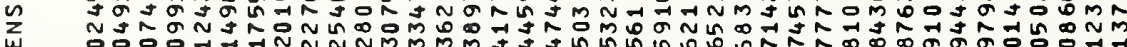

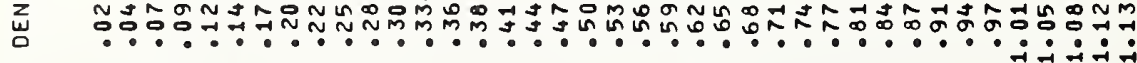

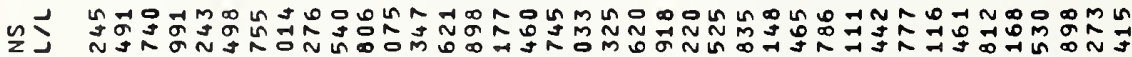

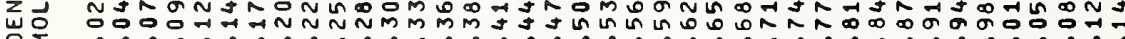

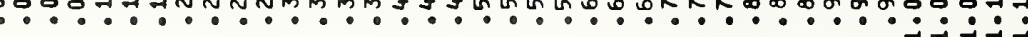

ที丶m

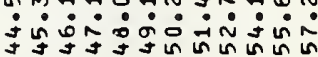

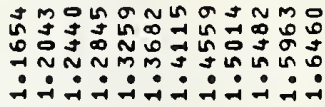

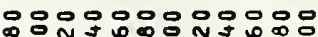

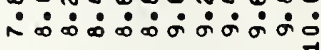




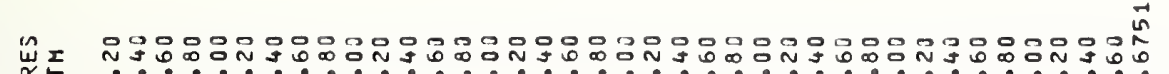

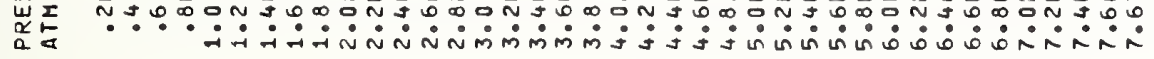

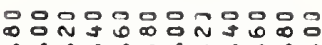

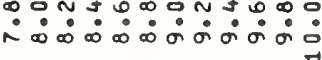

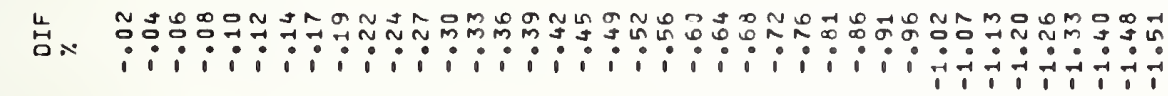

m

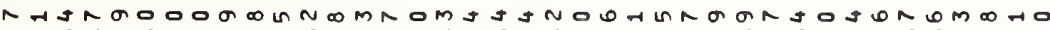

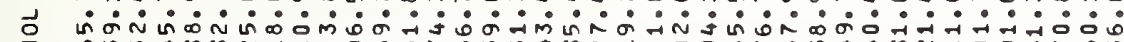
ए

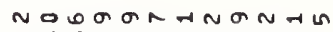

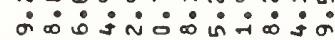
งกำ

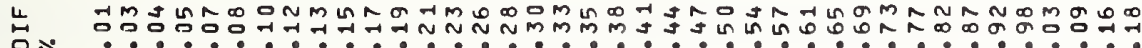

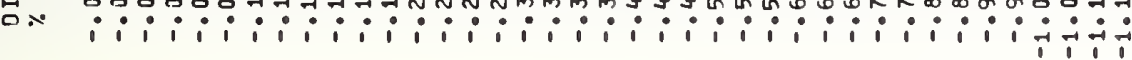

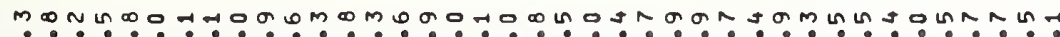

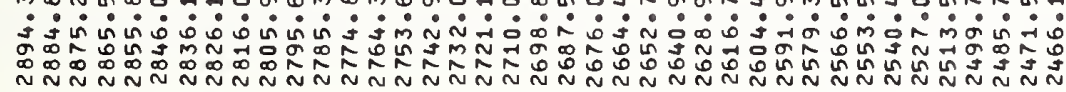

\section{I}

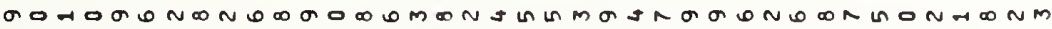

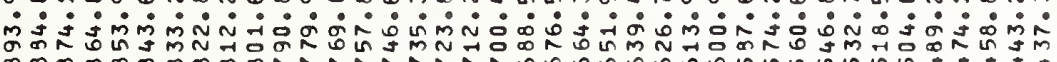

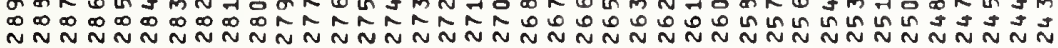

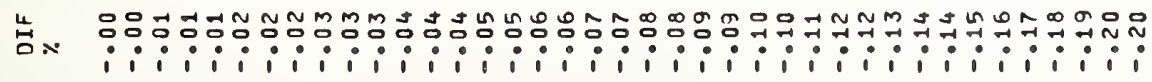

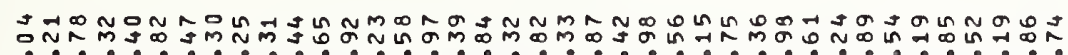

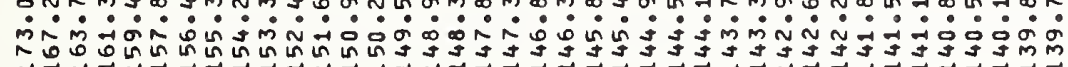

n

$\because$

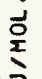

ind

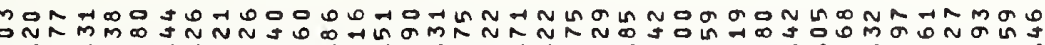

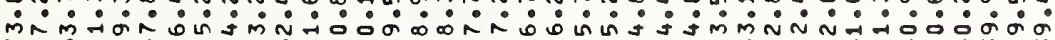

nว

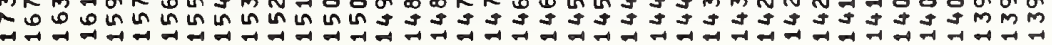

음

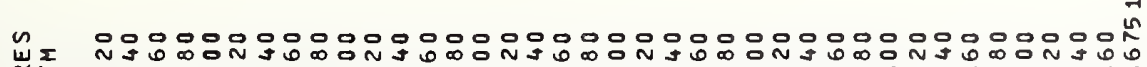
崖政

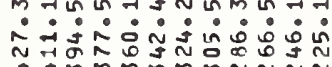
N N

\section{सहNล}

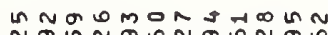
Non

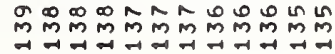

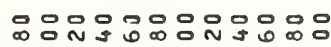

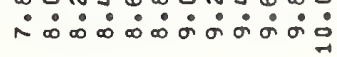


W I 00000000000000000000000000000000000000000000000000

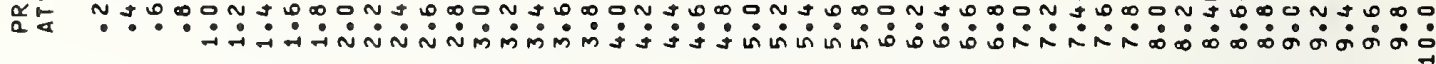

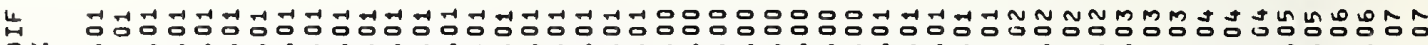

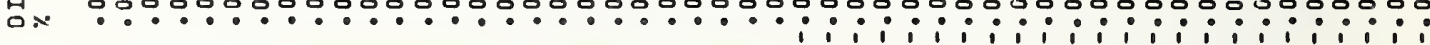
ป 0 -

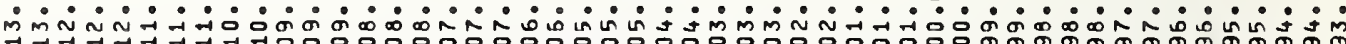

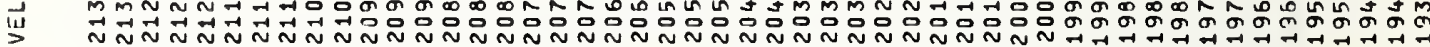

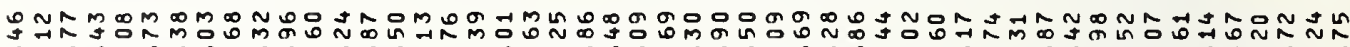

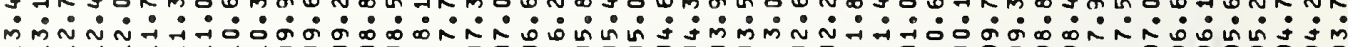

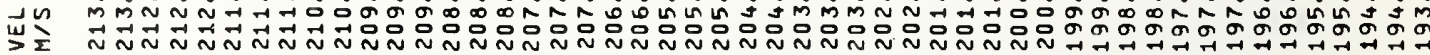

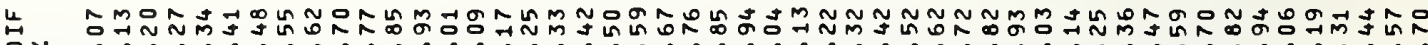

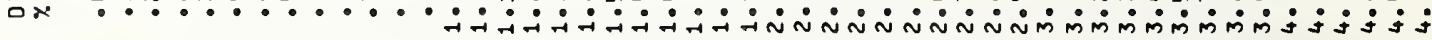
๗

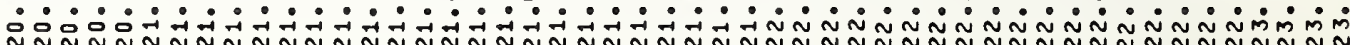

3 훌 3

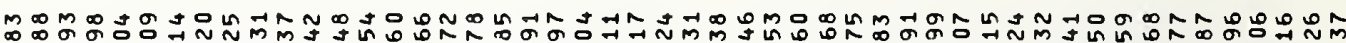

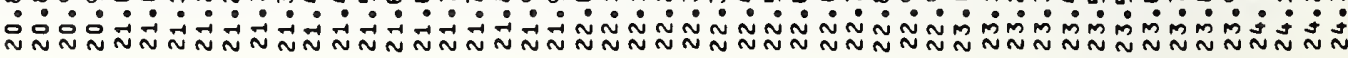

L 운

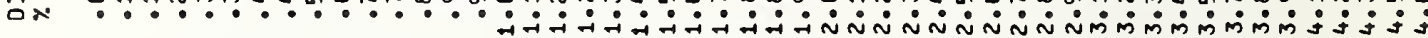

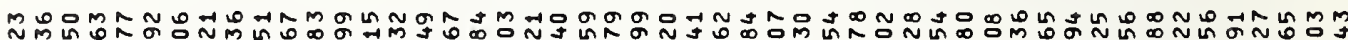

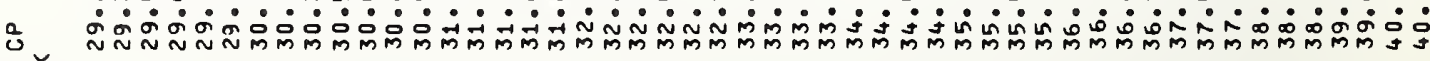

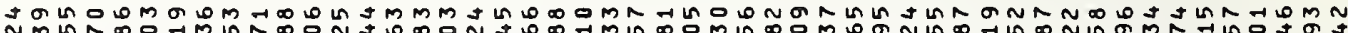

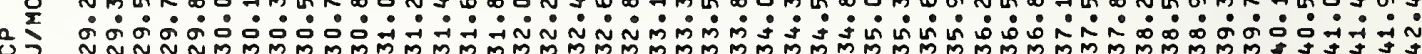

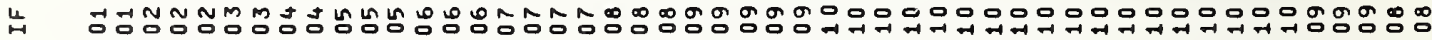

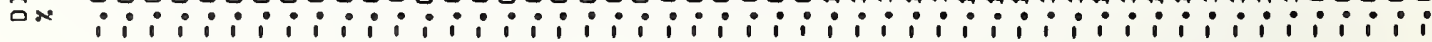

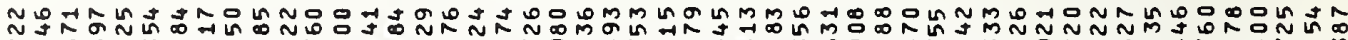

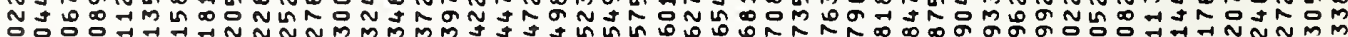
N N

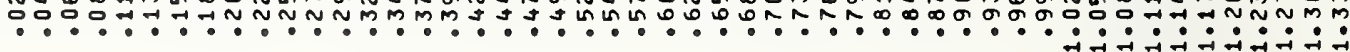

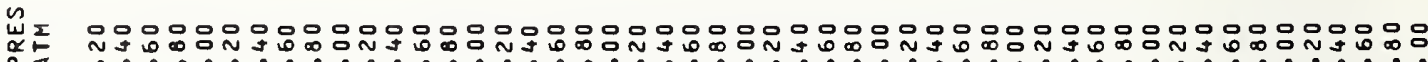

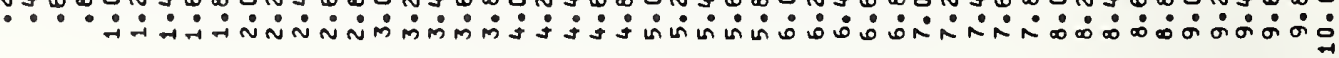


组

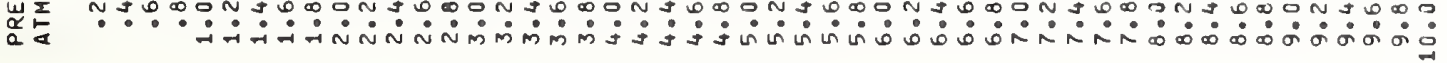

L 茨

Gนก N

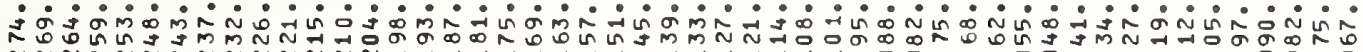

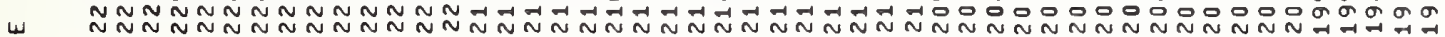

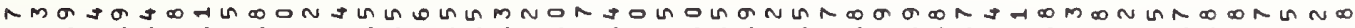

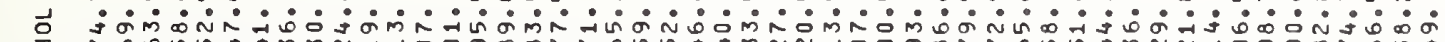

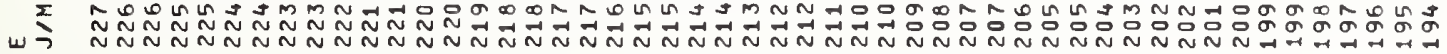

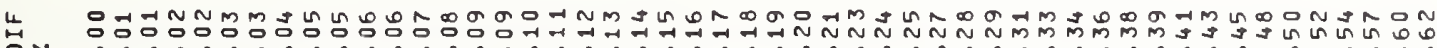
Le

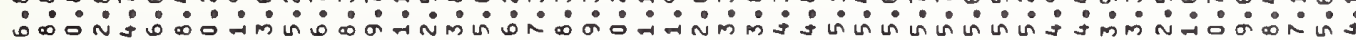

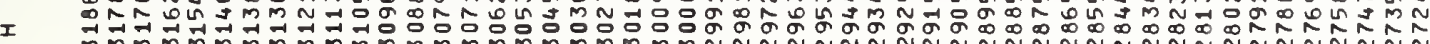

台

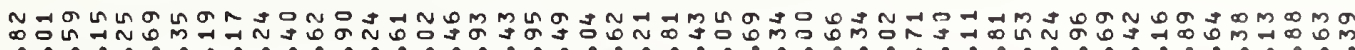

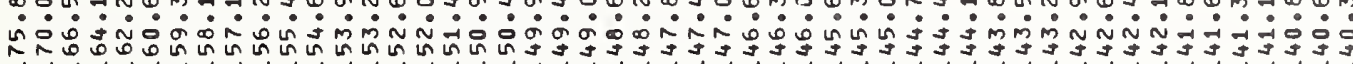

n

힐

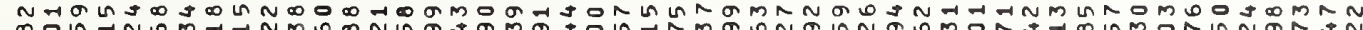

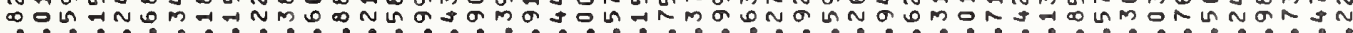

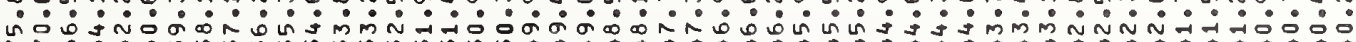

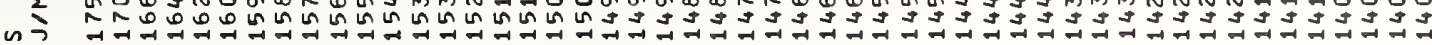

出 年正 
崫

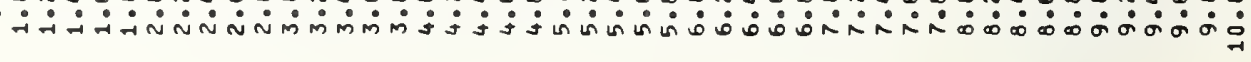

出 口

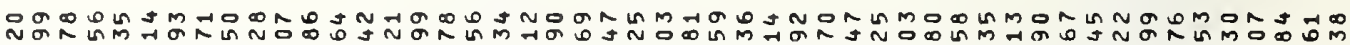

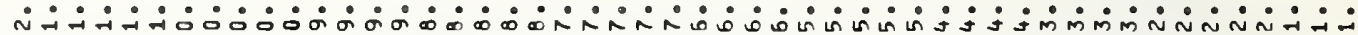

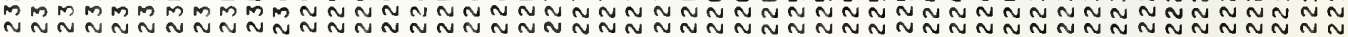

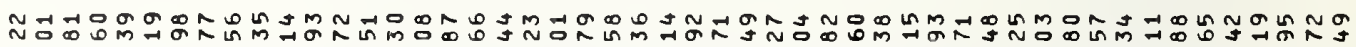

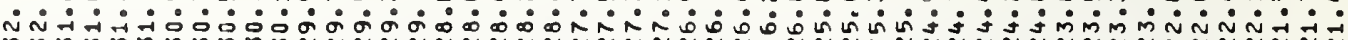

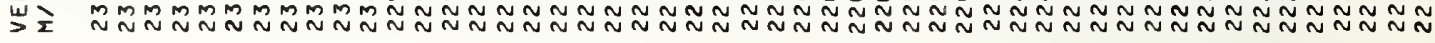

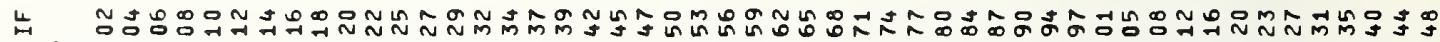
ON

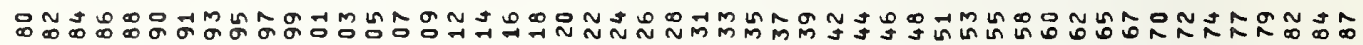

$\vec{u}$

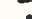

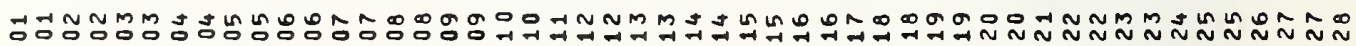

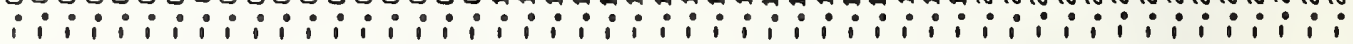

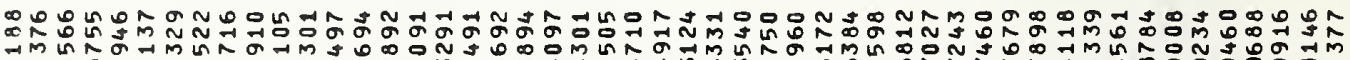

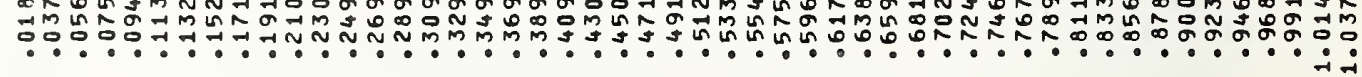

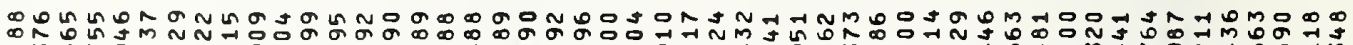

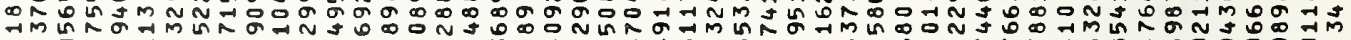

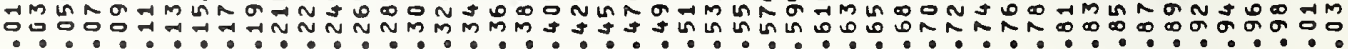

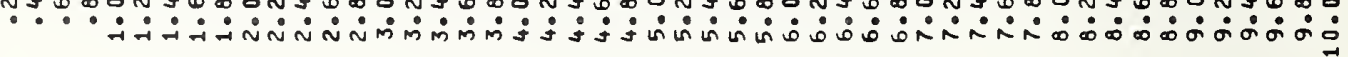


造

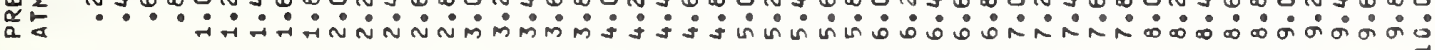

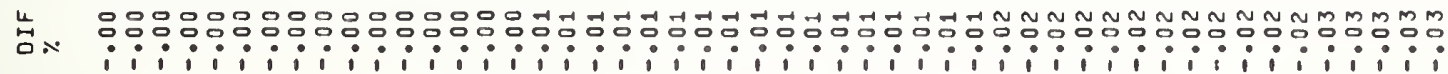

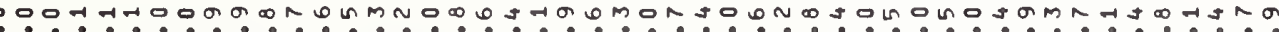

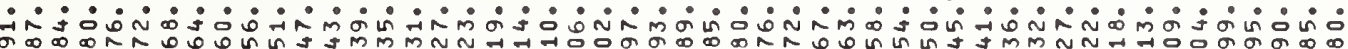

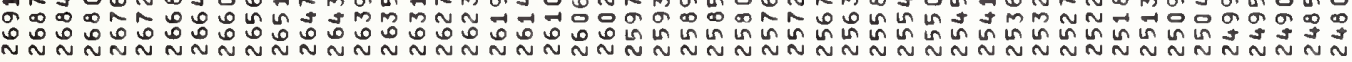

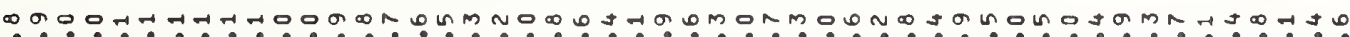

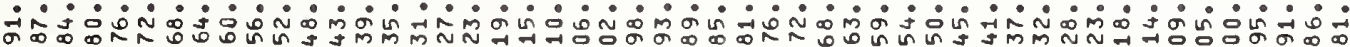

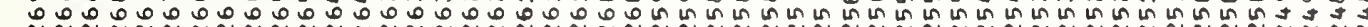

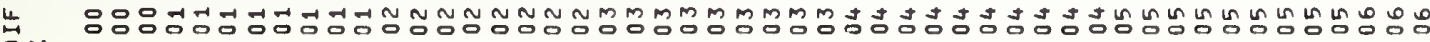
品

I $\hat{0}, 0.7-7 \pi 0$

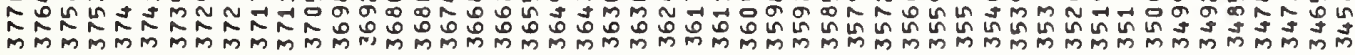

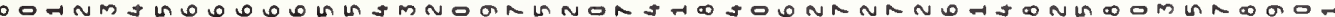

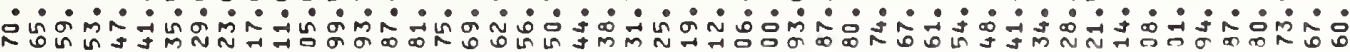

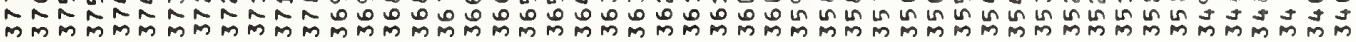

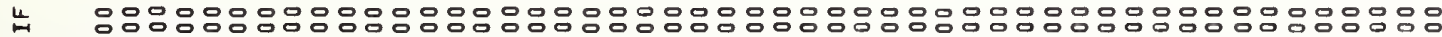

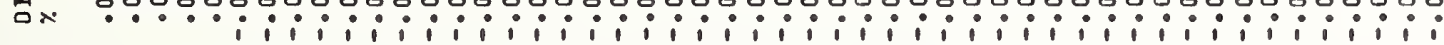
ำ大

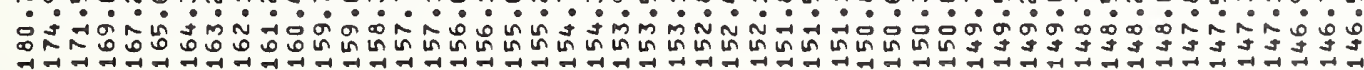

n

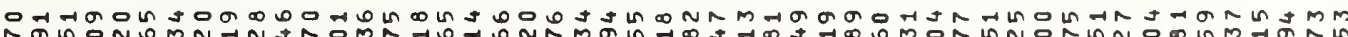

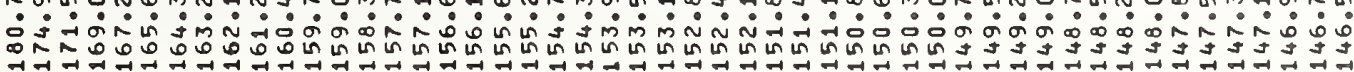




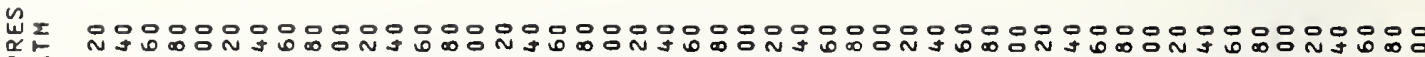
a

L

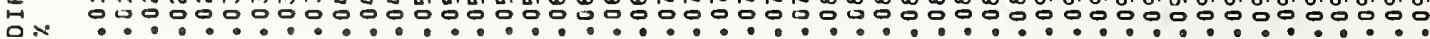

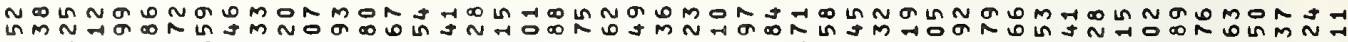

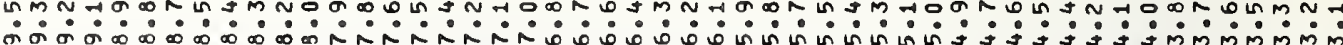

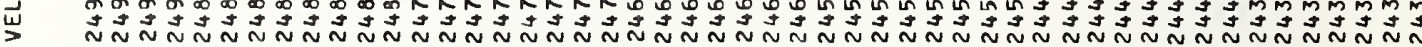

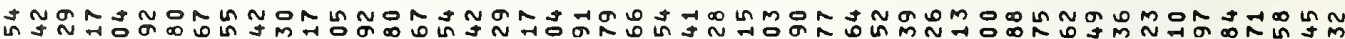
w

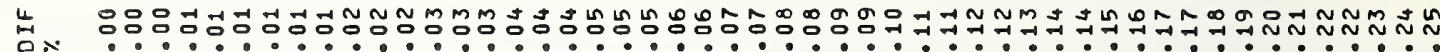

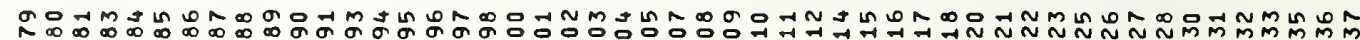
3

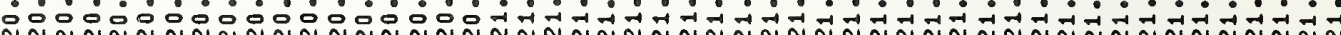

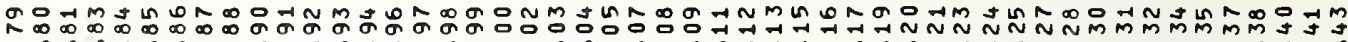
৩

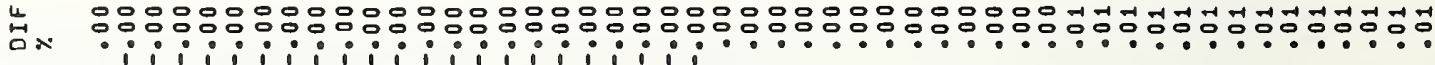

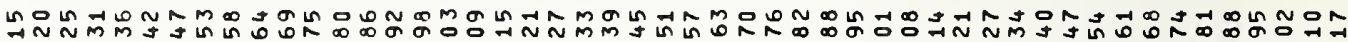
$\underset{.}{*}$

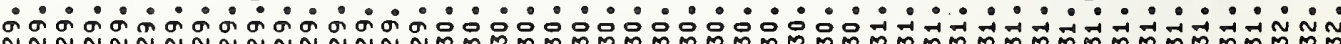
U

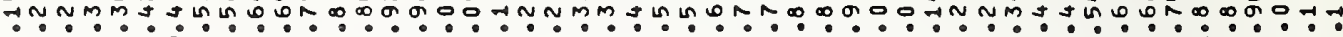

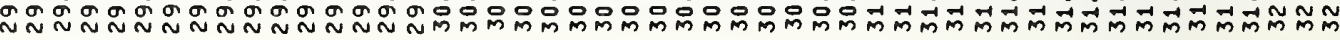

L ax

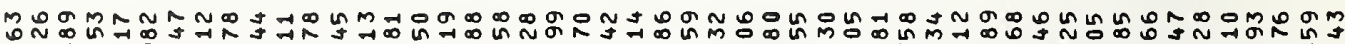
级

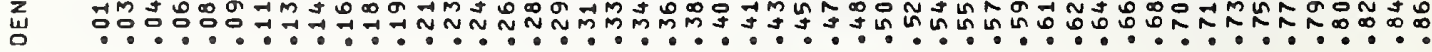

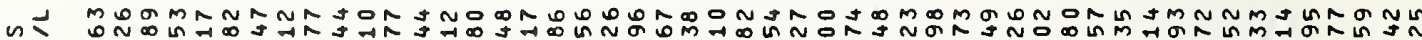
之 㟧定

嵌

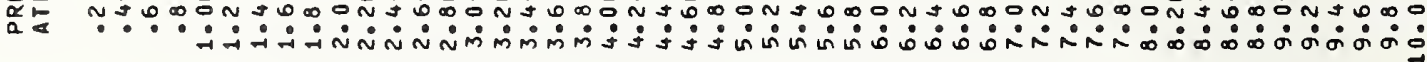




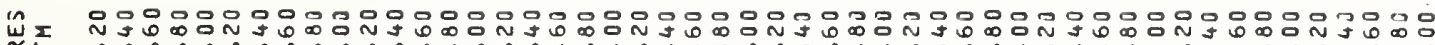
造

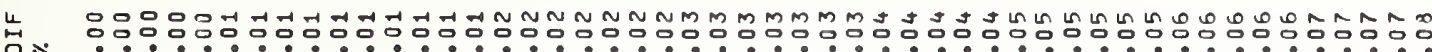

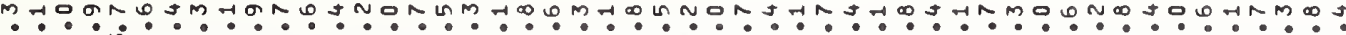
ळம்

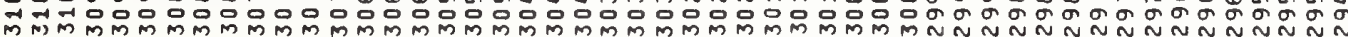

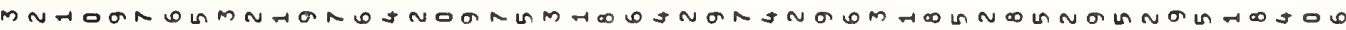

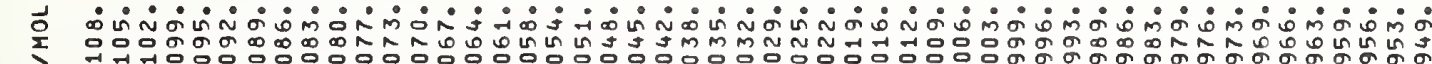
w

夜 OMMNG

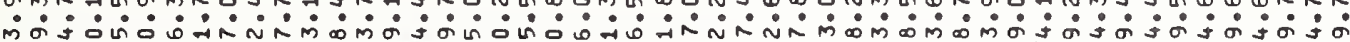

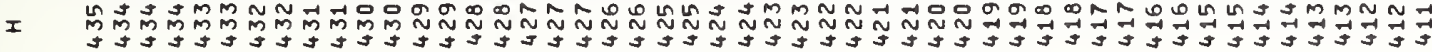

0 L

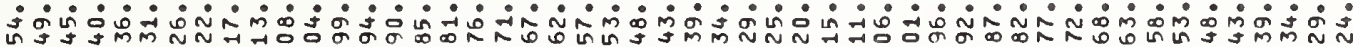

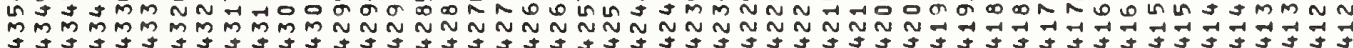

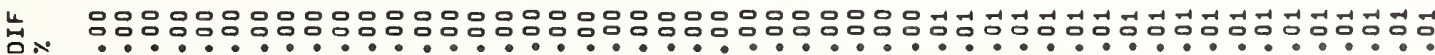

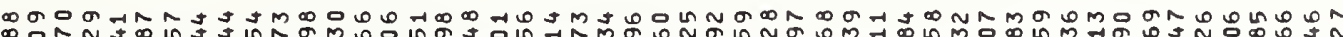
mor. ¡

n

$\times$ n

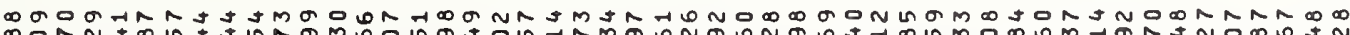

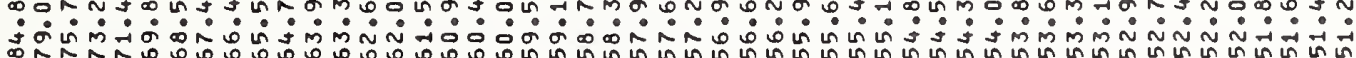
जन 出

20000000000000000000000000000000000000000000000000 


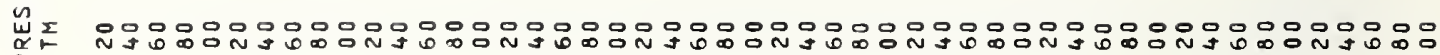
a

台

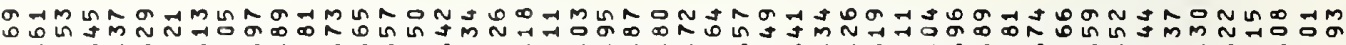

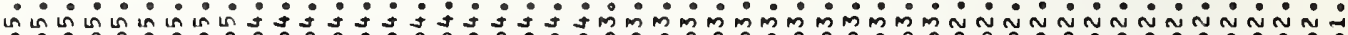
N N

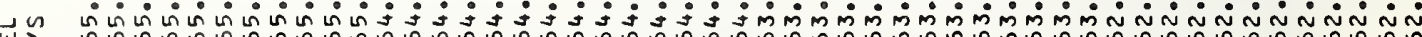

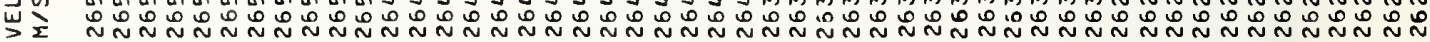

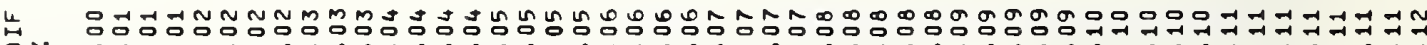

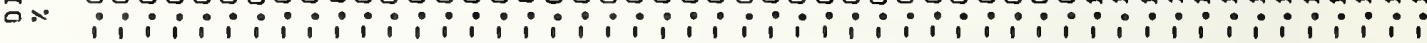

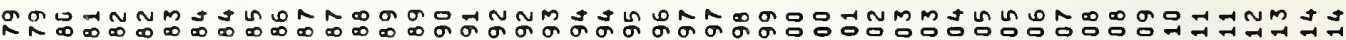
>

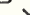

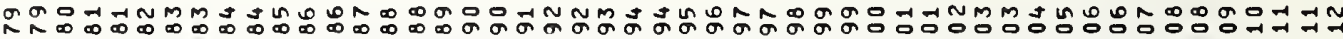

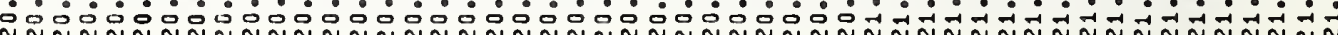

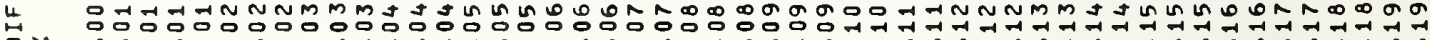

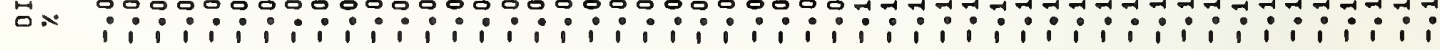

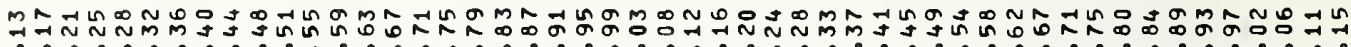

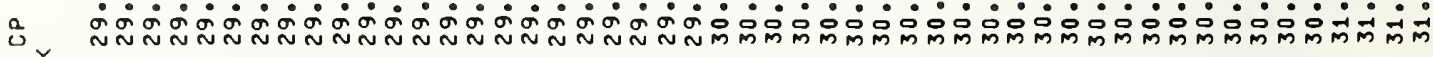

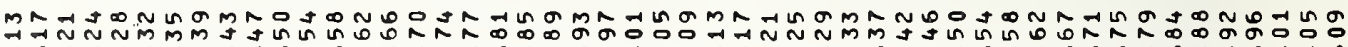
位

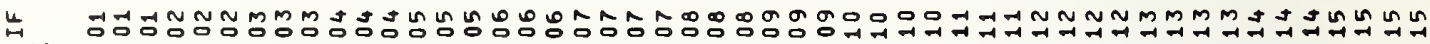
a

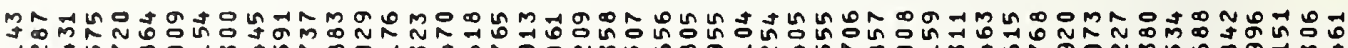

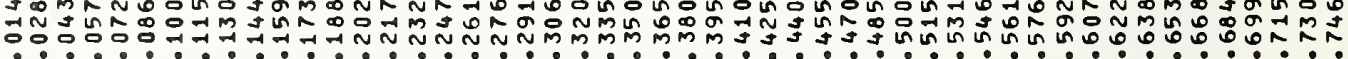
MN

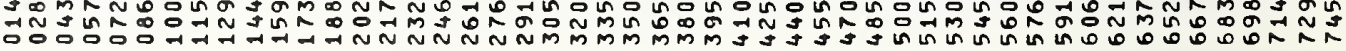

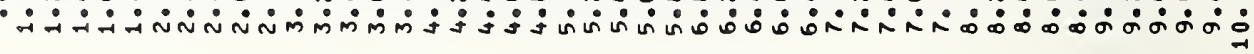




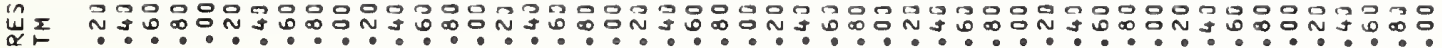

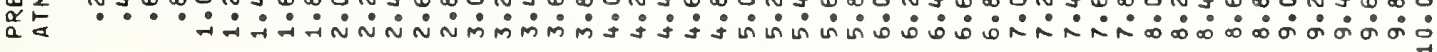

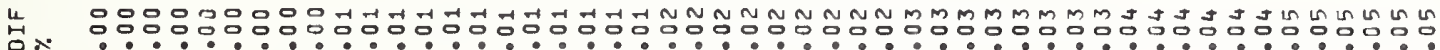
Ton

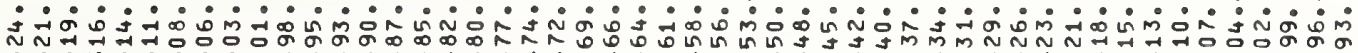

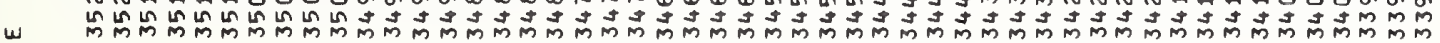

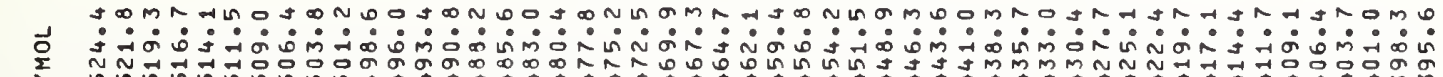

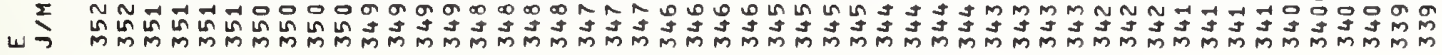

4.

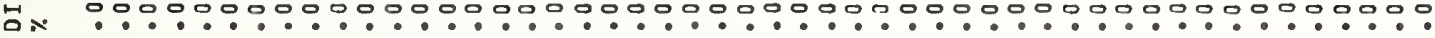

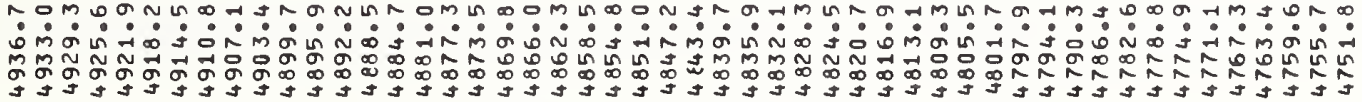

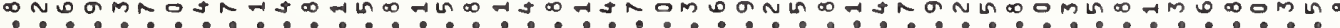

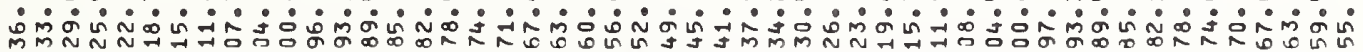

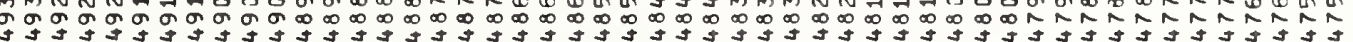

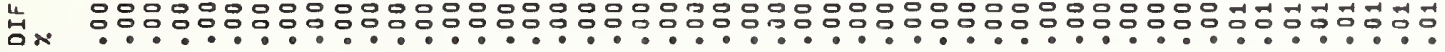
고ํำ

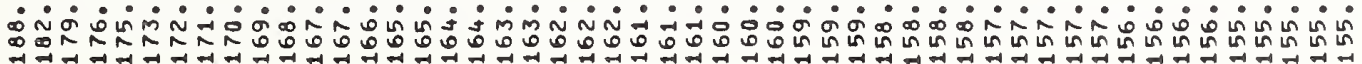

n × N

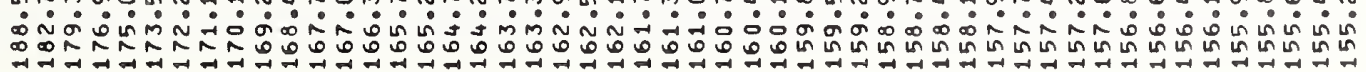




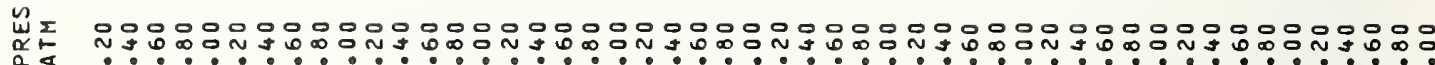

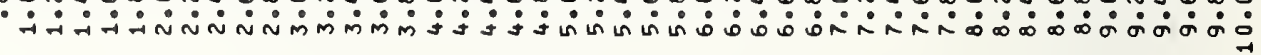

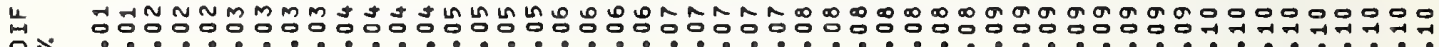

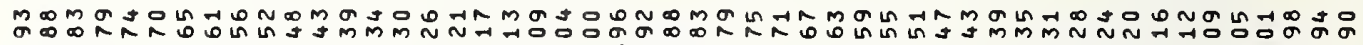

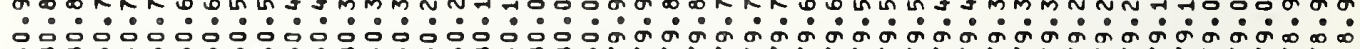

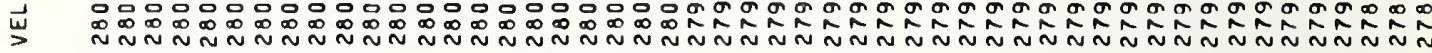

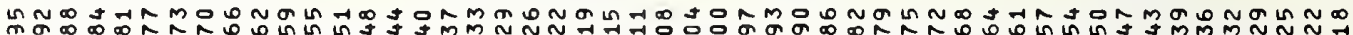

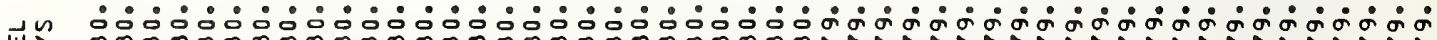

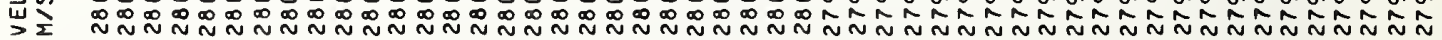

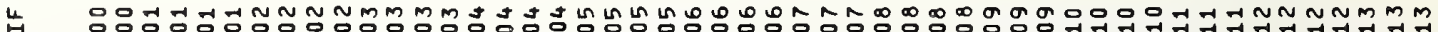

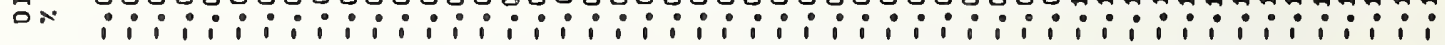

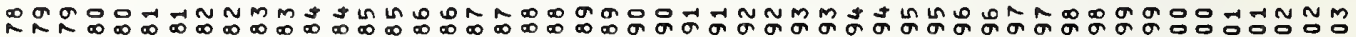
3

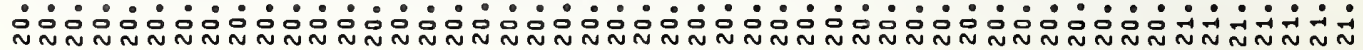

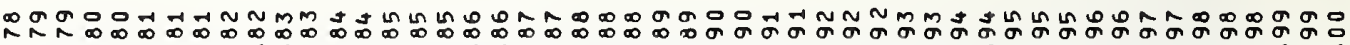

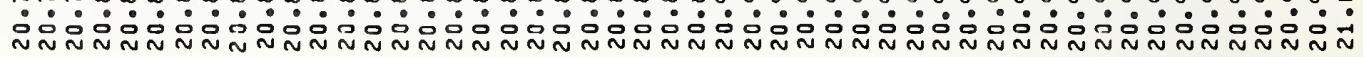

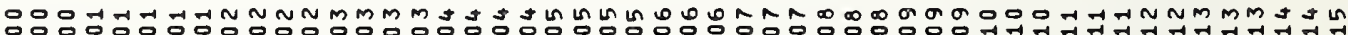
i:

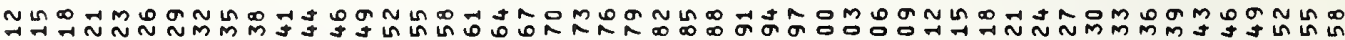
$\because 7$ ก N Nmm?

$\stackrel{a}{0}$

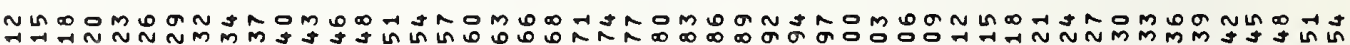

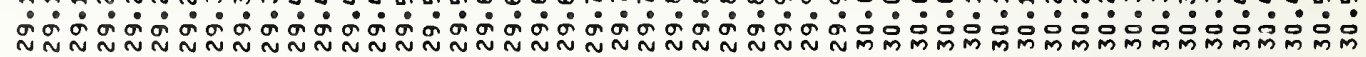

崫

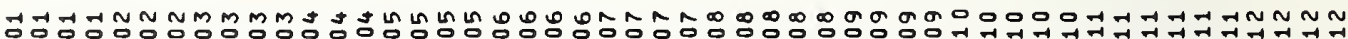
QN

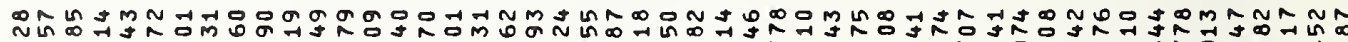
$\sum_{\text {岁 }}^{n}$

क人⿻上丨N

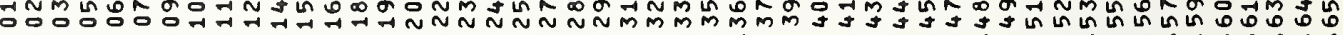

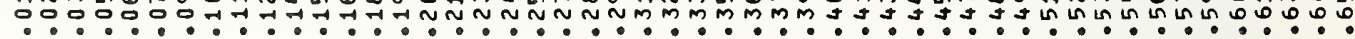

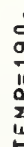

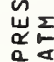

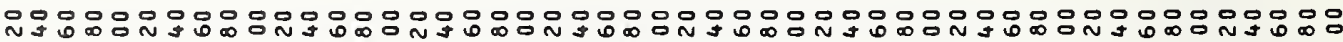

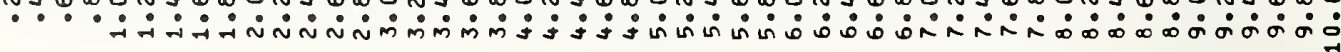


侸

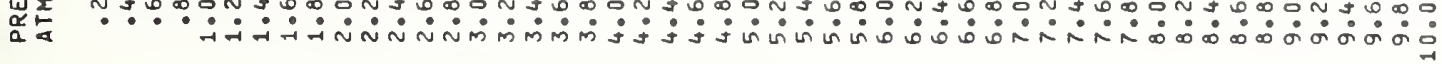

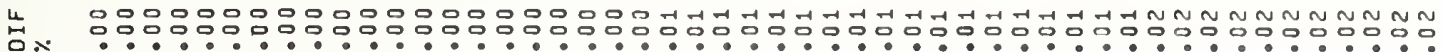

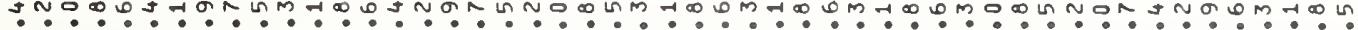

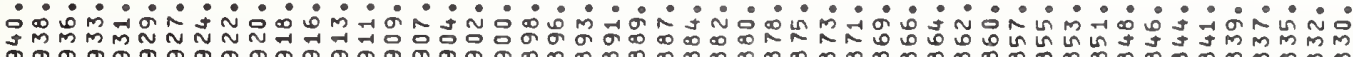

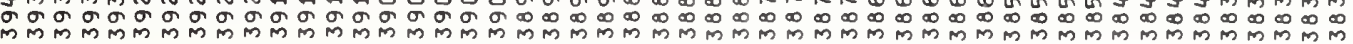

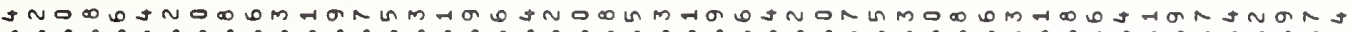

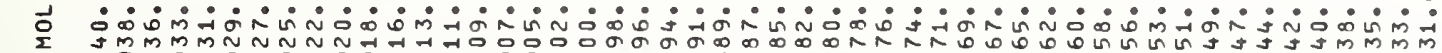

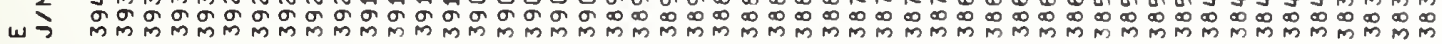

崖火 i⿱

I

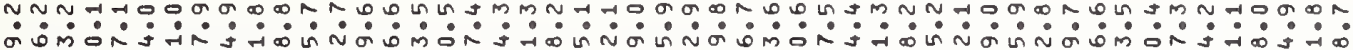

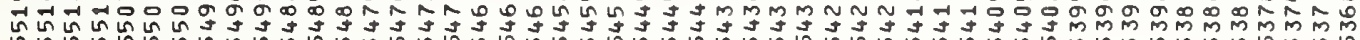

\section{ז}

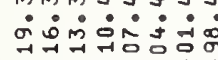

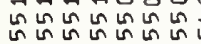

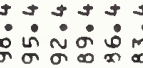

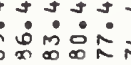

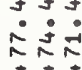

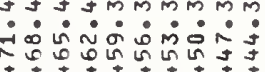

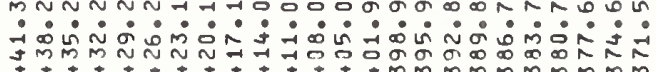

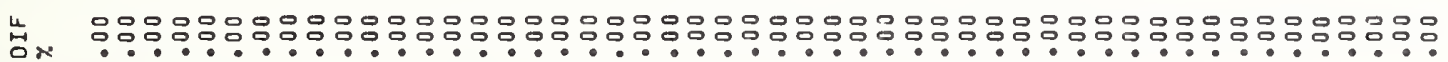

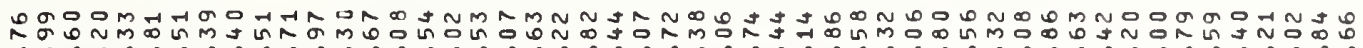

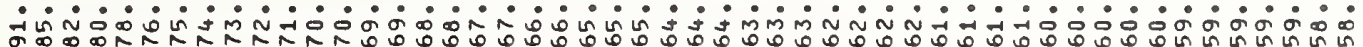

u

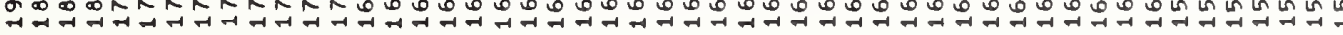

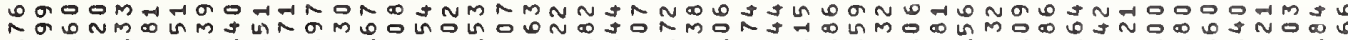

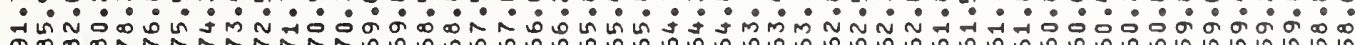

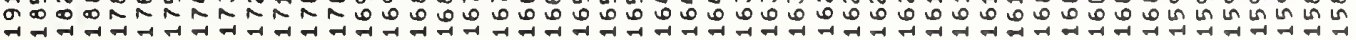

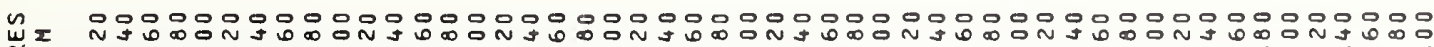

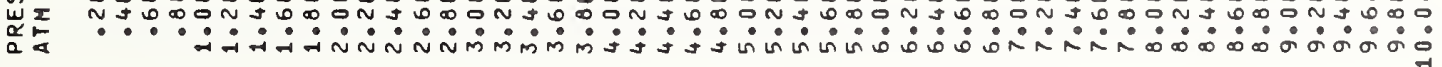


崫 -.

嵌

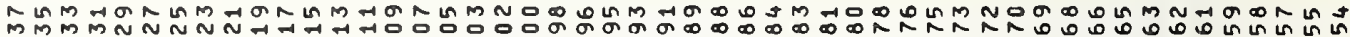

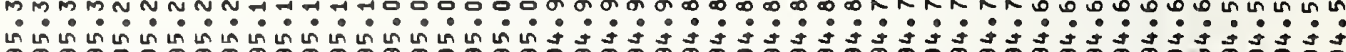
$\vec{w}$

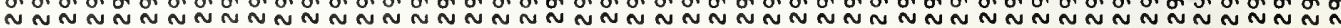

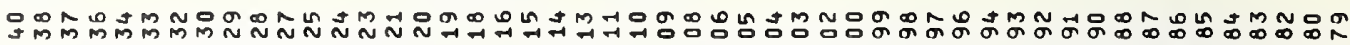

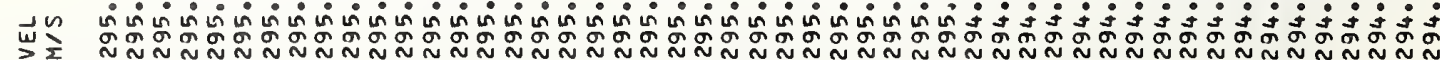

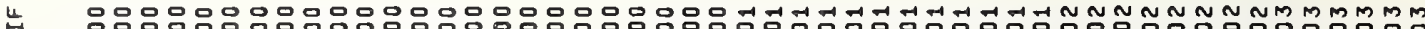
ON ................ i i i i i i i i i i i i i i i i i i i i i i i

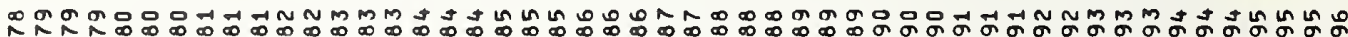

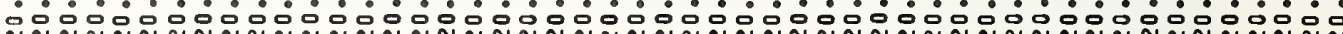

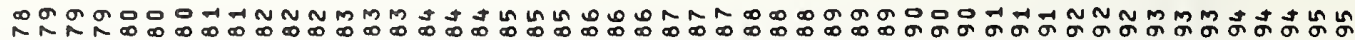

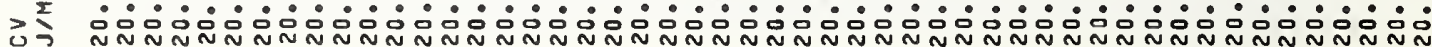

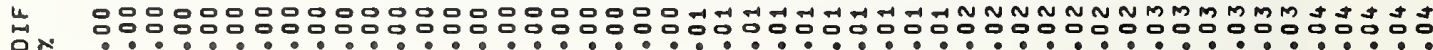
a

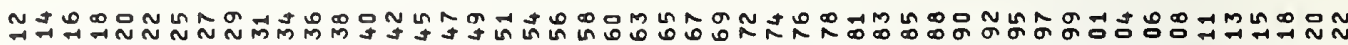
仓

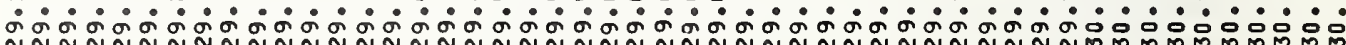
:

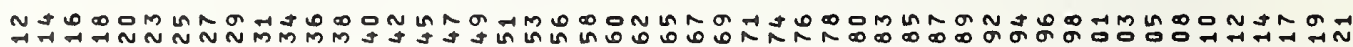
u $\quad$ i

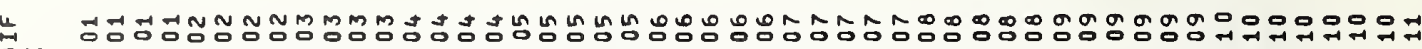
Ni i i i i i i i i i i i i i i i i i i i i i i i i i i i i i i i i i i i i

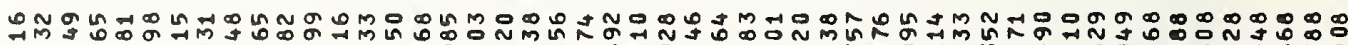

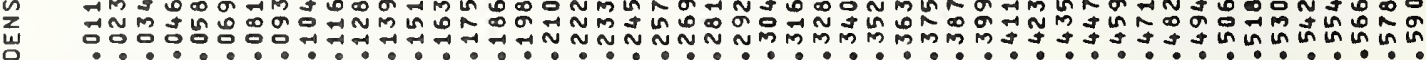
1

岁定

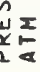

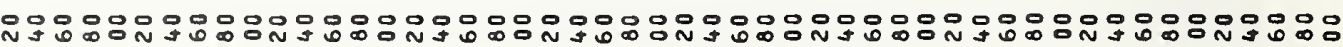

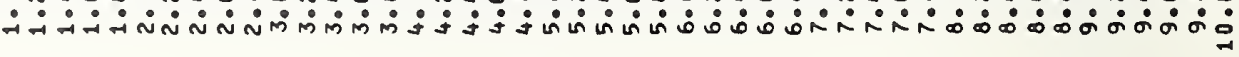


出 贻

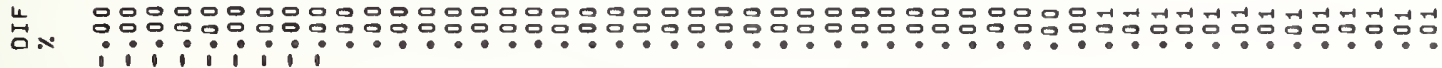

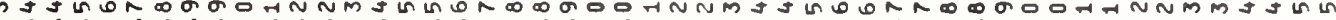

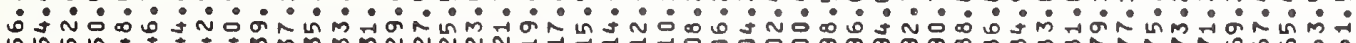
W

MMJUDN

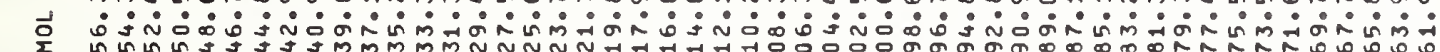
w

先

๑า

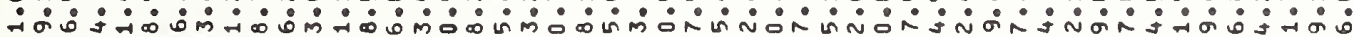

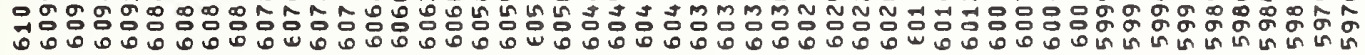

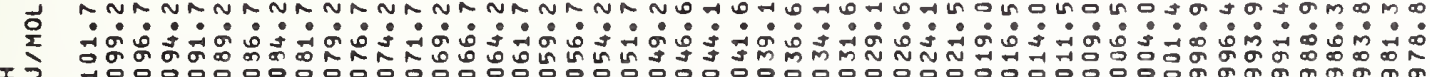
I

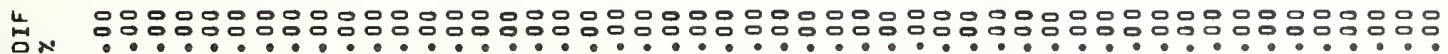

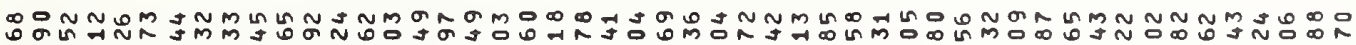

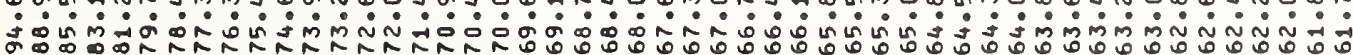

n

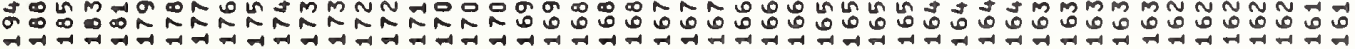

放

DON N

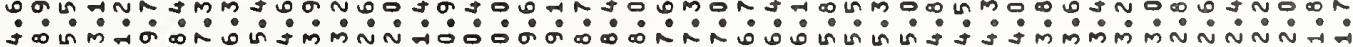

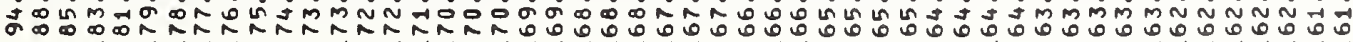

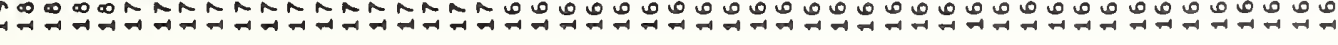

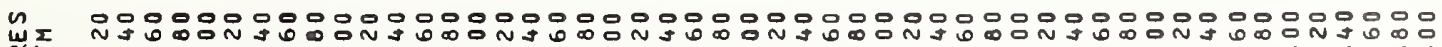
宏走 
凯 a a

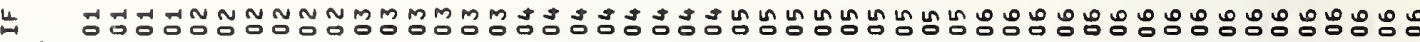

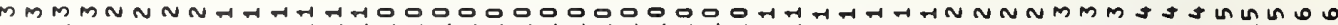
க்

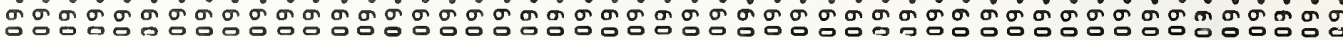

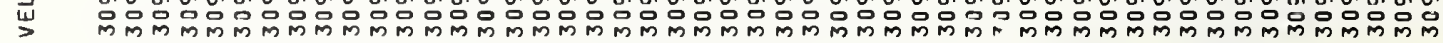

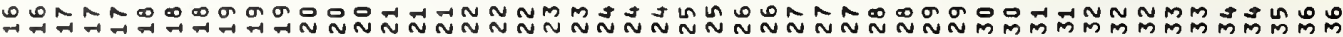

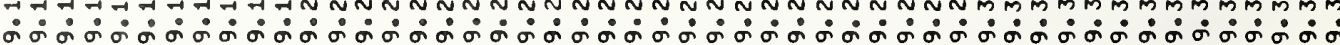

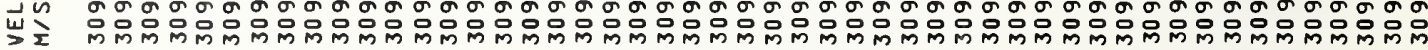

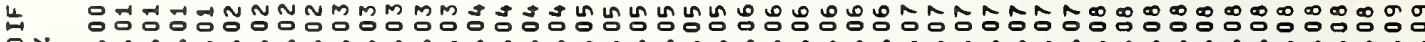

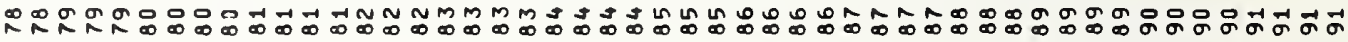
$\vec{u}$

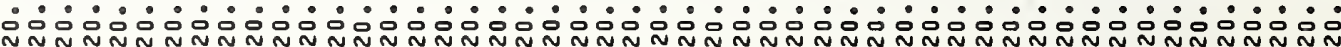

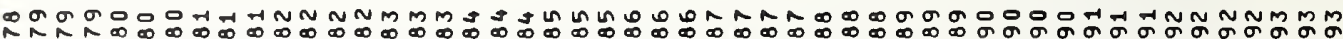

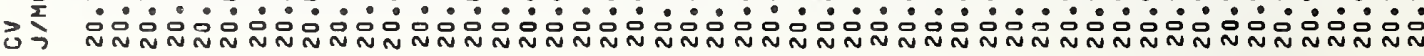

舀

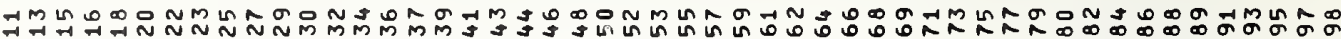

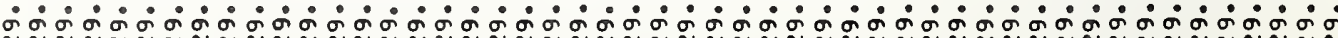
$x$

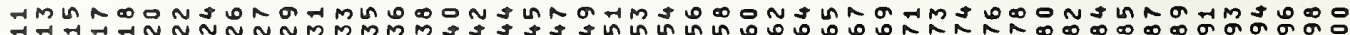

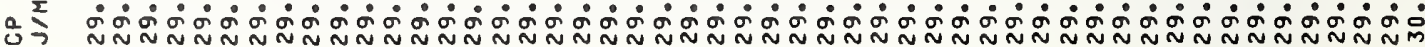

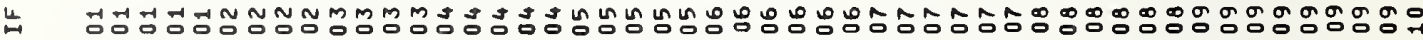

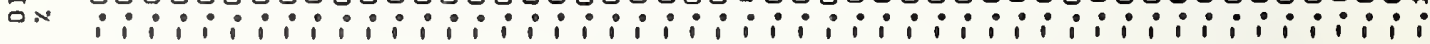
« 岁

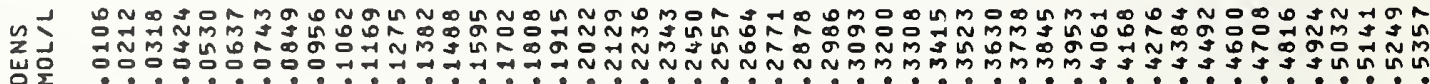




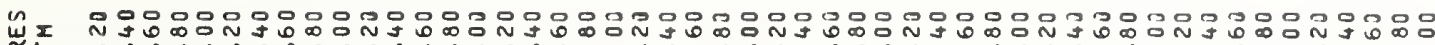
造

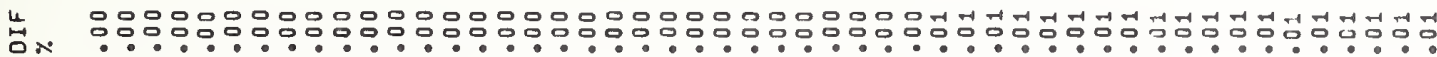

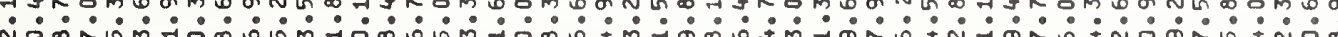
N m z a d a z

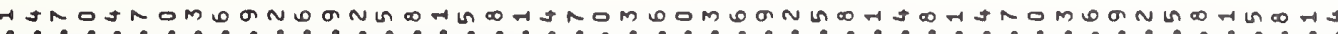

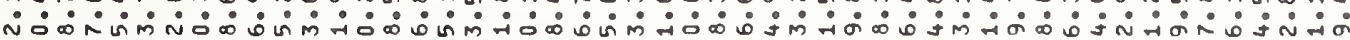
$j$
5
5
5
5

嵌 品

I máñ

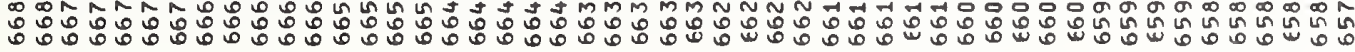

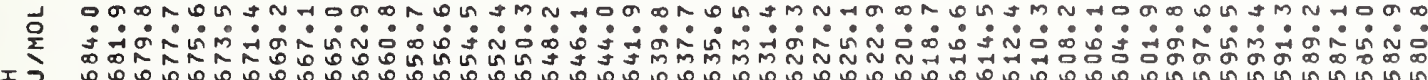

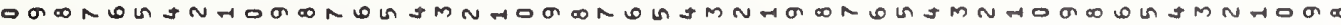

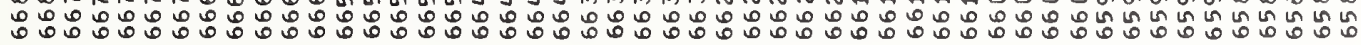

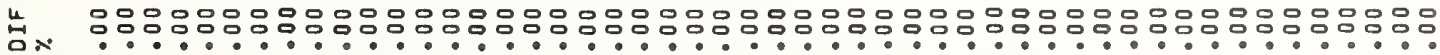
ผ

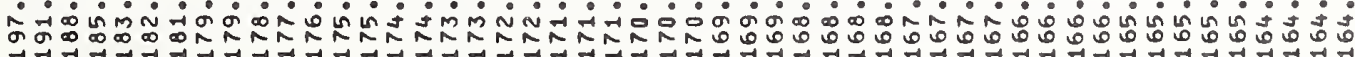

n

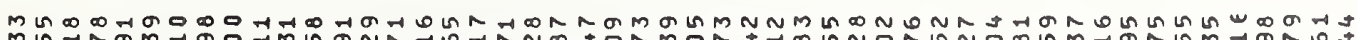

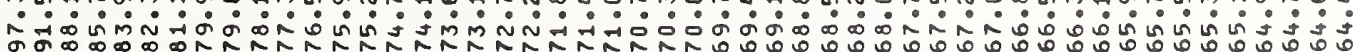
u

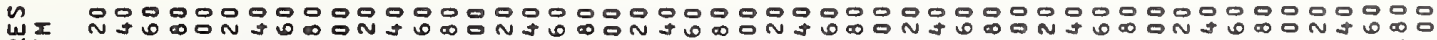
a 


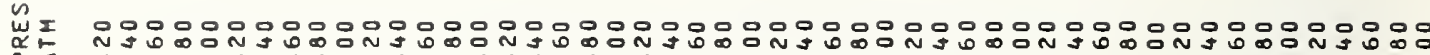
$\because \because 0 . \dot{0}$

舀

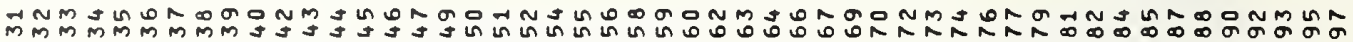

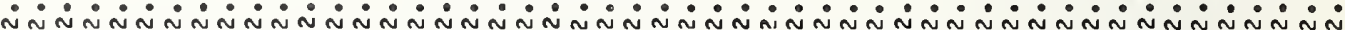

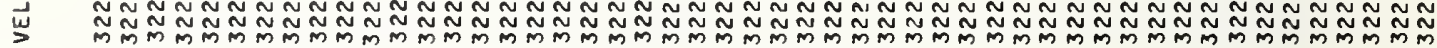

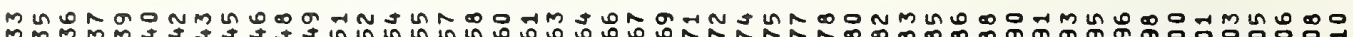

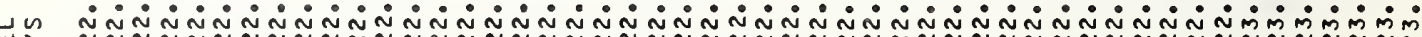

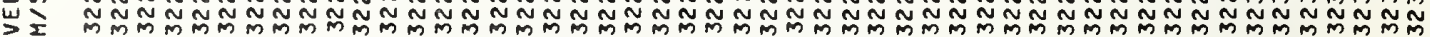

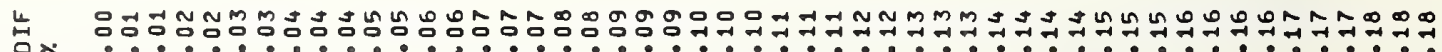

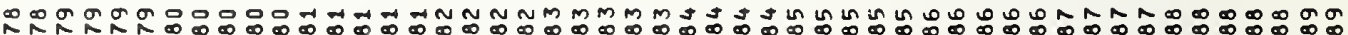

$\vec{z}$

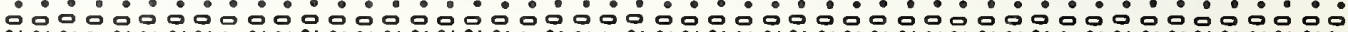

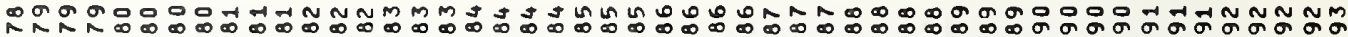

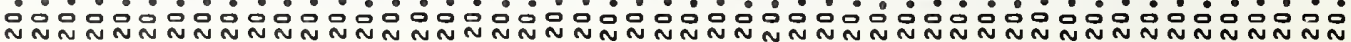

占

m

w

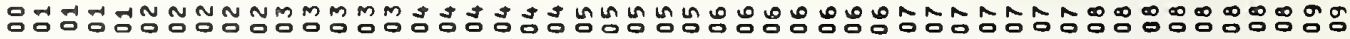
i i i i i i i i i i i i i i i i i i i i i i i i i i i i i i i i i

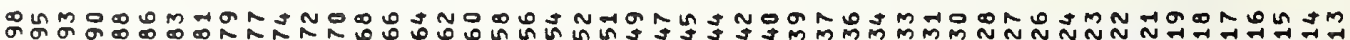
$\sum_{\text {岁 }}^{n}$

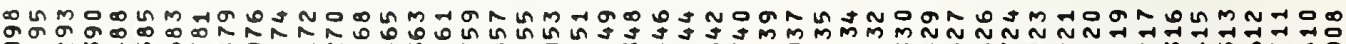

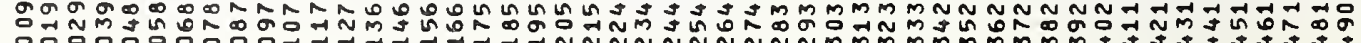

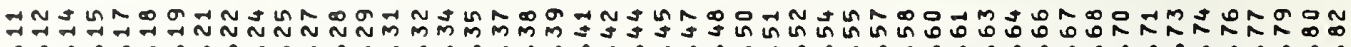

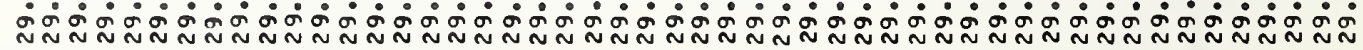

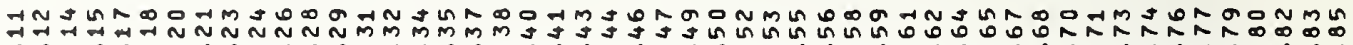

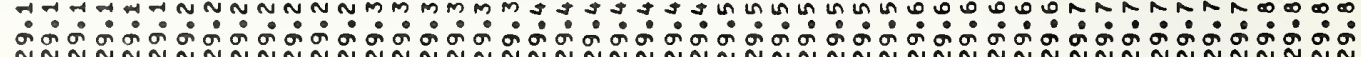

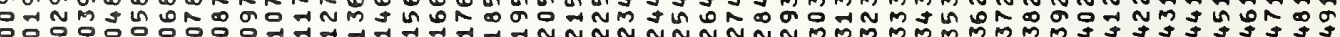

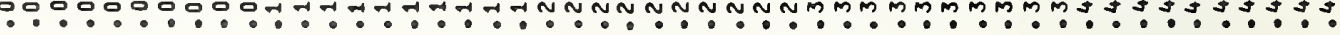

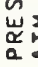

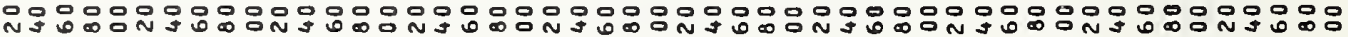

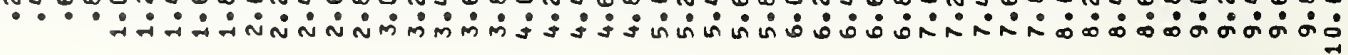




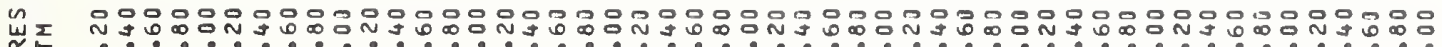
造 - -

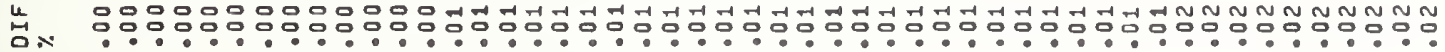

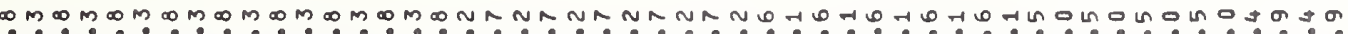

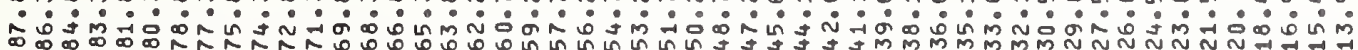

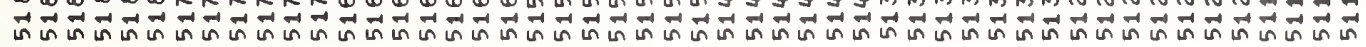

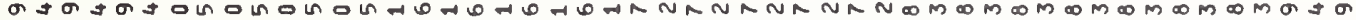

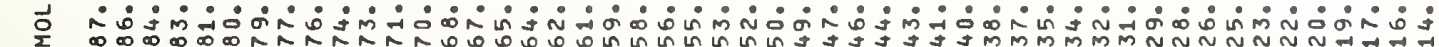

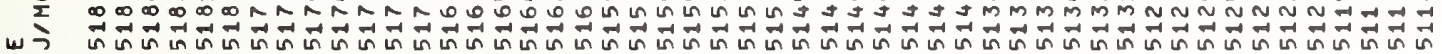

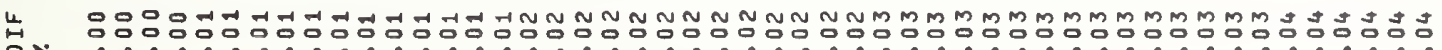

ㄴ?

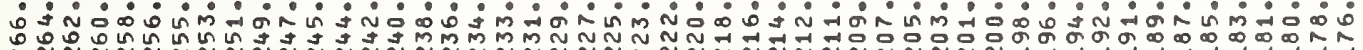

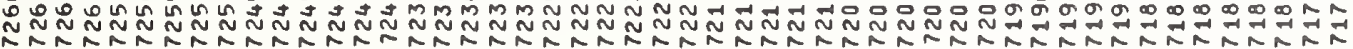

N 0 H HM

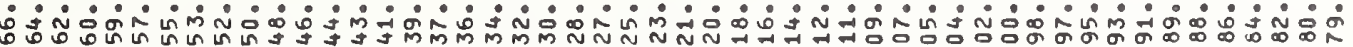

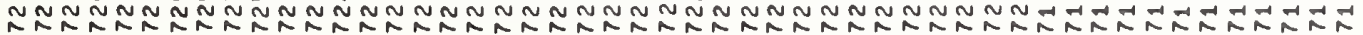

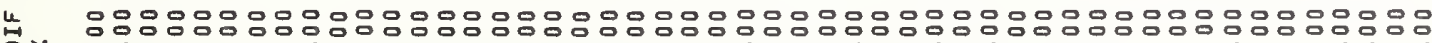

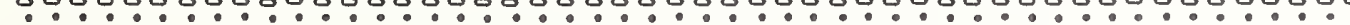
ำด бুmó:

$\backsim$

울

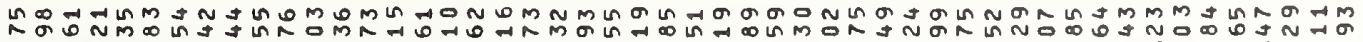

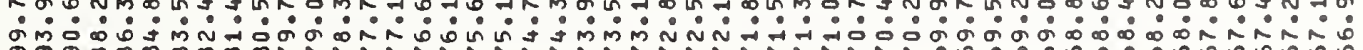
$\operatorname{lom}_{\rightarrow \rightarrow \infty}^{\infty} \log _{-1}^{\infty}$

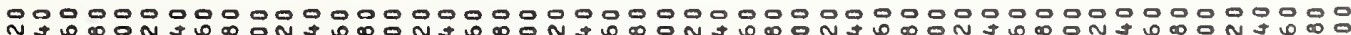
- 


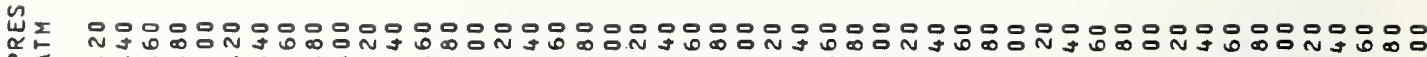

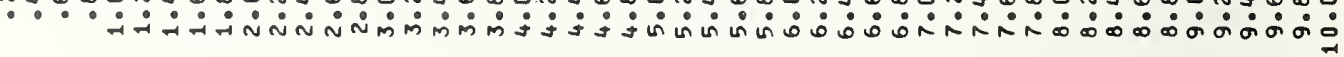

L

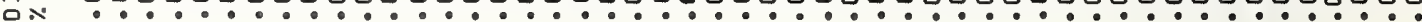

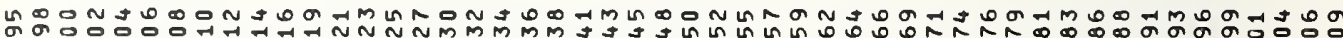

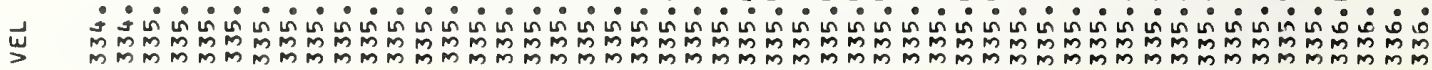

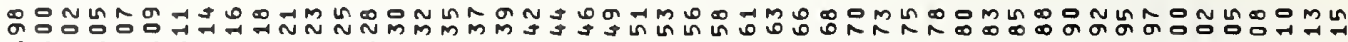

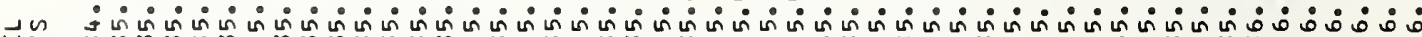

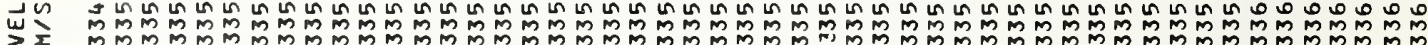

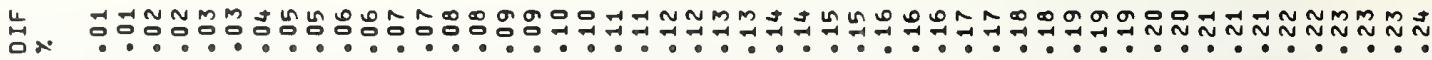

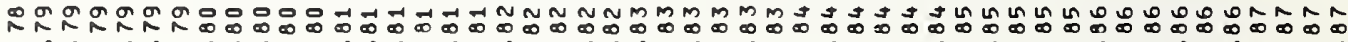

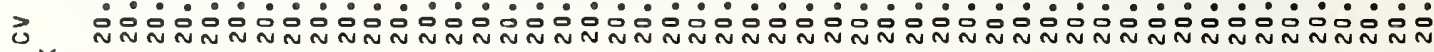

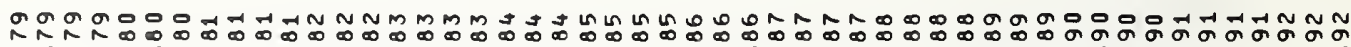
u

出

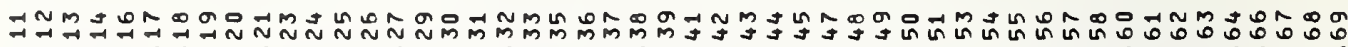
i

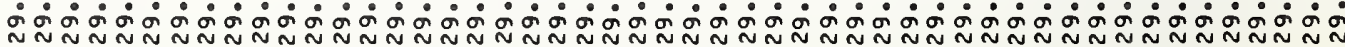

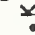

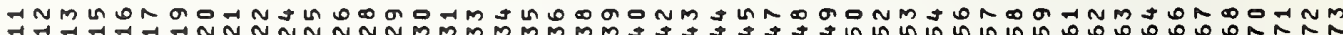

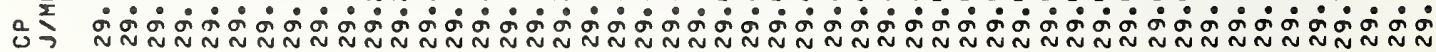

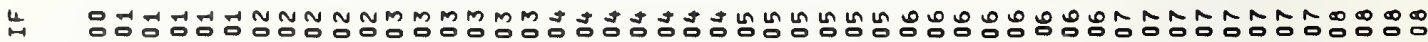

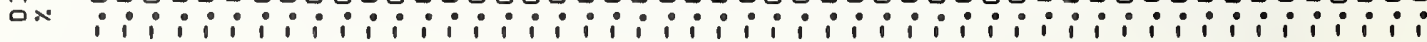

虽 z

แ Z东 


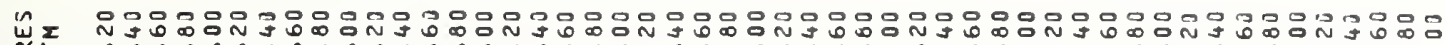
然

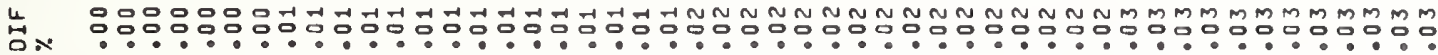

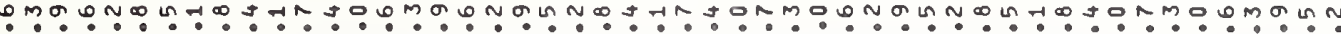

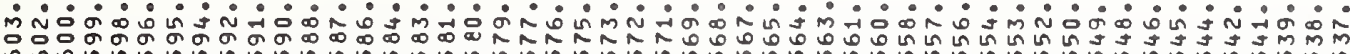

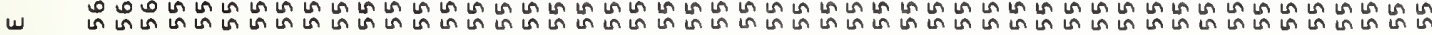

^MONT-1

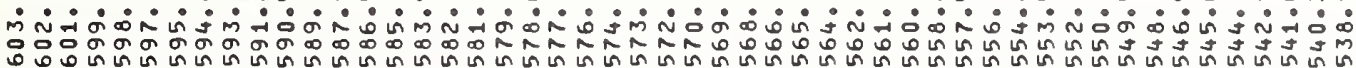

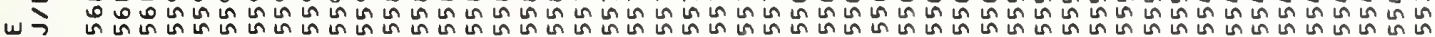

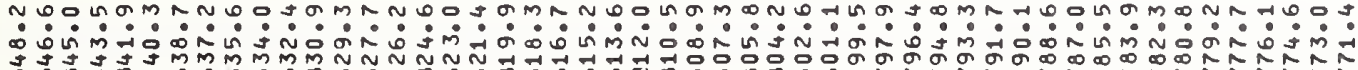

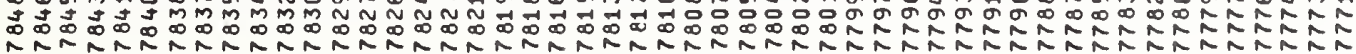

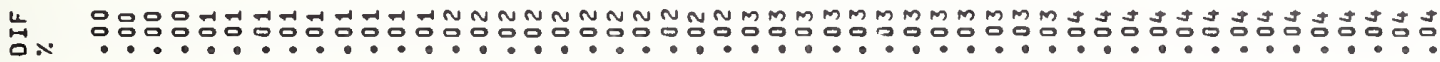

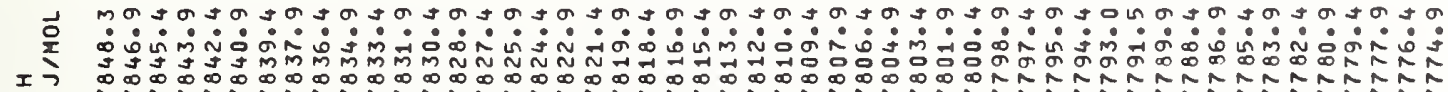

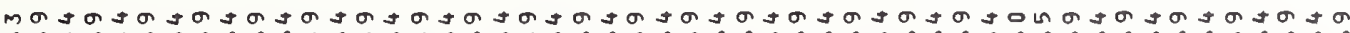

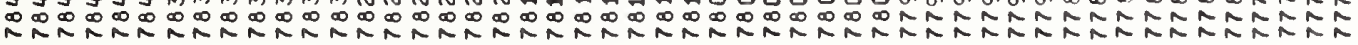

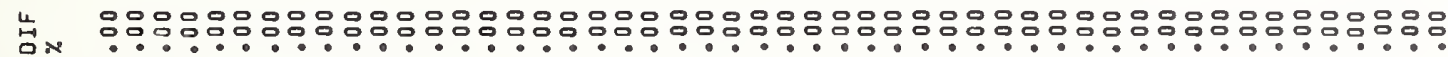

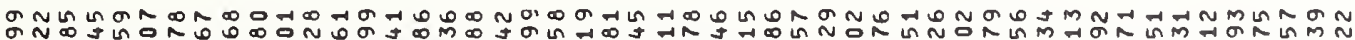

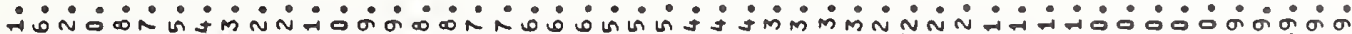

n 운

崖道

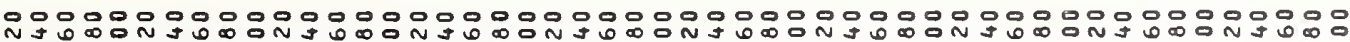

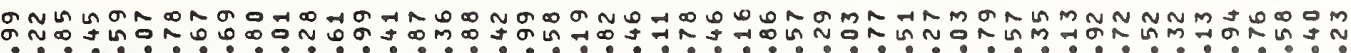

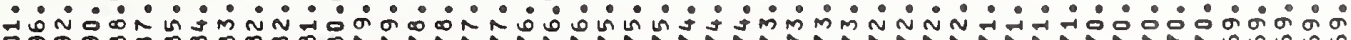

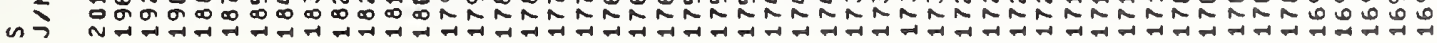

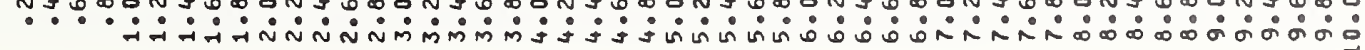




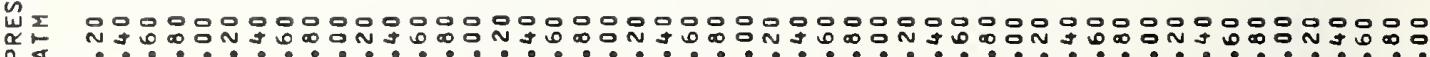

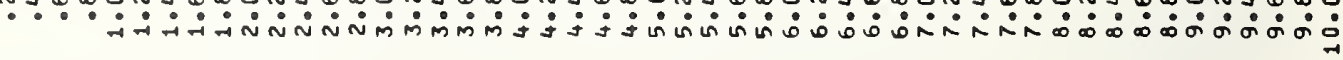

台我 Mㅁำ

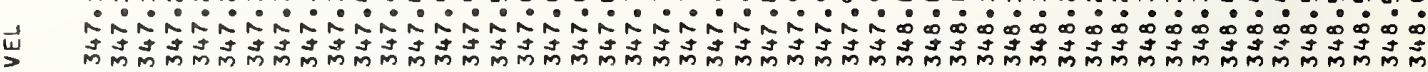
茾西

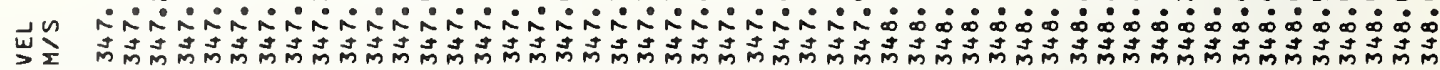

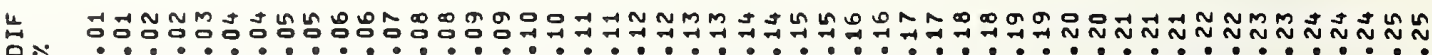

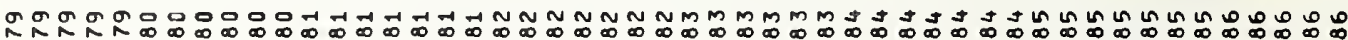

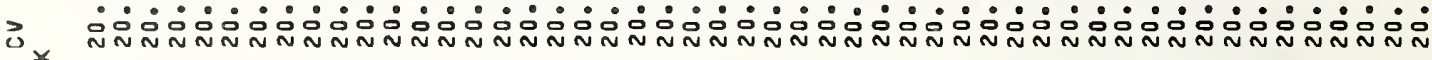

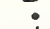

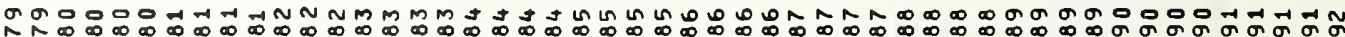

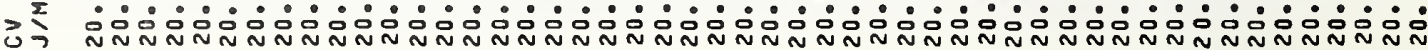
台

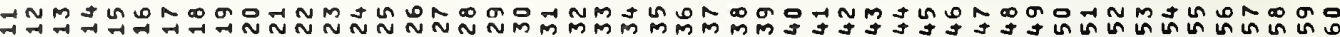

옹 צ.

운

¿i

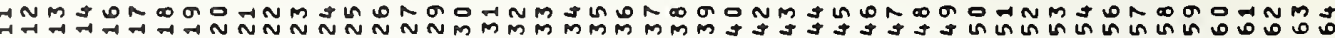

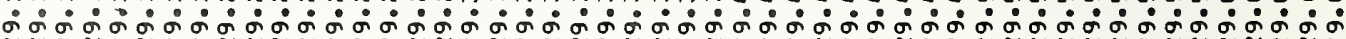

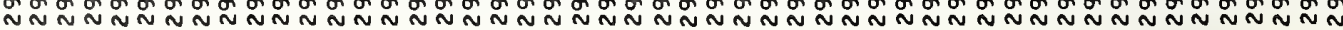

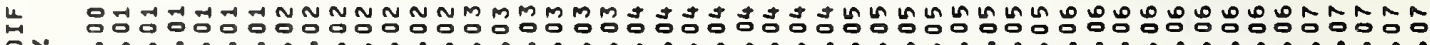

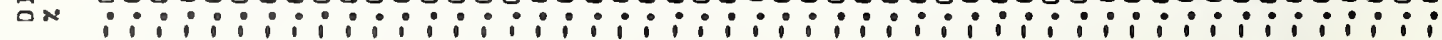

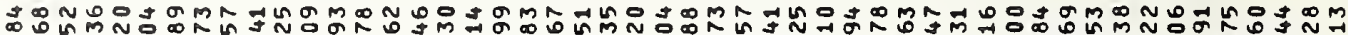

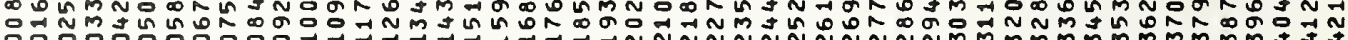

㟧

出훙

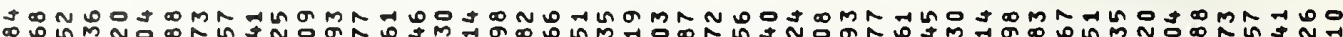

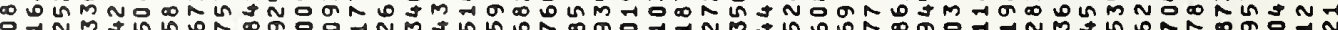
$\because \because 0: 0$

$\stackrel{\substack{w \\ \alpha}}{a}$

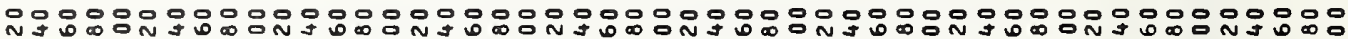

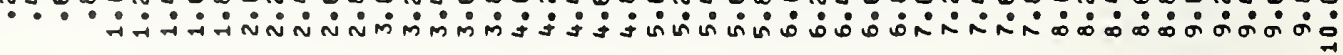




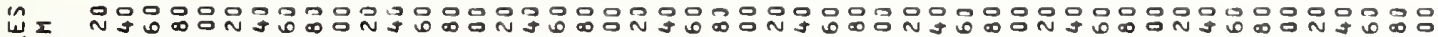
造

台

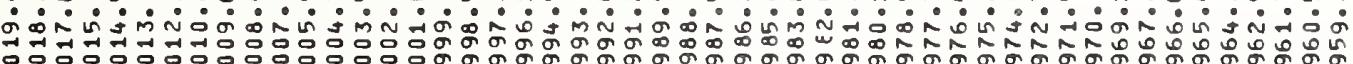

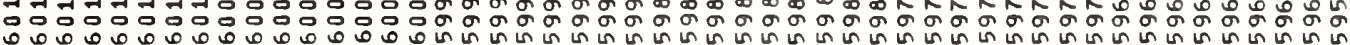

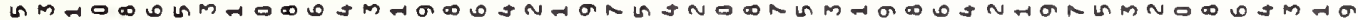

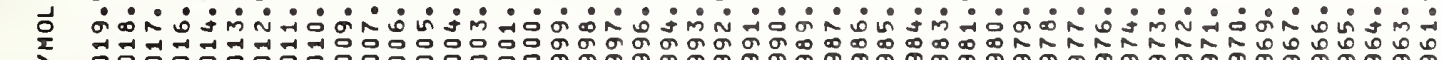
ए

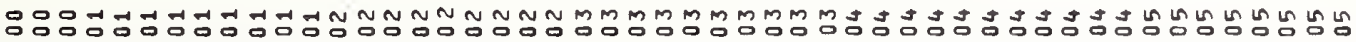
․ำ

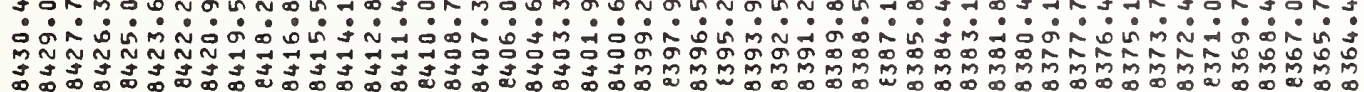

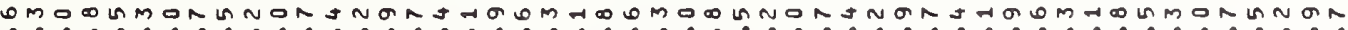

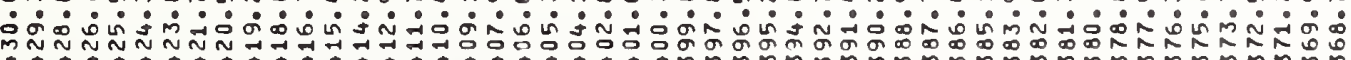

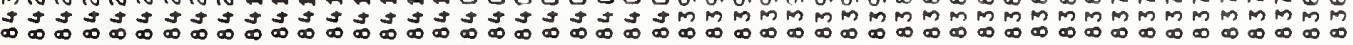

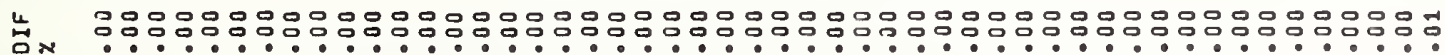
ลี

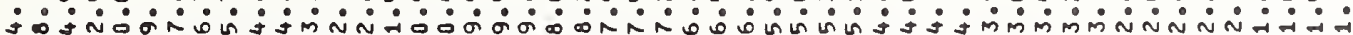

is

צ

일

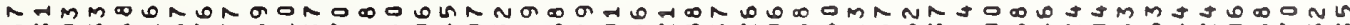
ำ

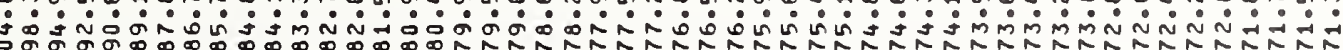
ก

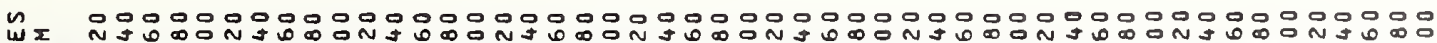

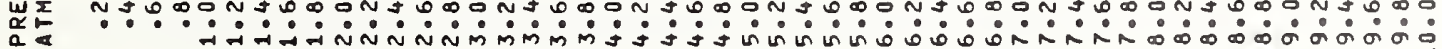




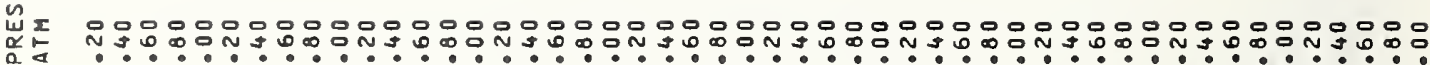

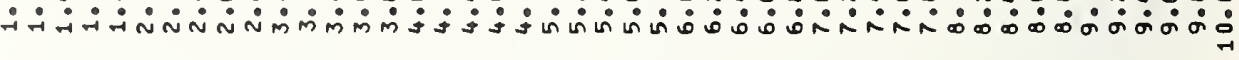

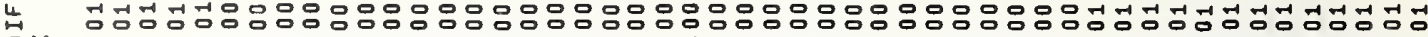

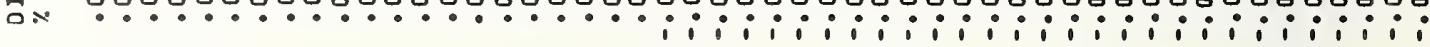

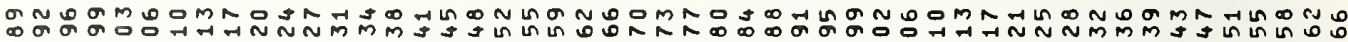

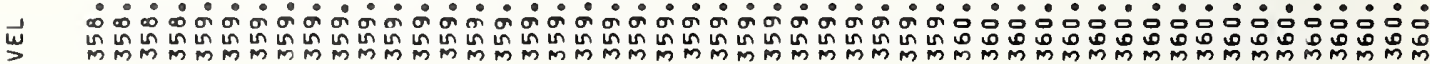

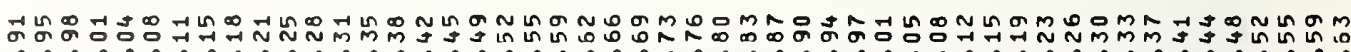

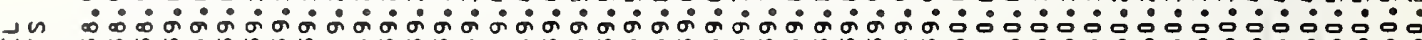

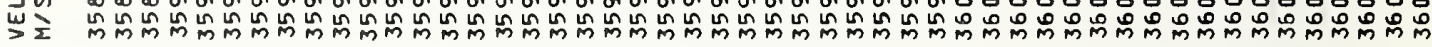

แ

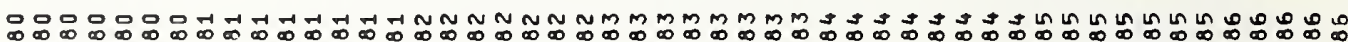

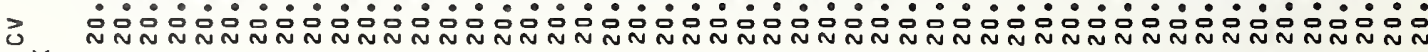
$x$ 员品

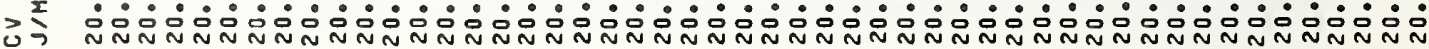

แ

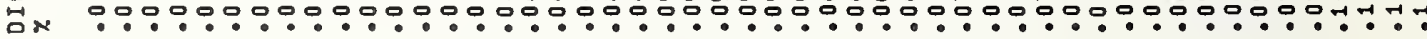

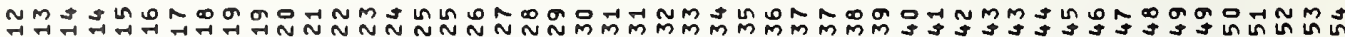
옹

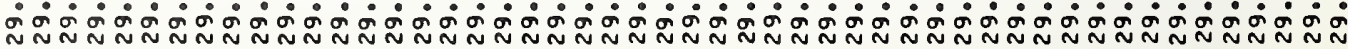

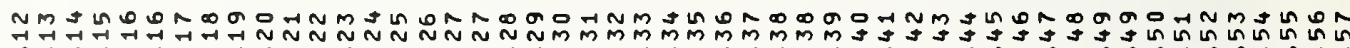

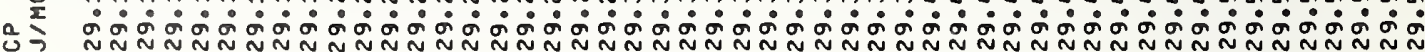

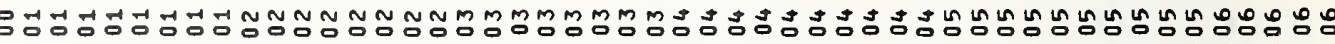
ore $i$ i i i i i i i i i i i i i i i i i i i i i i i i i i i i i i i i i i i

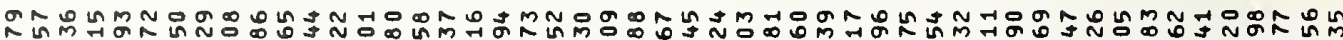
w

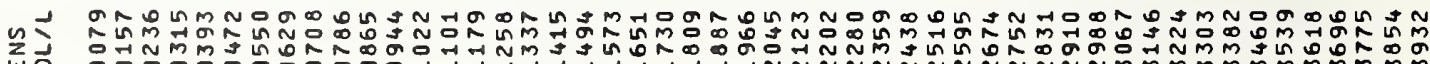
wo

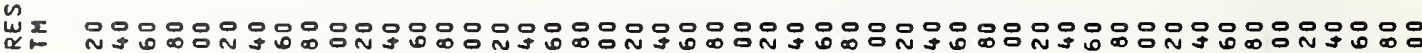
a $\because .90$ 


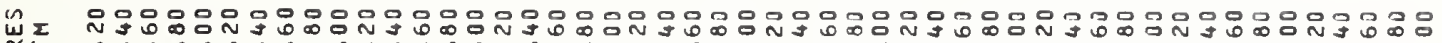
造

背

MNFOGN

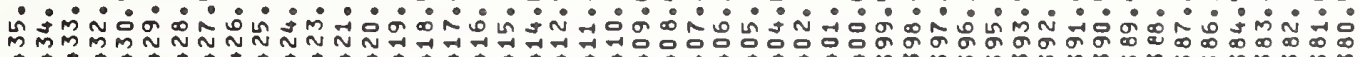

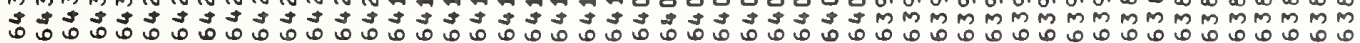

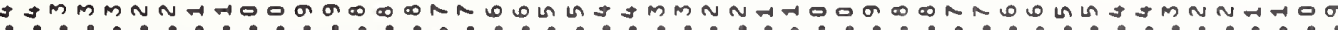

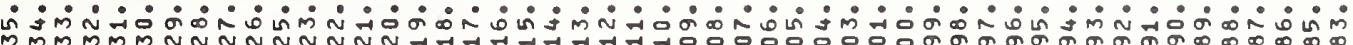

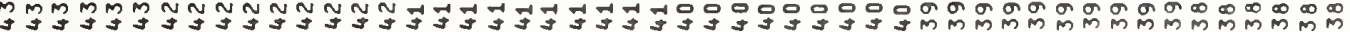

w

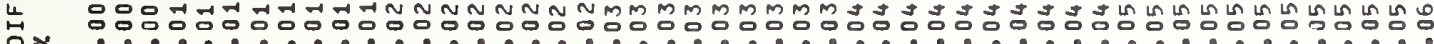

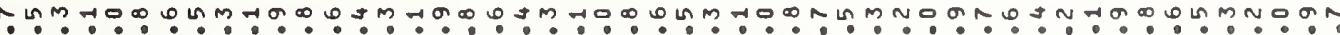

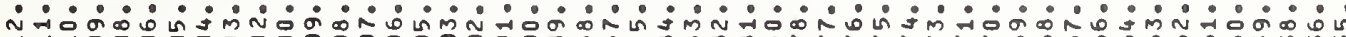

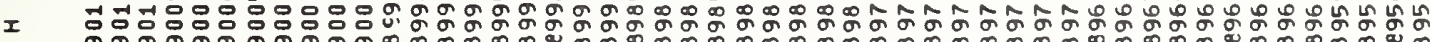

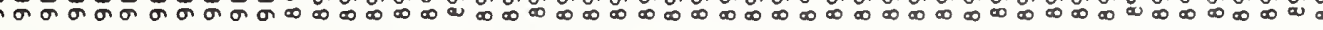

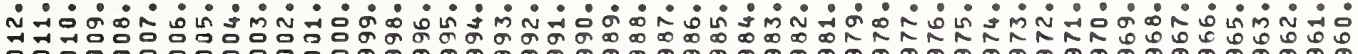

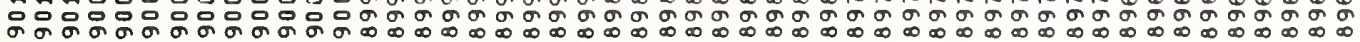

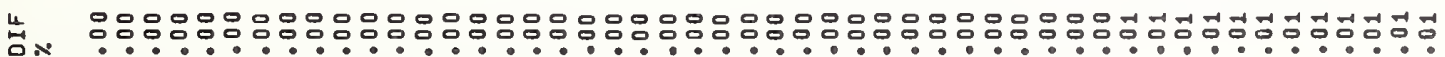
ปี

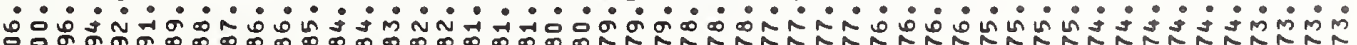

us .

훌

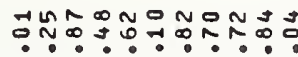

OM

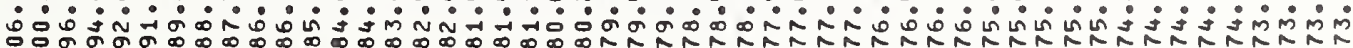

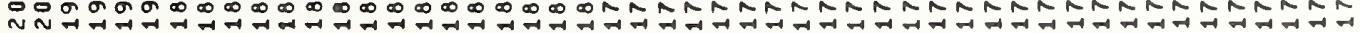

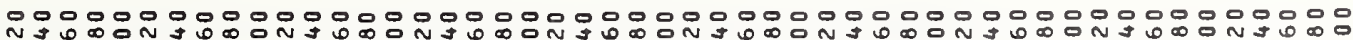
: 


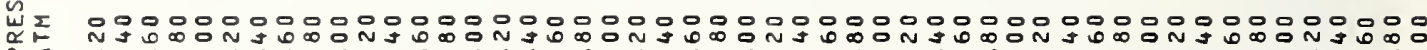
a $\because \because \cdots$

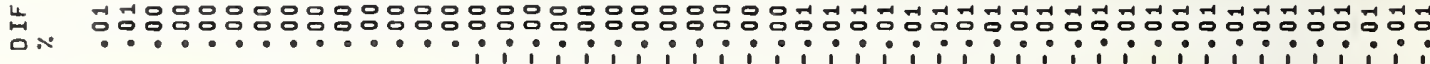

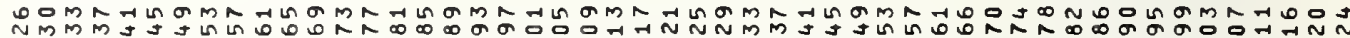

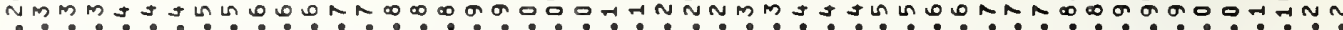

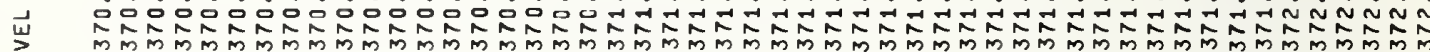
DN⿴囗十心

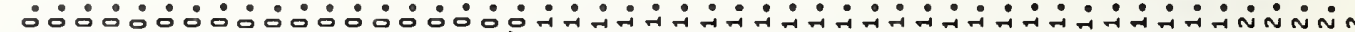

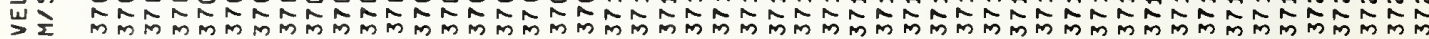

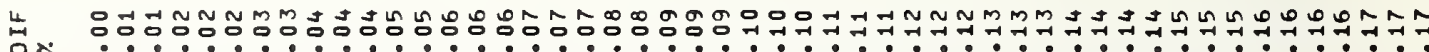

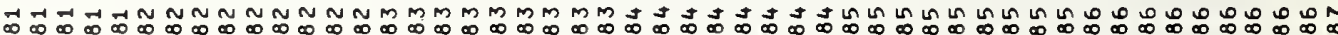

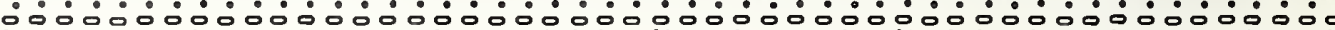
3 N

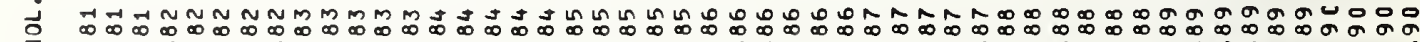
份

崖 m

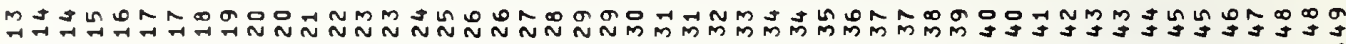

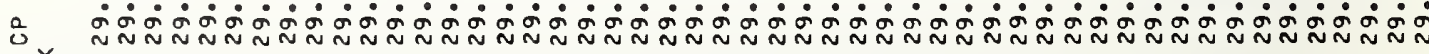

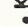

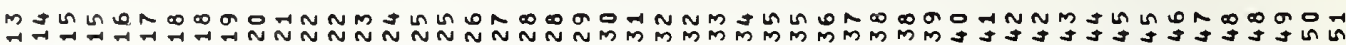

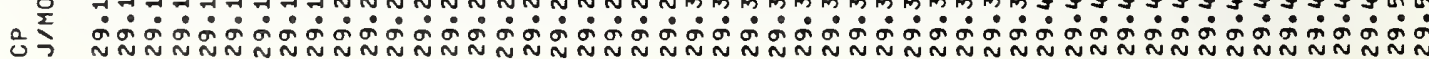

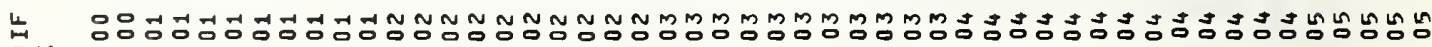

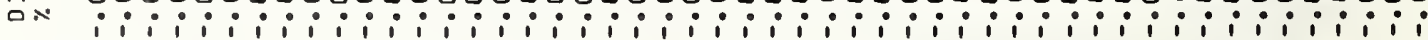

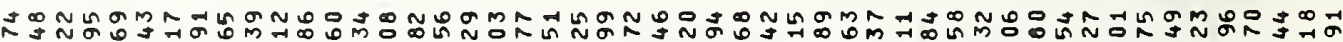

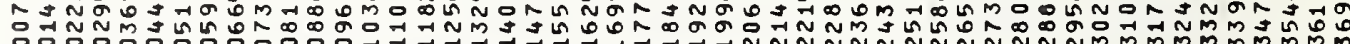
㟧 I 0 N

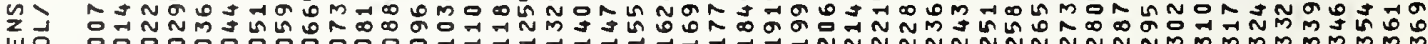

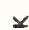

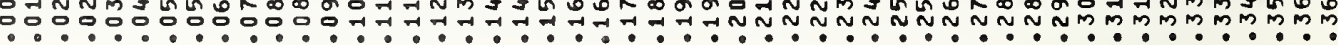

崖

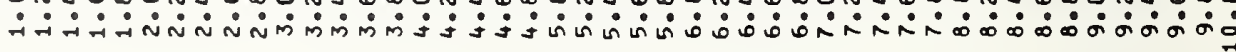




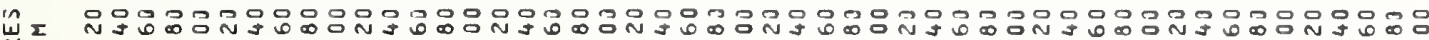

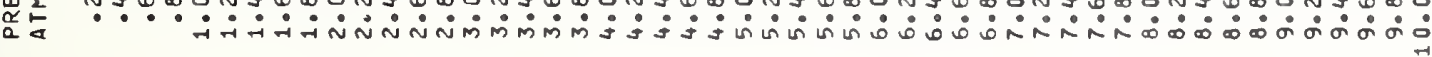

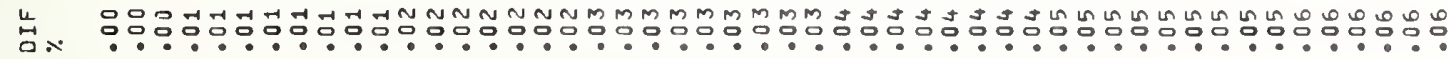

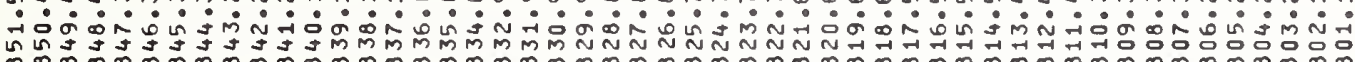

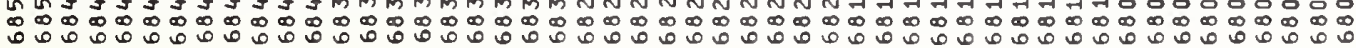

$00 N \infty \infty 000$ H

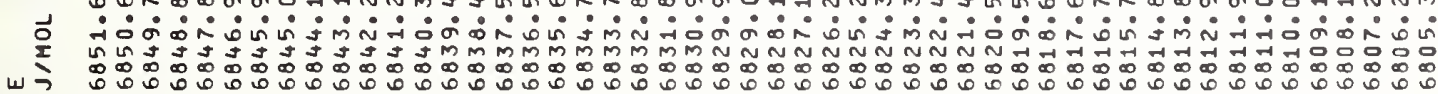

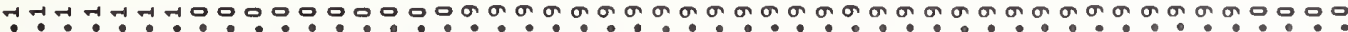

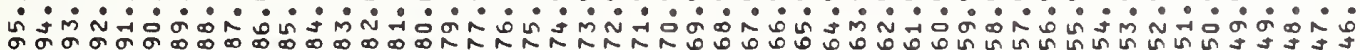

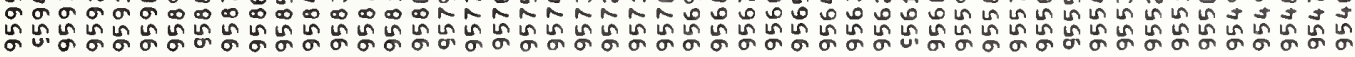

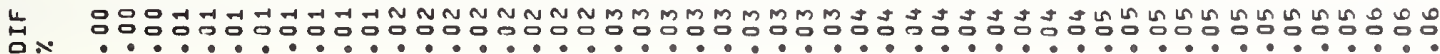

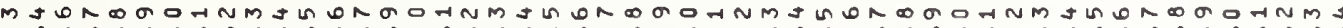

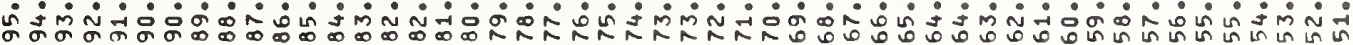

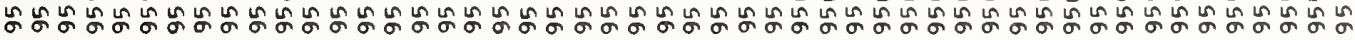

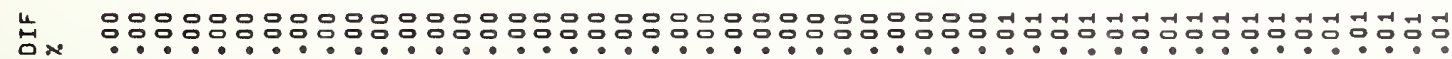

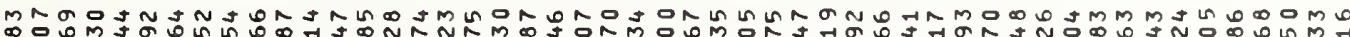

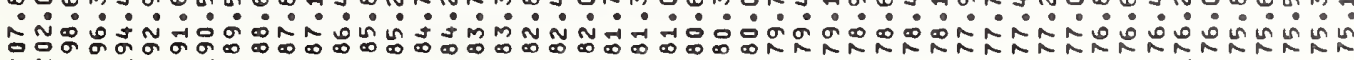

u

$$
\text { ì }
$$

\section{ind}

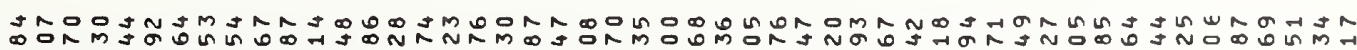

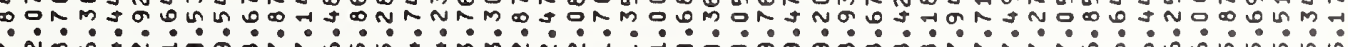

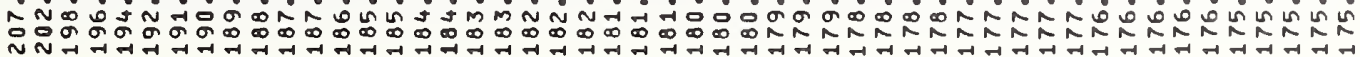

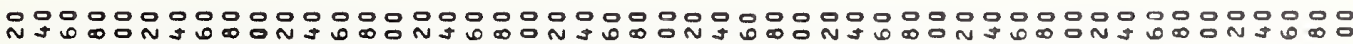

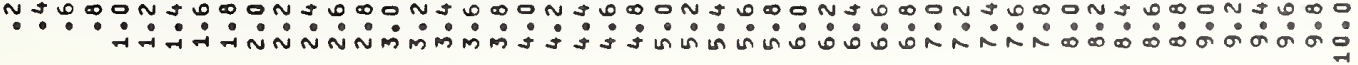




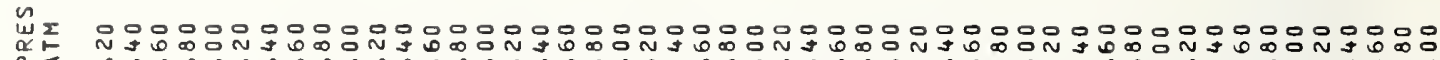

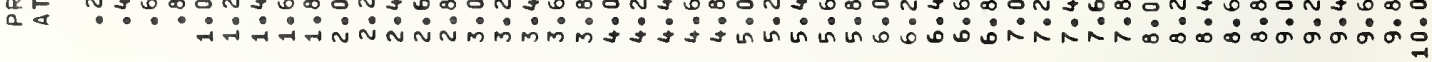

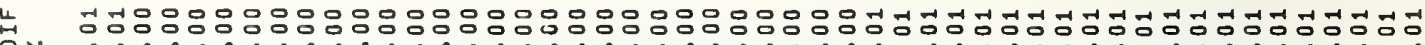

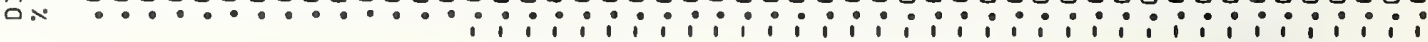

ํㅠำ

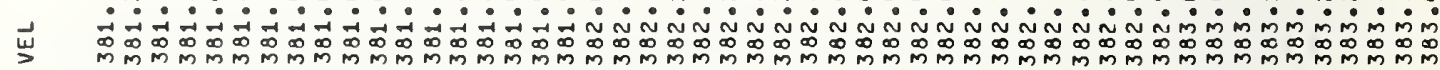

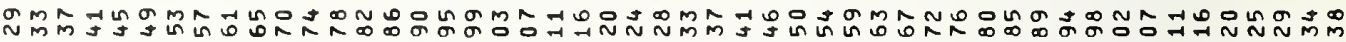
\lrcorner u

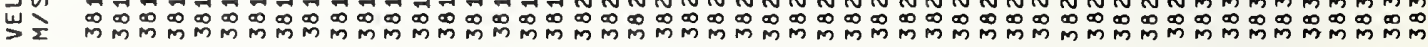

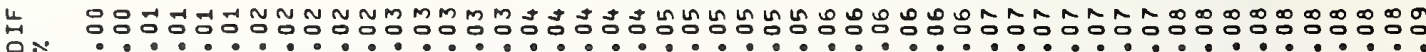

M M M M M M M M M $M$

$\overrightarrow{0}$

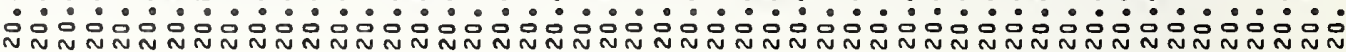

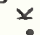

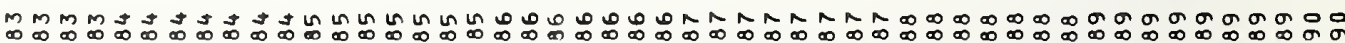

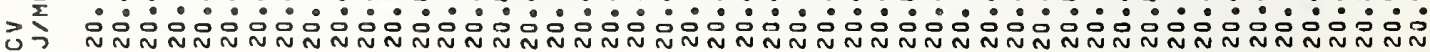

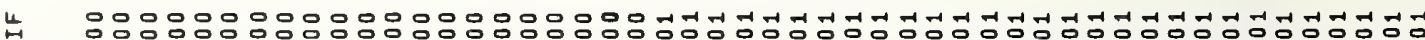

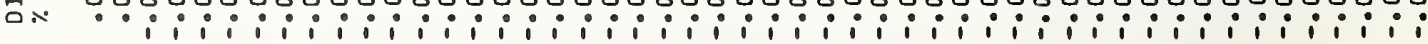

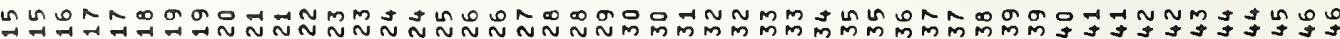
온

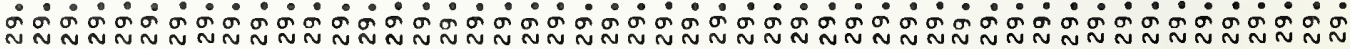

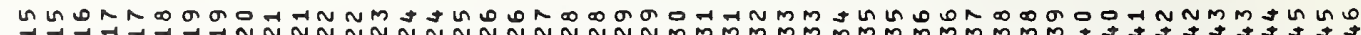

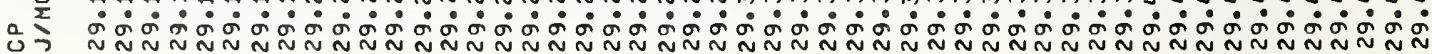

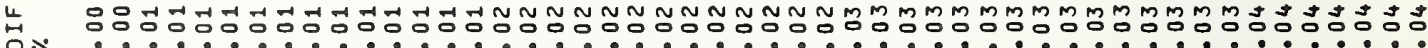

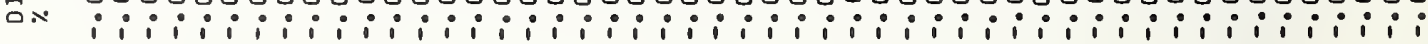

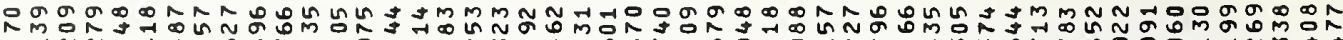

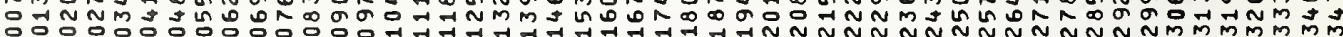

岁

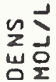

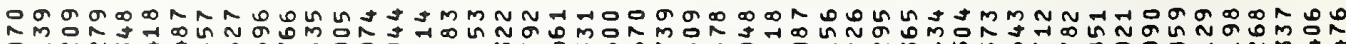

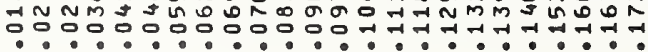




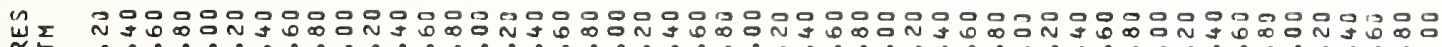
Q

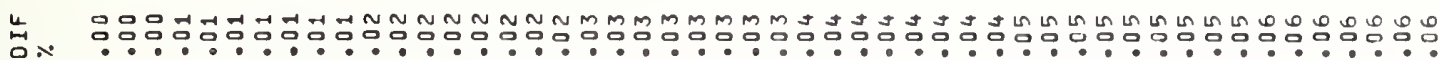

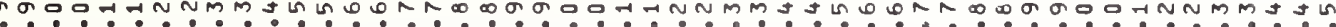

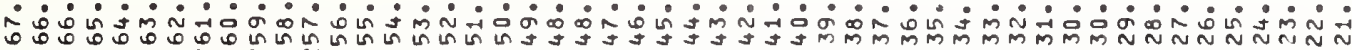

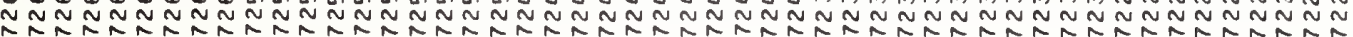

- HM

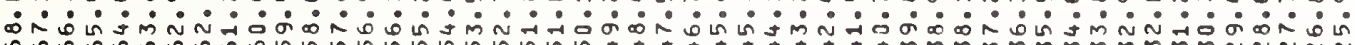

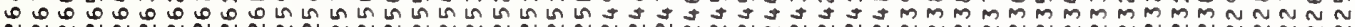

W

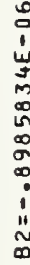

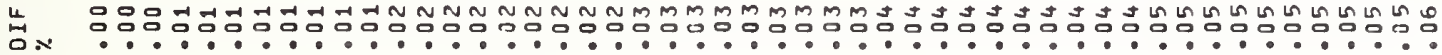

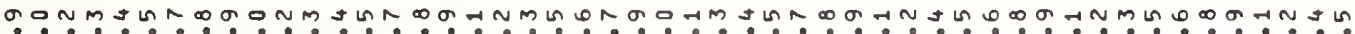

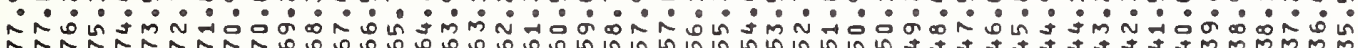

I

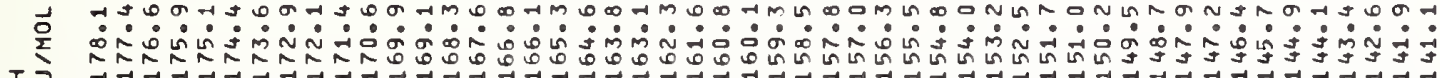

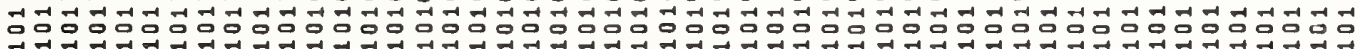

出

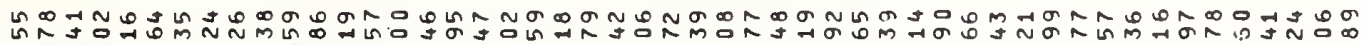

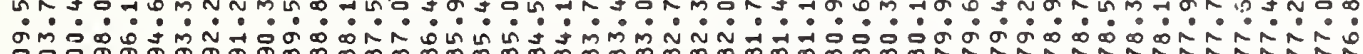

a

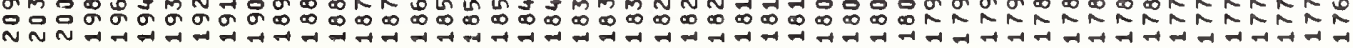

n क्र いう N

出

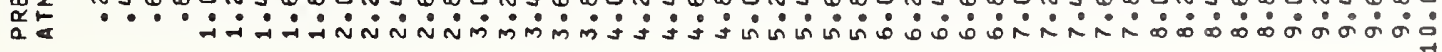




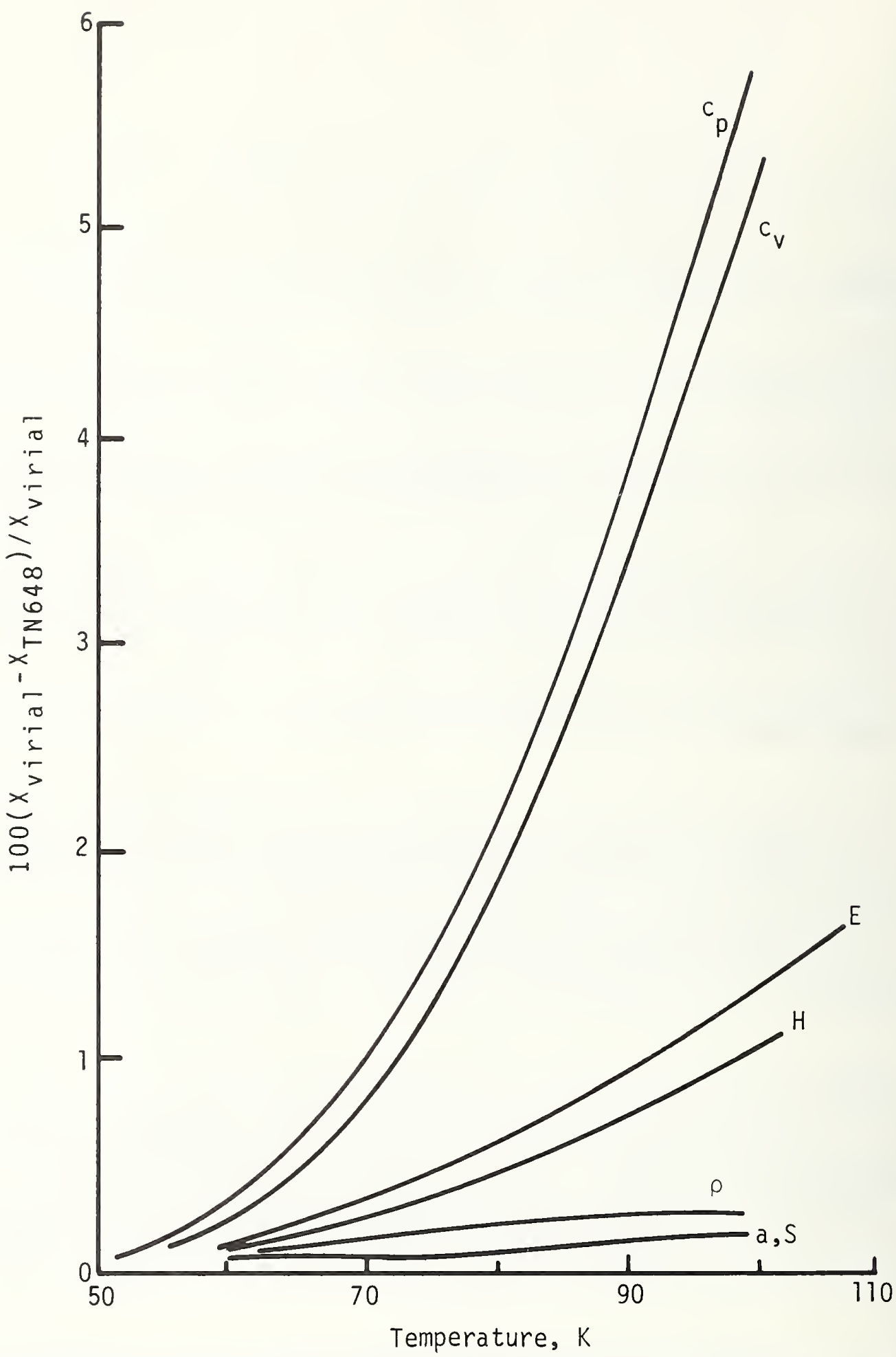

Figure 1.- Percent of deviation from TN 648 values at the saturation boundary for various thermodynamic properties $\mathrm{x}$. 


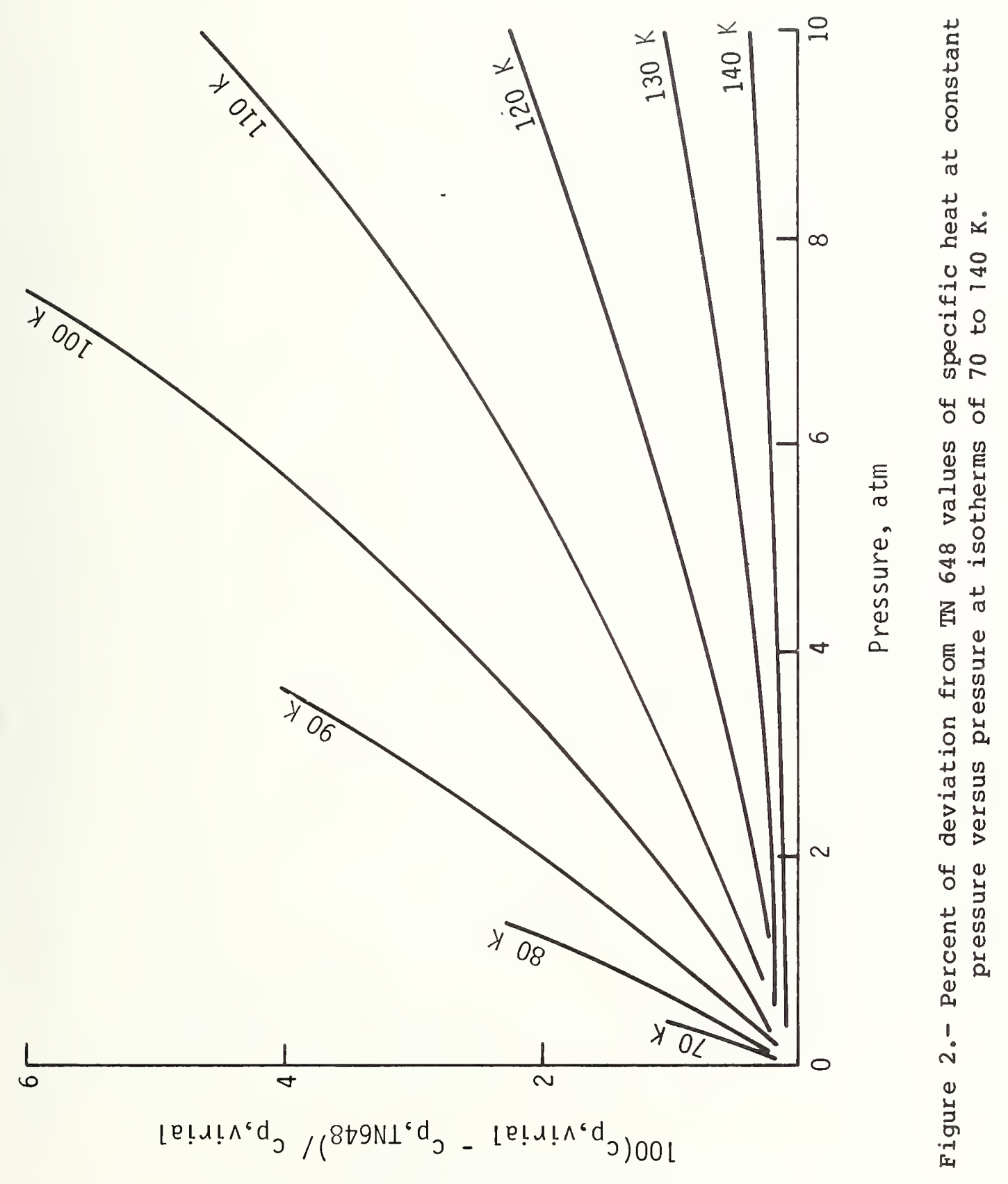




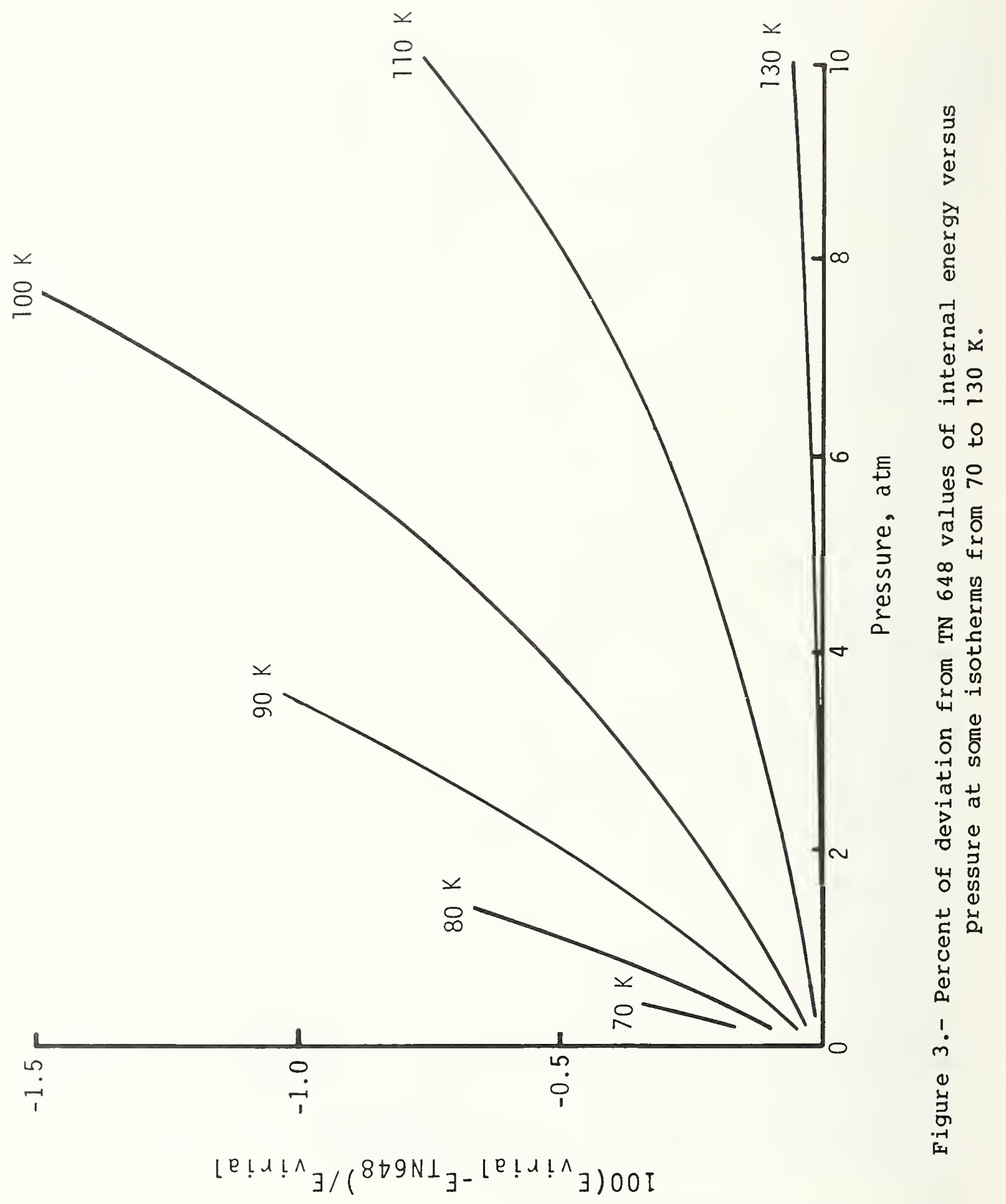




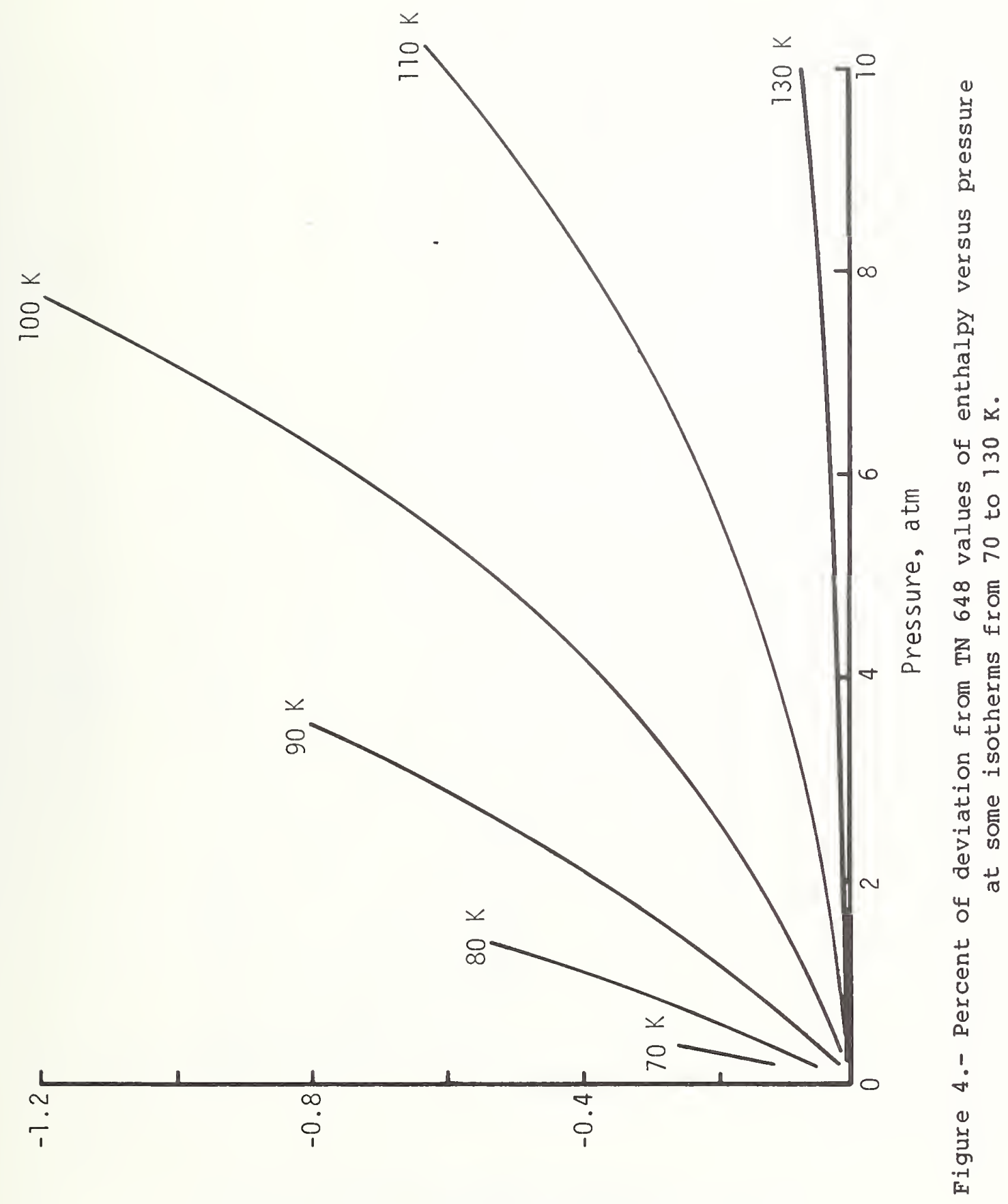

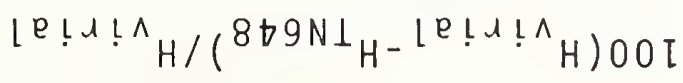




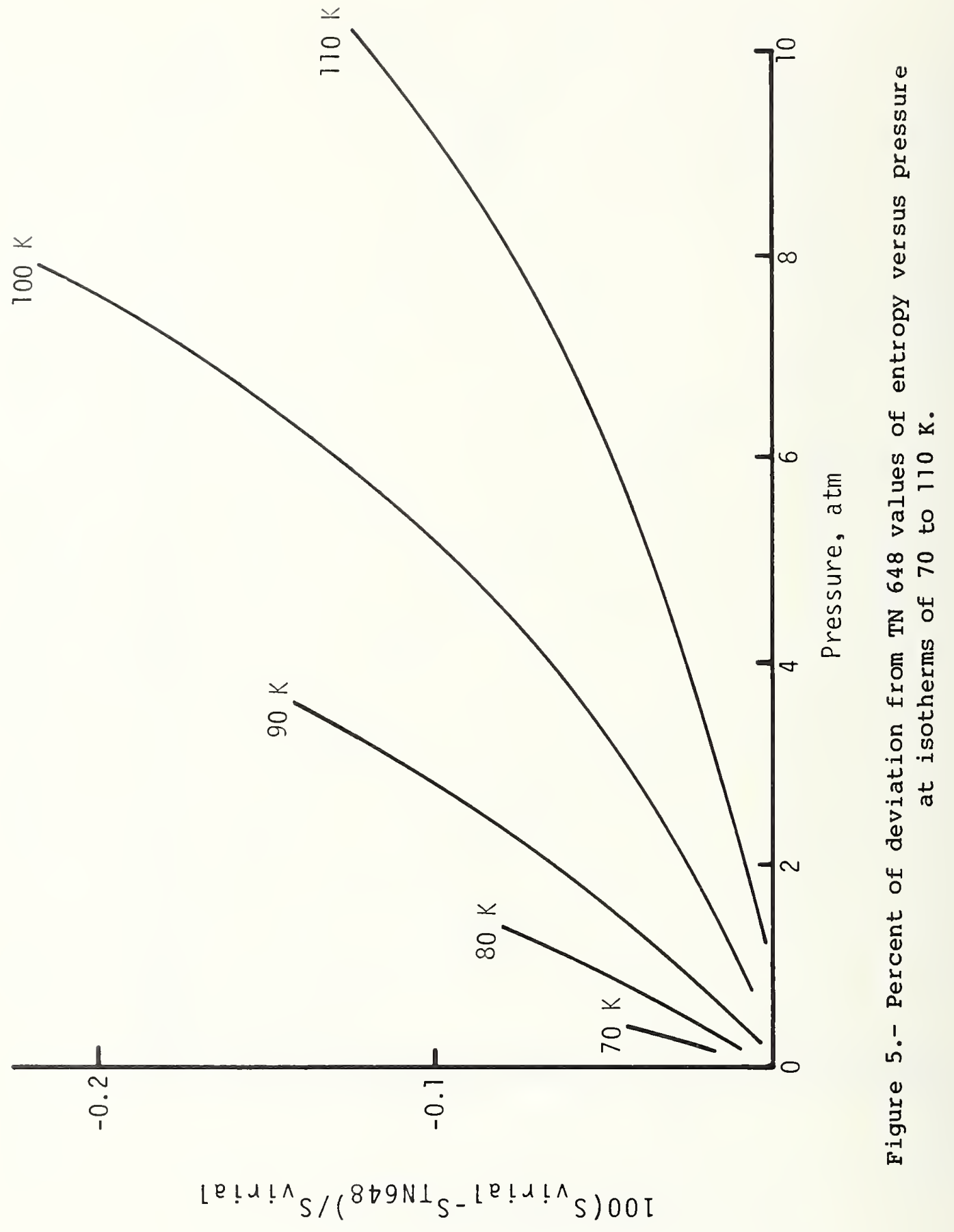




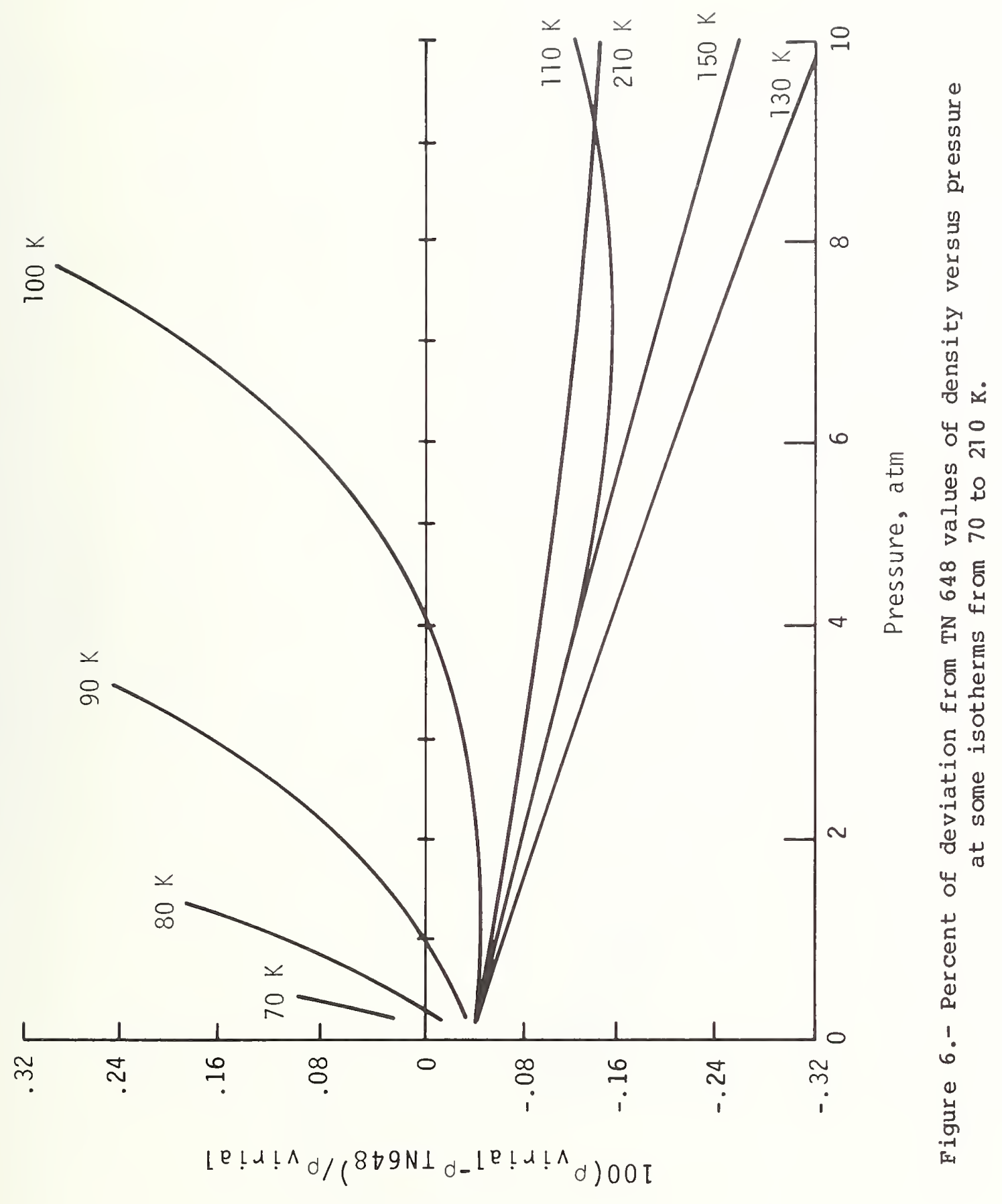




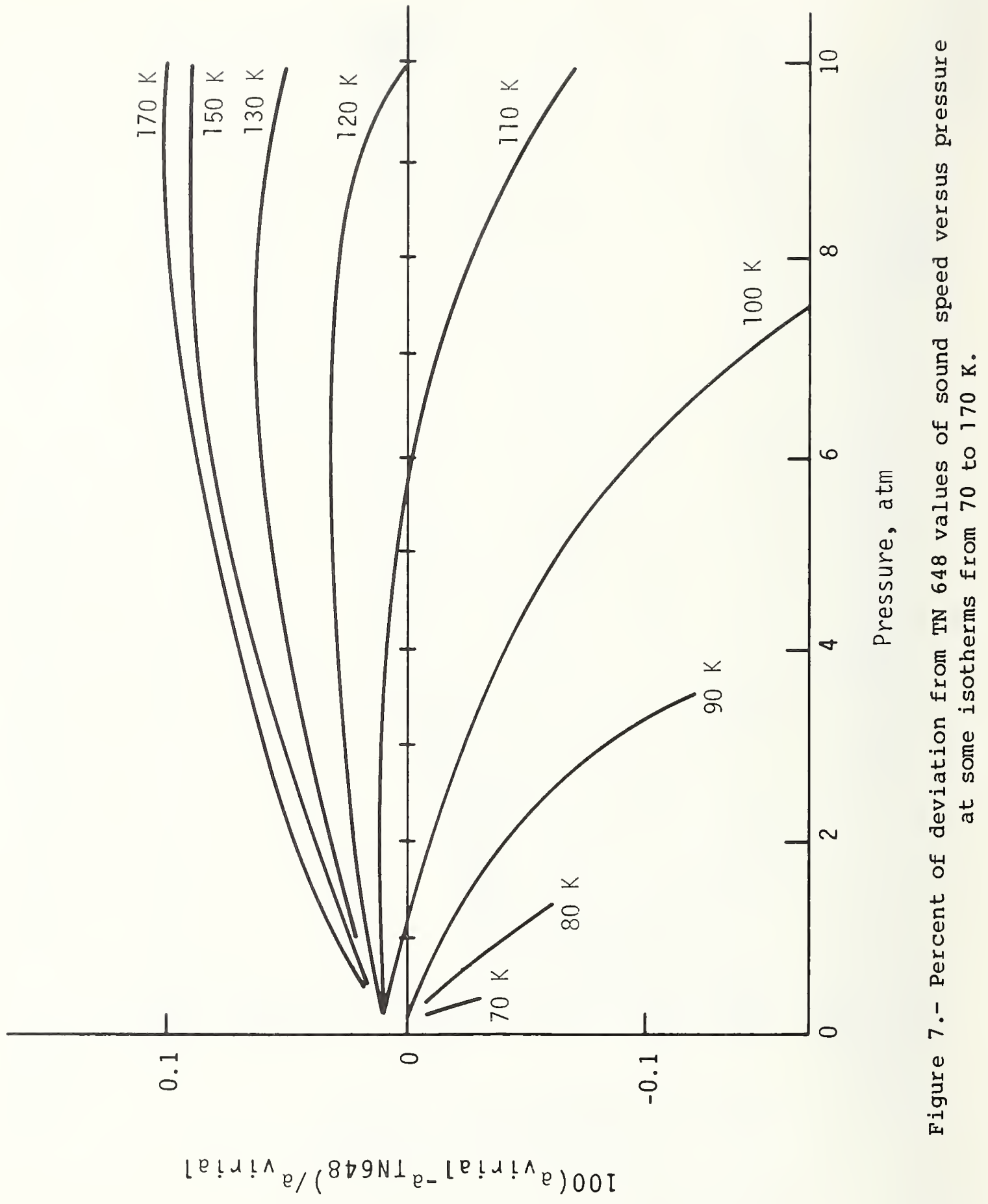



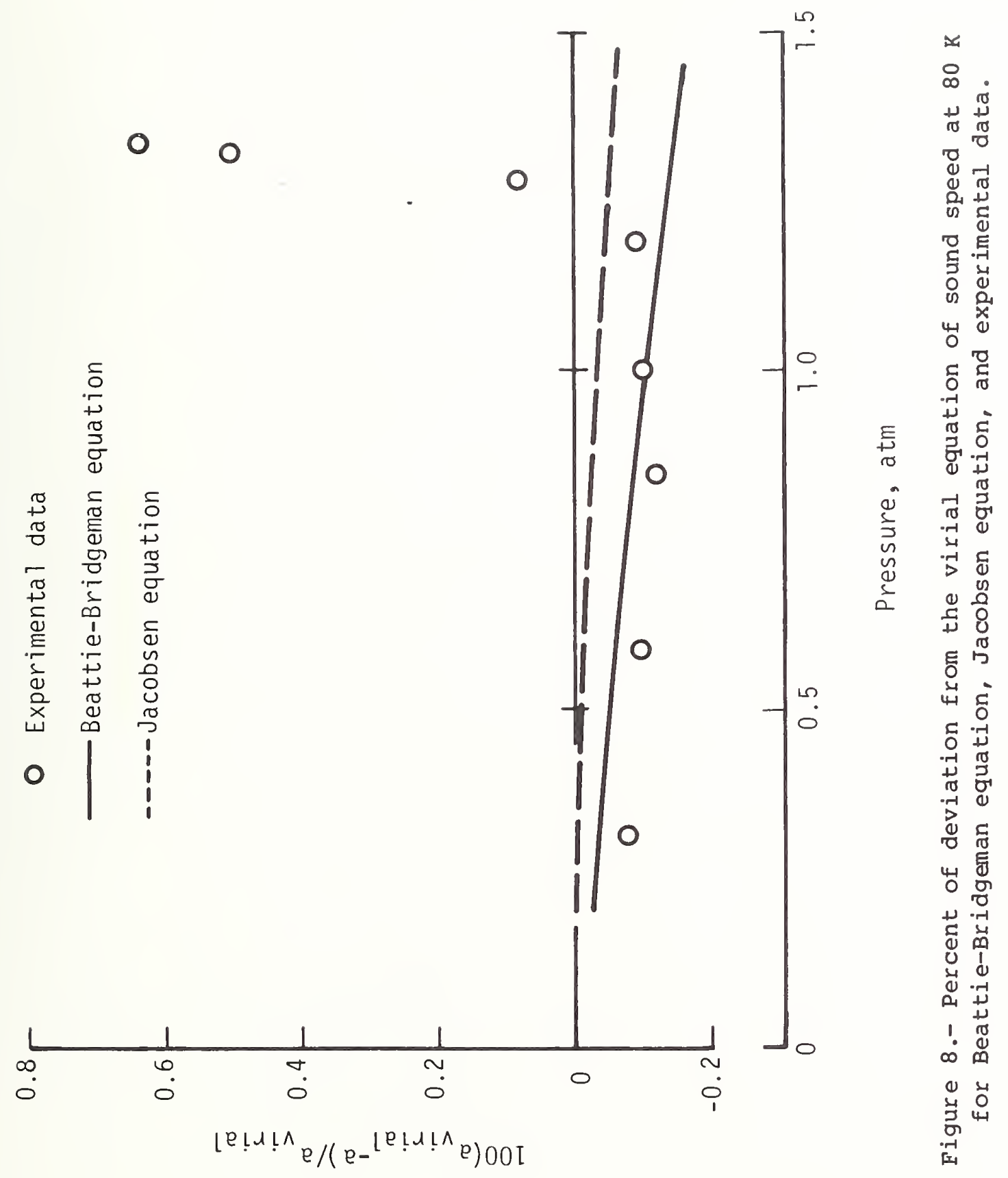


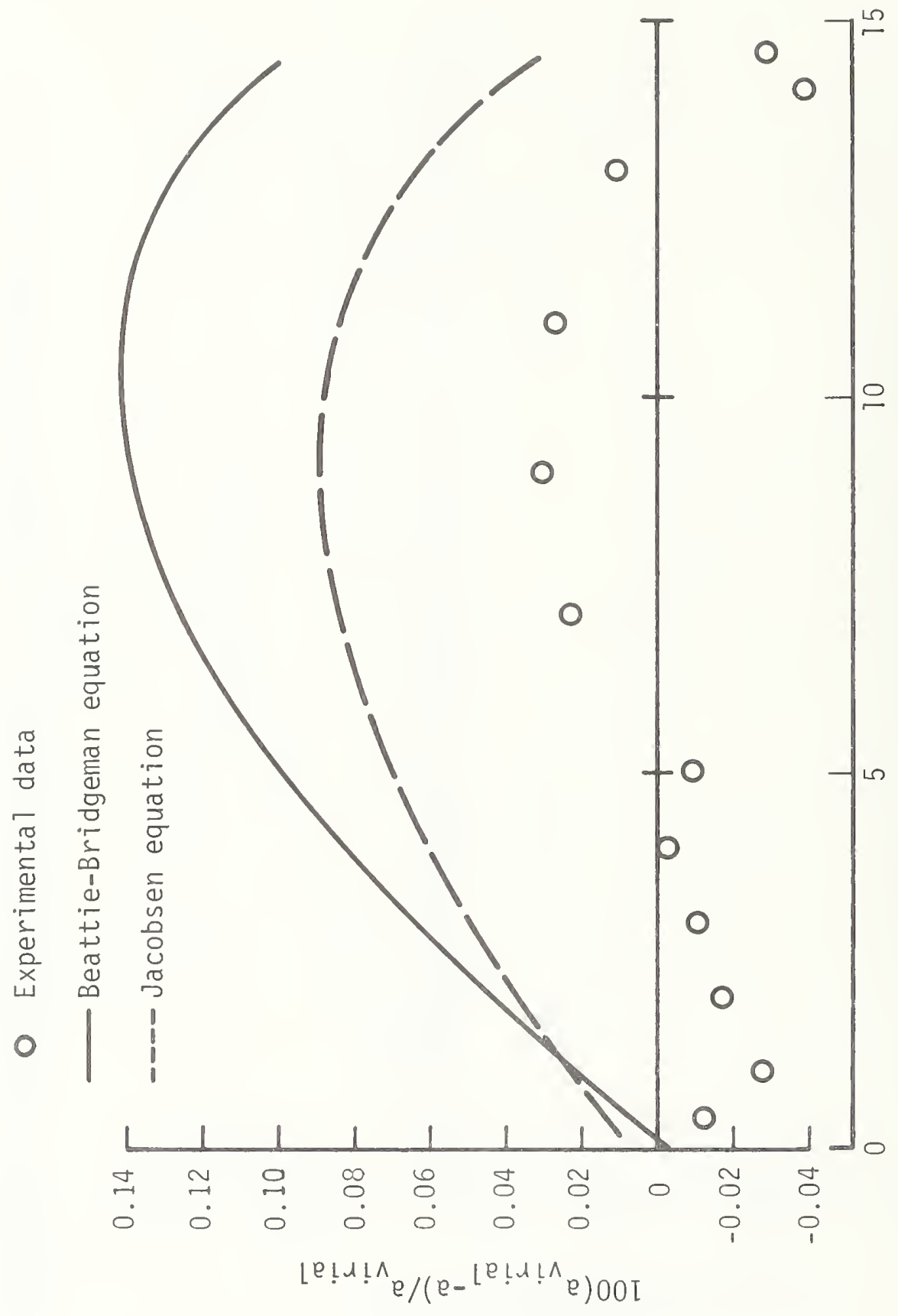

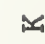

은 崩

r

ఖ

少

吕

马

ט

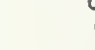

E

$0 \pm$

仓

乡

जิ

c

$\stackrel{-1}{>}$

용

ธี

出

동

岱

व

出 导

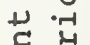

ठิ

- 1

品

10

aे

䓀

品 


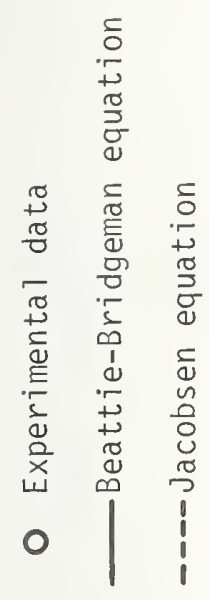

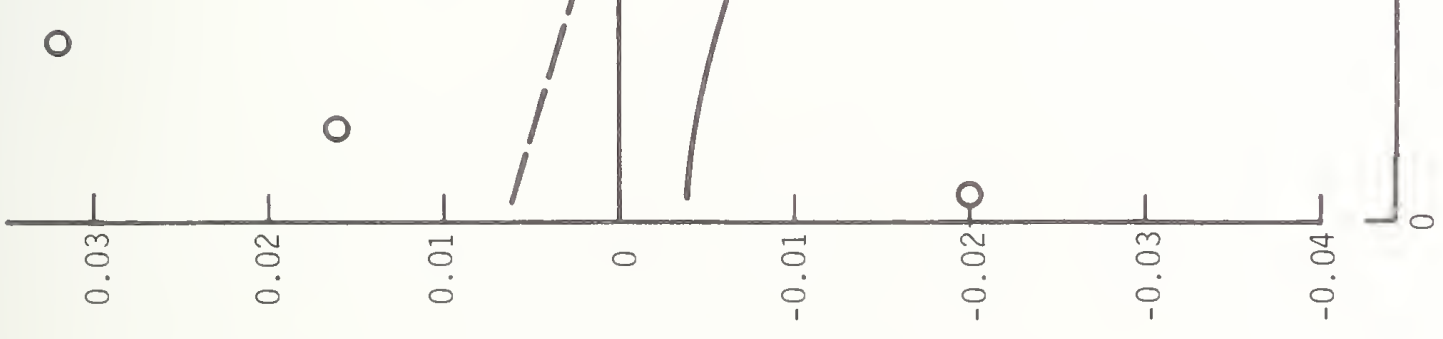

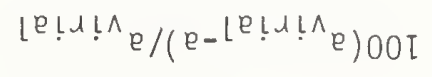

ᄃ 


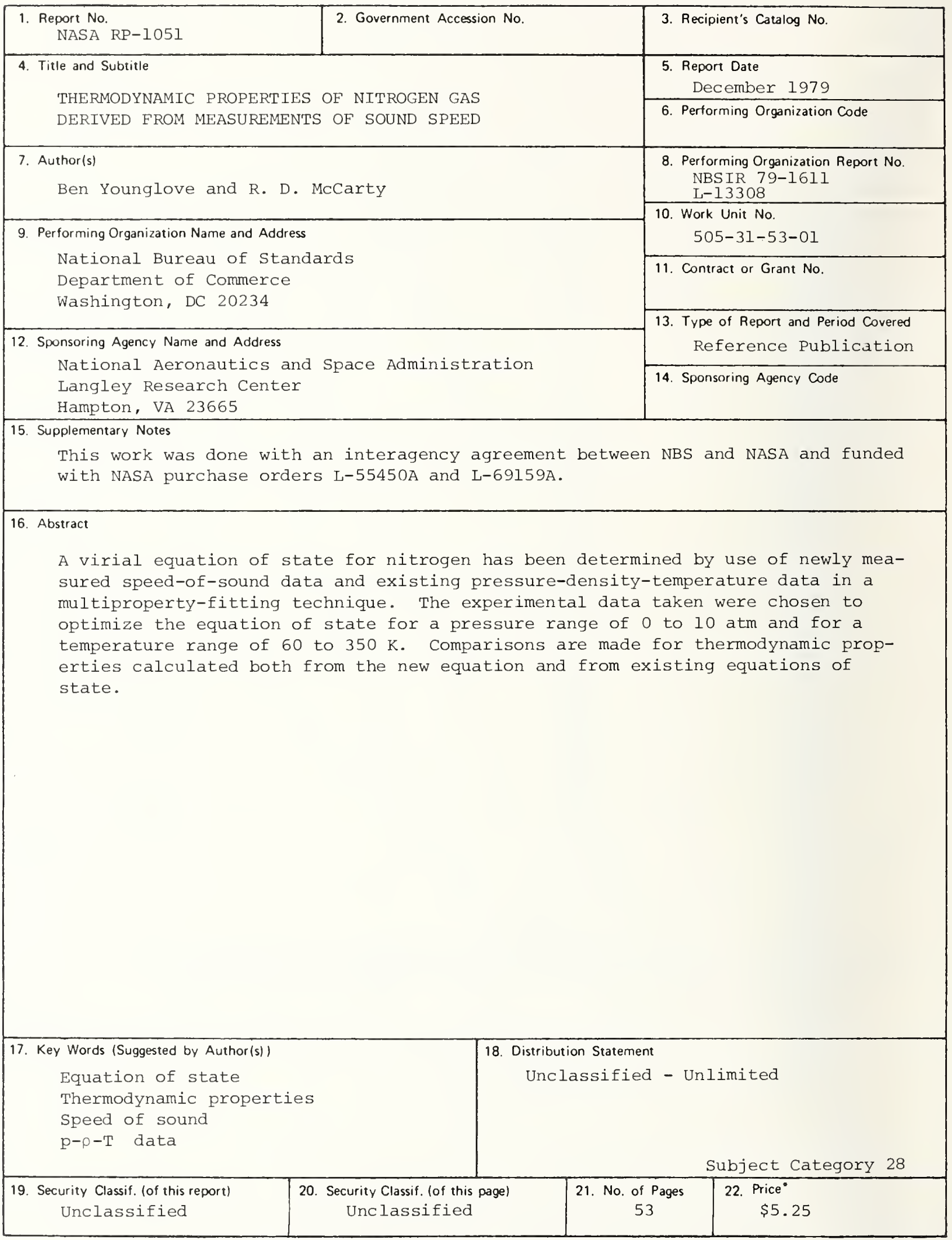




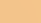


Space Administration

Space Administration

Washington, D.C.

NASA-451

20546

Official Business

Penalty for Private Use, $\$ 300$ 